\title{
Resource Allocation Strategies in Cognitive Radio D2D Communication NeTwORKS
}

\author{
by \\ Ajmery Sultana \\ Master of Science, University of Dhaka, 2008 \\ Bachelor of Science, University of Dhaka, 2006 \\ A dissertation \\ presented to Ryerson University \\ in partial fulfillment of the \\ requirements for the degree of \\ Doctor of Philosophy \\ in the Program of
}

Electrical and Computer Engineering

Toronto, Ontario, Canada, 2018

(C)Ajmery Sultana 2018 


\section{AUTHOR'S DECLARATION FOR ELECTRONIC SUBMISSION OF A DISSERTATION}

I hereby declare that I am the sole author of this dissertation. This is a true copy of the thesis, including any required final revisions, as accepted by my examiners.

I authorize Ryerson University to lend this dissertation to other institutions or individuals for the purpose of scholarly research.

I further authorize Ryerson University to reproduce this dissertation by photocopying or by other means, in total or in part, at the request of other institutions or individuals for the purpose of scholarly research.

I understand that my dissertation may be made electronically available to the public. 


\title{
Resource Allocation Strategies in Cognitive Radio D2D Communication Networks
}

Doctor of Philosophy 2018

Ajmery Sultana

Electrical and Computer Engineering

Ryerson University

\begin{abstract}
Device-to-device (D2D) communication is developed as a new paradigm to enhance network performance according to LTE and WiMAX advanced standards. On the other hand, cognitive radio $(\mathrm{CR})$ approach provides efficient spectral usage using intelligent wireless nodes. In this thesis, a number of optimal resource allocation strategies for D2D communication networks are investigated using the CR approach.

As a first step, the CR approach in radio access networks is introduced. In the second step, the taxonomy of the RA process in CRNs is provided. For radio resource allocation (RRA), the most crucial task is to associate a user with a particular serving base station, to assign the channel and to allocate the power efficiently. In this thesis, a subcarrier assignment scheme and a power allocation algorithm using geometric water-filling (GWF) is presented for orthogonal frequency division multiplexing (OFDM) based CRNs. This algorithm is proved to maximize the sum rate of secondary users by allocating power more efficiently. Then, the RA problem is studied to jointly employ CR technology and D2D communication
\end{abstract}


in cellular networks in terms of spectral efficiency (SE) and energy efficiency (EE). In the first case, in terms of SE, a two-stage approach is considered to allocate the radio resource efficiently where a new adaptive subcarrier allocation (ASA) scheme is designed first and then a novel power allocation (PA) scheme is developed utilizing proven GWF approach that can compute exact solution with less computation. In the second case, in terms of EE, the power allocation problem of cellular networks that co-exist with D2D communication considering both underlay and overlay CR approaches are investigated. A proven power allocation algorithm based on GWF approach is utilized to solve the EE maximization problem which results in an "exact" and "low complexity" solution. 


\section{Acknowledgements}

This dissertation was written under the guidance and with the help of my two supervisors, Dr. Lian Zhao, and Dr. Xavier Fernando. Their valuable advice and extended knowledge were a great help. I learned from their precious research experiences and how it combines with and applies to wireless communications, especially on resource allocation strategies. I would like to express my sincere gratitude to both of my supervisors, for their continuous guidance and support. I am also grateful to my friends, teachers and classmates.

My special thanks go to all the members of the examination committee: Dr. Beheshti, Dr. Fang, Dr. Sedaghat, and Dr. Woungang (in alphabetical order).

Finally, I am indebted to all the members of my family, especially my husband and daughter, for their patience, understanding and continuous support. 


\section{Table of Contents}

Declaration ............................. ii

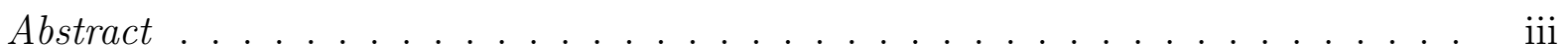

Acknowledgements .......................

List of Tables . . . . . . . . . . . . . . . . . . ix

List of Figures . . . . . . . . . . . . . . . . . . $\mathrm{x}$

List of Appendices . . . . . . . . . . . . . . . . . . . xiii

1 Introduction $\quad 1$

1.1 Cognitive Radio System _ . . . . . . . . . . . . . . . . . . . 2

1.2 Device-to-device (D2D) Communication . . . . . . . . . . . 6

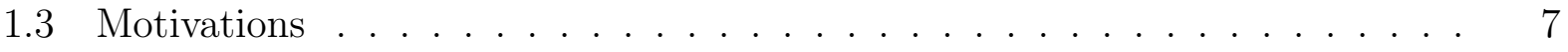

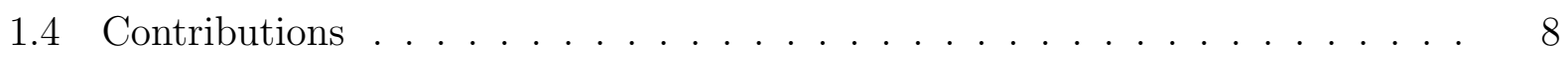

1.5 Thesis Organization . . . . . . . . . . . . . . . . . . . . . . 10

2 Resource Allocation Problems and Mathematical Preliminaries 12

2.1 Resource Allocation Approaches . . . . . . . . . . . . . . . . . . . . . . . . 13

2.2 Architecture . . . . . . . . . . . . . . . . . . . . . 15

2.3 Resource Allocation and Design Objectives . . . . . . . . . . . . . . 17

2.4 Solution Approaches and Techniques _. . . . . . . . . . . . . 20

2.4 .1 Heuristic . . . . . . . . . . . . . . . . . . . . . . . . . 20

2.4 .2 Optimization . . . . . . . . . . . . . . . . . . . 21 
2.4 .3 Graph Theory . . . . . . . . . . . . . . . . . . . . . . . . 23

2.4 .4 Game Theory . . . . . . . . . . . . . . . . . . . . . . . . 25

2.4 .5 Genetic Algorithms . . . . . . . . . . . . . . . . . . . 27

2.4 .6 Particle Swarm Intelligence Algorithms . . . . . . . . . . . . 27

2.4 .7 Geometric Water-Filling Method . . . . . . . . . . . . . . 28

2.5 Chapter Summary . . . . . . . . . . . . . . . . . . . . . . . . 31

3 Resource Allocation in OFDM-based Cognitive Radio Systems 32

3.1 Introduction . . . . . . . . . . . . . . . . . . 33

3.2 Cognitive Radio System Model and Transmission Power Constraint . . . . . 36

3.3 Problem Formulation . . . . . . . . . . . . . . . . . . . . . . . 38

3.4 Subcarrier Allocation . . . . . . . . . . . . . . . . . . . . 40

3.5 Power Allocation using GWFPP . . . . . . . . . . . . . . . . . . 42

3.5.1 Iterative Partitioned Weighted Geometric Water-Filling with Individual Peak Power $(\mathrm{IGPP}) \ldots \ldots \ldots \ldots$. . . . . . . . . . 42

3.6 Performance Evaluation . . . . . . . . . . . . . . . . . . . 43

3.7 Chapter Summary . . . . . . . . . . . . . . . . . . . . . . . . . 48

4 Resource Allocation for Cognitive Radio Systems with D2D Communication $\quad 49$

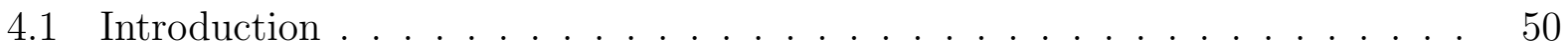

4.2 System Model . . . . . . . . . . . . . . . . . . . . . . . . . . 52

4.3 Problem Formulation . . . . . . . . . . . . . . . . . . . 57

4.4 Proposed Resource Allocation Scheme _. . . . . . . . . . . . . . . 60

4.4.1 Adaptive Subcarrier Allocation (ASA) . . . . . . . . . . . 61

4.4 .2 Power Allocation $(\mathrm{PA}) \ldots \ldots \ldots$

4.5 Performance Evaluation $\ldots \ldots \ldots \ldots$

4.6 Chapter Summary . . . . . . . . . . . . . . . . . . . . . . . 74 
5 Energy Efficient Power Allocation in Underlay and Overlay Cognitive $\begin{array}{lr}\text { Device-to-Device Communications } & 75\end{array}$

5.1 Introduction . . . . . . . . . . . . . . . . . . . . . 76

5.2 System Model . . . . . . . . . . . . . . . . . . . . . . . . . . 77

5.2 .1 Underlay Approach . . . . . . . . . . . . . . . . . . . . . . . 80

5.2 .2 Overlay Approach . . . . . . . . . . . . . . . . . . . . . . . . . 82

5.3 Power Allocation problem and solution . . . . . . . . . . . . . . . . 84

5.3 .1 Problem Formulation . . . . . . . . . . . . . . . . . . 84

5.3 .2 Solution Approaches . . . . . . . . . . . . . . . . . . . . . . . 84

5.4 Performance Evaluation $\ldots \ldots \ldots 9 . \ldots \ldots$

5.4 .1 Simulation Setup . . . . . . . . . . . . . . . . . . . . . 92

5.5 Chapter Summary . . . . . . . . . . . . . . . . . . . . . . 98

6 Conclusion and Future Work $\quad 99$

6.1 Conclusion . . . . . . . . . . . . . . . . . . . . . . . . 100

6.2 Future Research Directions . . . . . . . . . . . . . . . . . . . . . . . . . 102

6.2 .1 Spectrum Sensing Issues _ . . . . . . . . . . . . . . . . . . . 102

6.2 .2 Channel Models . . . . . . . . . . . . . . . . . . . . . . . . . . . . . . 103

6.2.3 Cross-layer Design Approaches and Security Issues _ . . . . . . . . 104

6.2 .4 Spectrum Mobility Issues . . . . . . . . . . . . . . . . . . . . . . . . 104

6.2 .5 Multi-hop Scenario . . . . . . . . . . . . . . . . . . . . . 105

6.2 .6 QoS Management in CR Environment . . . . . . . . . . . . . 106

6.2.7 Cognitive Heterogeneous Networks (HetNets) _ . . . . . . . . . 107

$\begin{array}{lr}\text { References } & 133\end{array}$ 


\section{List of Tables}

3.1 Different approaches for power allocation in OFDM-based CRNs . . . . . . 35

3.2 Simulation Parameters . . . . . . . . . . . . . . . . . . . . . . 44

4.1 Comparative analysis of resource allocation optimization problem in cognitive

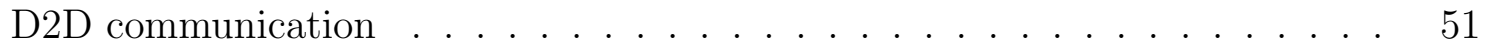

4.2 List of frequently used variables and abbreviations . . . . . . . . . . . 55

4.3 Simulation Parameters . . . . . . . . . . . . . . . . . . 68

4.4 Improvement (in \%) of the proposed scheme on spectral efficiency compared to other schemes. . . . . . . . . . . . . . . . 74

5.1 Simulation Parameters . . . . . . . . . . . . . . . . . . 93 


\section{List of Figures}

1.1 Basic elements of cognitive radio operation. . . . . . . . . . . . . . 4

1.2 Cognitive radio functionalities. . . . . . . . . . . . . . . . 4

2.1 Taxonomy for the resource allocation in CRNs . . . . . . . . . . . . . 13

2.2 Generic resource allocation problem . . . . . . . . . . . . . . . . 18

2.3 Illustration for the proposed GWF algorithm (a) Illustration of $P_{t}(n)$ (shadowed area, representing the total water/power above step $n$ ) when $n=2$, (b) Illustration of water level step $n^{*}=3$, allocated power for the third step $P_{3}^{*}$, and step/stair depth $\Lambda_{i}=\frac{1}{h_{i}}$ and (c) Illustration of the weighted case. . . . . 29

3.1 Cognitive radio system model. . . . . . . . . . . . . . . . . . . 37

3.2 Spectrum of $\mathrm{SU}$ in OFDM-based CRNs . . . . . . . . . . . . . . . 37

3.3 Flow chart for subcarrier aliocation. . . . . . . . . . . . . . . . 41

3.4 Power allocation using Iterative Partitioned Water-Filling Method . . . . . . 44

3.5 Power allocation using Geometric Water-Filling Method with Peak Power

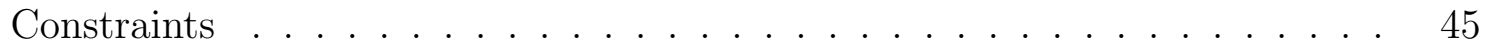

3.6 Power allocation using Iterative Partitioned Weighted Geometric Water-Filling with Individual Peak Power Constraints Algorithm . . . . . . . . . . . . . . 45

3.7 Power allocation by IGPP considering weight factors . . . . . . . . . . . . 46

3.8 Comparison of the sum rates for IPWF, GWFPP and IGPP . . . . . . . 47 
3.9 Optimal power allocation vs subcarriers for each subchannel using IPWF, GWFPP and IGPP . . . . . . . . . . . . . . . . . 48

4.1 A D2D enabled cognitive radio system. . . . . . . . . . . . . 53

4.2 Spectrum distribution of PUs and D2D users in OFDM based cognitive D2D system. . . . . . . . . . . . . . . . . . . . . 53

4.3 The procedure for resource allocation scheme. . . . . . . . . . . . . . . . 61

4.4 Illustration for the GWF algorithm (a) Illustration of water level step $j^{*}=3$, allocated power for the third step $P_{m, n}^{*}(3)$, and step depth is $\frac{1}{\Gamma_{m, n}}$ and (b) Illustration of $P(j)$ (shadowed area, representing the total power above step

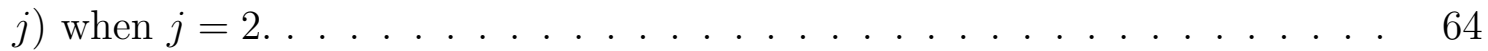

4.5 Flow chart of power allocation algorithm. . . . . . . . . . . . 66

4.6 Illustration of the behavior for the proposed subcarrier assignment with different values of the weighting factors $\left(w_{1}, w_{2}, w_{3}\right) \ldots \ldots \ldots$

4.7 Effect of the distance between the D2D users on subcarrier allocation . . . 70

4.8 Transmission rate of D2D users versus interference threshold to the PUs for different schemes . . . . . . . . . . . . . . . . . 71

4.9 Transmission rate of D2D users versus number of subcarriers with fixed interference threshold for different schemes . . . . . . . . . . . . . . . . 71

4.10 Total power budget versus interference introduced to the PU band for different schemes. . . . . . . . . . . . . . . . . . . 72

4.11 Total transmission rate versus number of D2D users for the different schemes 73 4.12 Performance of different schemes for different minimum rate requirements . . 73

5.1 A D2D enabled cognitive radio system. . . . . . . . . . . . . . 78 
5.2 Illustration for the GWF algorithm (a) Illustration of water level step $j^{*}=3$, allocated power for the third step $P_{m, n}^{*}(3)$, and step depth is $\frac{1}{\Gamma_{m, n}}$ and (b) Illustration of $P(j)$ (shadowed area, representing the total power above step

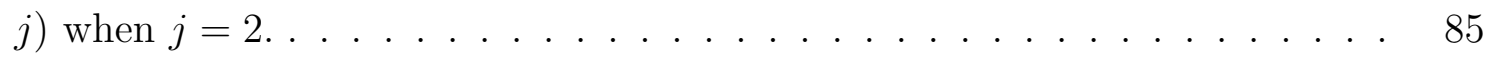

5.3 Illustration of geometric relation and auxiliary variable $\Delta P \ldots \ldots \ldots$. . . 88

5.4 Illustration for solving conditions $(5.31) \ldots \ldots \ldots \ldots$. . . . . . . . 89

5.5 Flow chart of EE-GWF algorithm. . . . . . . . . . . . . . . . 90

5.6 Energy Efficiency versus transmission power of the D2D users with underlay approach . . . . . . . . . . . . . . . . . . . . . . . 93

5.7 Energy Efficiency versus transmission power of the D2D users under overlay approach . . . . . . . . . . . . . . . . . . . . . . 94

5.8 Energy Efficiency versus the distance between the D2D pair $\ldots \ldots$. . . . 95

5.9 EE performance versus the transmission power of the D2D users . . . . . . 95

5.10 EE performance versus the number of the D2D users $\ldots \ldots \ldots$

5.11 EE performance of the D2D users versus the number of the channels . . . . 96

5.12 Total power consumption versus the number of the D2D users $\ldots \ldots$. . . 97 


\section{List of Appendices}

1 Acronyms 108

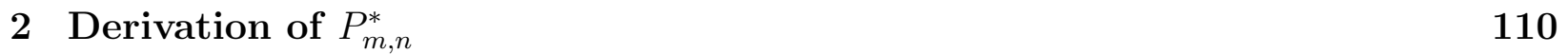


Chapter 1

\section{Introduction}


The use of cognition in radio access and networking is expected to enable number of significant enhancements in wireless communications. In addition to better spectrum utilization, other areas such as autonomous network configuration, interference reduction, energy efficiency, interoperability and coexistence among different wireless communications systems and devices can also benefit from a cognitive approach. The cognitive and adaptive capabilities of radio access will be of fundamental importance in fifth generation $(5 \mathrm{G})$ and beyond networking contexts especially with heterogeneous networking and massive multiple input, multiple output (MIMO) scenarios. Cognitive networking will enable future networks to become more adaptive, self- configuring, self-organizing, self-healing, and self recovering. It will enable networks to cope with environmental changes, network dynamics, and malicious attacks, etc [1].

\subsection{Cognitive Radio System}

Ever increasing demand for myriad of wireless services poses two major challenges in the wireless network paradigm. One is the spectrum scarcity and the other is the demand of high data rates, up to tens of Gbps. While there is continuous effort to allocate more spectrum for wireless usage, it is observed that currently licensed spectrum is significantly underutilized $[2,3]$ due to sporadic transmission nature of most communication devices.

Cognitive radio (CR) concept was first coined by Joseph Mitola [4], who proposed it as a solution for efficiently utilizing the radio resources. Since a CR transceiver shall have the ability to tune to different frequency bands, the CR is typically built using softwaredefined radio technology. Therefore, the transmitter operating parameters, such as the carrier frequency, modulation type and transmission power can be dynamically adjusted by software $[1,5]$. CRs, with its ability to smartly interact with the surrounding environment, are amenable to allow the coexistence licensed users or primary users (PUs) and unlicensed users or secondary users (SUs) sharing the same bandwidth opportunistically without causing 
harmful interference to each other.

In fact, the coexistence of PUs and SUs in a CR environment are generally categorized into three paradigms:

Underlay: The SU determines the interference caused by their transmission to the PU and transmits only if interference below (under) a given threshold. The interference constraint may be met by using wideband techniques such as spread spectrum or ultra wide band (UWB) or by directional radiation.

Interweave: Interweave systems completely avoid interference by not transmitting in a band that is occupied by the PU.

Overlay: In overlay systems, simultaneous transmission is allowed. Here, the cognitive user has the knowledge of PUs message and/or encoding strategy. Simultaneous communication is achieved via various encoding and interference mitigation schemes.

CRNs have distinctive characteristics from a traditional wireless network where it intelligently recognizes the status of the radio environment and adjusts its functional parameters accordingly $[5,6]$. Most critical part of CRN is allowing CR users to share the licensed spectrum with PUs without degrading their performance [7]. This imposes new challenges and open research issues.

The basic elements for the operation of CR systems are depicted in Fig. 1.1 and the main functions of those elements to support intelligent and efficient utilization of frequency spectrum are envisioned in Fig. 1.2.

From Figures. 1.1 and 1.2, it can be seen that the proper functionality of a CRN depends on optimally sensing, accessing and sharing operations of the spectrum. Capacity of CR depends on what white spaces are available and how accurately they are detected. In addition to fading and shadowing, random interference can also cause false detection. Furthermore, $\mathrm{SU}$ transmitter and receiver must coordinate to find common holes for successful transmission. Therefore, the cognitive network throughput is usually difficult to guarantee. Ergodic 


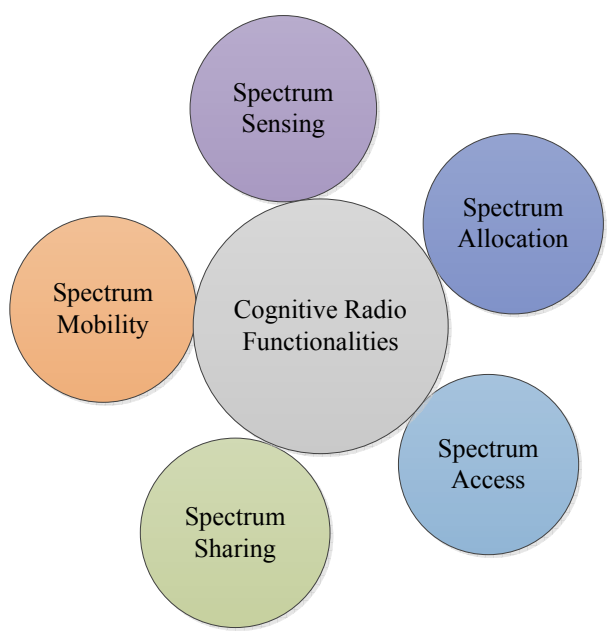

Figure 1.1: Basic elements of cognitive radio operation.

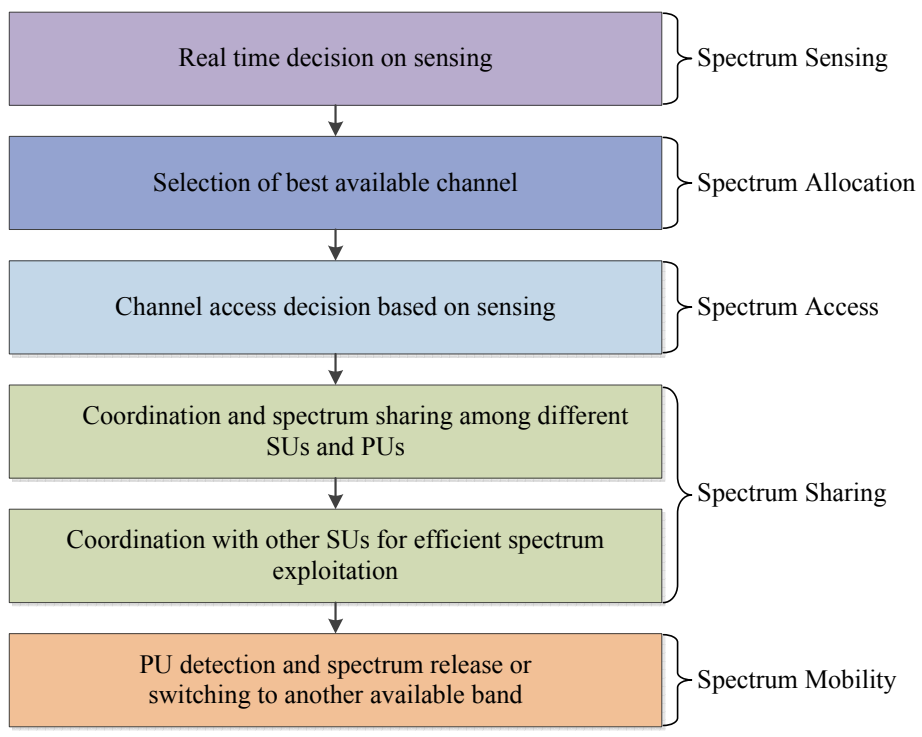

Figure 1.2: Cognitive radio functionalities.

and outage capacity of a CR system depends on the percentage of successfully detected white space which depends on the PU traffic characteristics.

Usually, the SU shall keep sensing the spectrum even when they transmit. However, implementation of this in a satisfactory manner is not easy as most transceivers are not full duplex. This can cause unintended interference to PU. Hence, licensed users don't like interweave SUs even more than underlay SUs. 
Due to the multi-channel nature of $\mathrm{CRN}$, the multi-dimensional nature of the resource allocation (RA) optimization problems become very difficult to solve. In addition to finding the optimal subcarrier set and power allocation for each $\mathrm{SU}$, another challenge would be to avoid the co-channel interference when multiple SUs decide to use the same frequency band. Hence, a better medium access control (MAC) layer shall also cater the co-channel interference among the SUs.

Regulation and standardization efforts have already been carried out to envision some of the applications of CRN. A very important example is IEEE 802.22 Wireless Regional Area Network (WRAN) standard [8]. It provides specifications for broadband wireless access using CR technology and spectrum sharing policies and procedures for operation in the white space TV bands. IEEE 802.11af standard and its amendments [9] enable geo-location database access in the white space radio frequency (RF) spectrum. IEEE 1900.x series of standards [10] provide next generation radio and advanced spectrum management. IEEE 802.19 standard [11] enables the family of IEEE 802 wireless standards to most effectively use TV white space by providing standard coexistence methods among dissimilar or independently operated IEEE 802 networks and devices. It is also useful for non IEEE 802 networks and TV band devises. The first set of standardization study towards licensed shared access for long term evaluation (LTE) is reported in [12] that were successfully tested in a live LTE network in the 2.4-2.5 MHz frequency band [13]. In most recent times, IEEE has engaged the 802.15.4m task group [14] to characterize cognitive radio-aware PHY and MAC layers for cognitive machine-to-machine networks. These examples show standardization process for CR is well underway.

Latest developments in spectrum allocation policy and regulatory domains, including the release of the National Broadband plan, the publication of final rules for TV-white spaces, and the ongoing proceeding for secondary use of the 2,3602,400 $\mathrm{MHz}$ band for medical body area networks, have opened up various opportunities for the secondary use of spectrum. CR is therefore addressed to enable and support a variety of emerging applications, ranging from 
smart grid, public safety and broadband cellular, medical applications to WSNs. The wide range of $\mathrm{CR}$ applications have various design requirements. For instance, from the data rate point of view, a temperature sensor may have a very low data rate whereas cellular handset may have a very high data rate. The IEEE 802.15.4 standard supports different data rates varying from $20 \mathrm{Kbps}(868 \mathrm{MHz}$ band) to $250 \mathrm{Kbps}$ ( $2.4 \mathrm{GHz}$ band).

\subsection{Device-to-device (D2D) Communication}

D2D communication is one of such paradigms that appears to be a promising component in next generation cellular technologies. D2D communication in cellular networks is defined as direct communication between two mobile users without traversing the base station (BS) or core network. In a traditional cellular network, all communications must go through the BS even if both communicating parties are in range for D2D communication. This architecture suits the conventional low data rate mobile services such as voice call and text message in which users are not usually close enough to have direct communication. However, mobile users in todays cellular networks use high data rate services (e.g., video sharing, gaming, proximity aware social networking) in which they could potentially be in range for direct communications (i.e., D2D). Hence, D2D communications in such scenarios can highly increase the spectral efficiency of the network. Nevertheless, the advantages of D2D communications are not only limited to enhanced spectral efficiency. In addition to improving spectral efficiency, D2D communications can potentially improve throughput, energy efficiency, delay, and fairness [15].

D2D communication is generally non-transparent to the cellular network and it can occur on the cellular spectrum (i.e., inband) or unlicensed spectrum (i.e., outband). In inband D2D communication, D2D users can setup communication through underlay or overlay spectrum sharing method. In underlay D2D communication, D2D users use the same spectrum of cellular users whereas in overlay, D2D users use a dedicated portion of cellular spectrum 
[15]. The most crucial part of underlay inband method is to reduce the interference between cellular and D2D users as they share the same radio resources [16]. In the overlay inband method, D2D users occupy the dedicated portion of spectrum of cellular users, which is not an efficient approach in terms of spectrum efficiency. Other researchers propose to adopt outband rather than inband D2D communications in cellular networks so that the precious cellular spectrum be not affected by D2D communications [15]. In outband communications, the coordination between radio interfaces is either controlled by the BS (i.e, controlled) or the users themselves (i.e., autonomous). Outband D2D communication faces a few challenges in coordinating the communication over two different bands because usually D2D communication happens on a second radio interface (e.g., WiFi Direct [17] and Bluetooth [18]).

\subsection{Motivations}

Enhancing spectrum efficiency and energy efficiency are the most significant tasks for the regulatory authorities worldwide. Different measurement studies of spectrum utilization from the literature have indicated that spectrum is sporadically used in many geographical areas and times [2]. Low utilization and increased demand for the radio resources suggest the notion of the second time utilization of the spectrum, which allows unused parts of the spectrum to become temporarily available for the commercial purposes. This promising idea can mitigate unsatisfied spectrum demand, potentially without major changes to incumbents. It also provides us the ability to consider basic obstacles to the SUs, including why PUs would allow SUs and, equally important, under what circumstances SUs might emerge. The reality of identifying the pre-conditions for the SUs is a crucial step towards higher level of efficient spectrum utilization.

$\mathrm{CR}$ approach is well known to solve the under-utilization issue of the radio spectrum. However, CR technology only improves spectrum efficiency from the perspective of time 
and channel, neglecting the potential optimization from the space perspective [19]. On the other hand, D2D technology $[20,21]$ has been proposed as an effective solution for efficiently utilizing the scarce radio resources from the space perspective. Both CR technology, and D2D communication must not cause harmful interference to cellular networks.

Now, the question arises, can D2D communication be better optimized using the cognitive radio approach? Can D2D users be modeled as secondary cognitive users and opportunistically access the under-utilized radio spectrum to increase the spectral efficiency? Can D2D systems be modeled considering both underlay and overly CR approaches to maximize the energy efficiency? These are the focus of this thesis. Note that, there are key differences between CR and original D2D systems. Mainly, the D2D communication is mostly managed by the network (that provides the spectrum) while a CR system is managed by the $\mathrm{CR}$ terminals distributively by spectrum sensing and interference-aware decisions [22], [23].

Driven by the aforementioned motivations, the major objective of this research is to investigate RA schemes for CRNs and how D2D communication can coexist with CR technology in order to optimize both the spectral efficiency and the energy efficiency (EE) with a more practical insight.

\subsection{Contributions}

\section{\# Taxonomy for the Resource Allocation in CRNs:}

- The RA problems are categorized and discussed according to the adopted approaches and network architecture.

- Basic elements or objectives of the RA problem are well studied to provide the RA design structure.

- The optimization strategies for the RA are explored and reviewed in the context of CRNs. Then, a comprehensive overview of these categories and optimization strategies 
is provided, and also highlighted the advantages, disadvantages, and the application areas associated with each optimization strategy.

\section{\# Resource Allocation in OFDM-based Cognitive Radio Systems:}

- Power allocation problem in OFDM-based CRNs is modeled considering both individual subcarrier peak power constraints and subchannel (group of subcarriers) power constraints with total power constraint.

- A more efficient algorithm, named as iterative partitioned weighted geometric waterfilling with individual peak power constraints (IGPP) is proposed along with subcarrier assignment.

- The proposed algorithm is shown to maximize the sum rate by providing better utilization of power resources than the existing algorithms. Power allocation is also performed considering the weight factor of each channel.

\section{\# Resource Allocation for Cognitive Radio Systems with D2D Communica- tion:}

- An OFDM based cognitive cellular network with D2D communication has been modeled and analyzed using Lagrange formulation, and then solved using geometric waterfilling (GWF) method. In order to maximize the transmission rate of the D2D users, the optimization task is characterized by the following five features: a) total power constraint, b) peak power constraint on each subcarrier, c) maximum power constraint of each D2D user, d) interference constraint to the PU band and e) minimum transmission rate requirements for the D2D users.

- A two-stage approach is considered to allocate the radio resource efficiently. In the first stage, a new adaptive subcarrier allocation (ASA) scheme is designed based on three parameters that form a well-designed metric. These parameters are exploited as 
the weighting factors to make trade-off among the amount of power, interference and transmission rate. Then, a novel power allocation scheme is developed utilizing proven GWF approach that outperforms other existing algorithms in the literature.

\section{\# Energy Efficient Power Allocation in Underlay and Overlay Cognitive Device-to-Device Communications:}

- For a cognitive D2D system, two approaches (underlay and overlay) are considered to manage the spectrum sharing among the PUs and SUs in complementary scenarios.

- The power allocation problem for such a scenario is formulated as the maximization of the D2D users EE subject to the minimum rate requirement for both the D2D users and the cellular users (PUs).

- A proven power allocation algorithm based on GWF approach has been utilized to solve the EE optimization problem that outperforms the other existing algorithms.

\subsection{Thesis Organization}

The remainder of the thesis is organized as follows:

Chapter 2: Taxonomy for the Resource Allocation in CRNs. Presents the RA problems those are categorized first according to the adopted approaches and network architecture. Then, the optimization strategies for the RA are explored and reviewed in the context of CRNs. Finally, a comprehensive overview of these categories and optimization strategies is provided, and also highlighted the advantages, disadvantages, and the application areas associated with each optimization strategy.

Chapter 3: Resource Allocation in OFDM-based Cognitive Radio Systems. Provides the system model for OFDM-based CRNs and construct the power allocation problem considering three constraints. Describes the methods for the solution of power allocation 
problem utilizing the GWF scheme along with the subcarrier allocation. Also provides comparison of the proposed algorithm results with the related works.

Chapter 4: Resource Allocation for Cognitive Radio Systems with D2D Communication. Provides the system model to jointly employ CR technology and D2D communication in cellular networks and construct the RA problem considering five constraints. Describes a two-stage approach where a new ASA scheme is designed first and then, a novel PA scheme is developed utilizing GWF approach. Present simulation results that validate the proposed solutions than the existing schemes.

Chapter 5: Energy Efficient Power Allocation in Underlay and Overlay Cognitive Device-to-Device Communications. Investigates the energy-efficient power allocation problem of co-existing D2D and cellular users considering both underlay and overlay $\mathrm{CR}$ approaches. In both the (underlay and overlay) spectrum sharing schemes, the optimization problem is formulated as the EE maximization of the D2D users subject to the minimum rate requirement for both the D2D users and the cellular users. A proven power allocation algorithm based on the GWF approach is utilized to solve this EE optimization problem. Contrast to the widely used Dinkelbach method for EE maximization, this algorithm can directly compute the optimal solution by applying the geometry-based approach, with exactness and low-degree polynomial complexity. Simulation results reveal the benefits of the proposed algorithm.

Chapter 6: Conclusion and FutureWork. Includes a summary of the research work, its outcomes, possible directions and timeplan for future research work. 
Chapter 2

Resource Allocation Problems and Mathematical Preliminaries 
In this chapter, a taxonomy for the resource allocation (RA) process in CRNs is provided. In particular, the RA problem is categorized first according to the adopted approach which could be centralized, or distributed. Second, based on the network architecture, the RA problem is also classified as infrastructure based or ad-hoc. Third, according to the problem objective, the basic elements of RA in CRNs may include: throughput (sum-rate), power, delay, QoS, fairness etc. Finally, the algorithms are also classified according to the strategy used for solving the problem which can be an optimization technique, heuristic, game theory, graph theory etc. Fig. 2.1 provides the adopted taxonomy in detail.

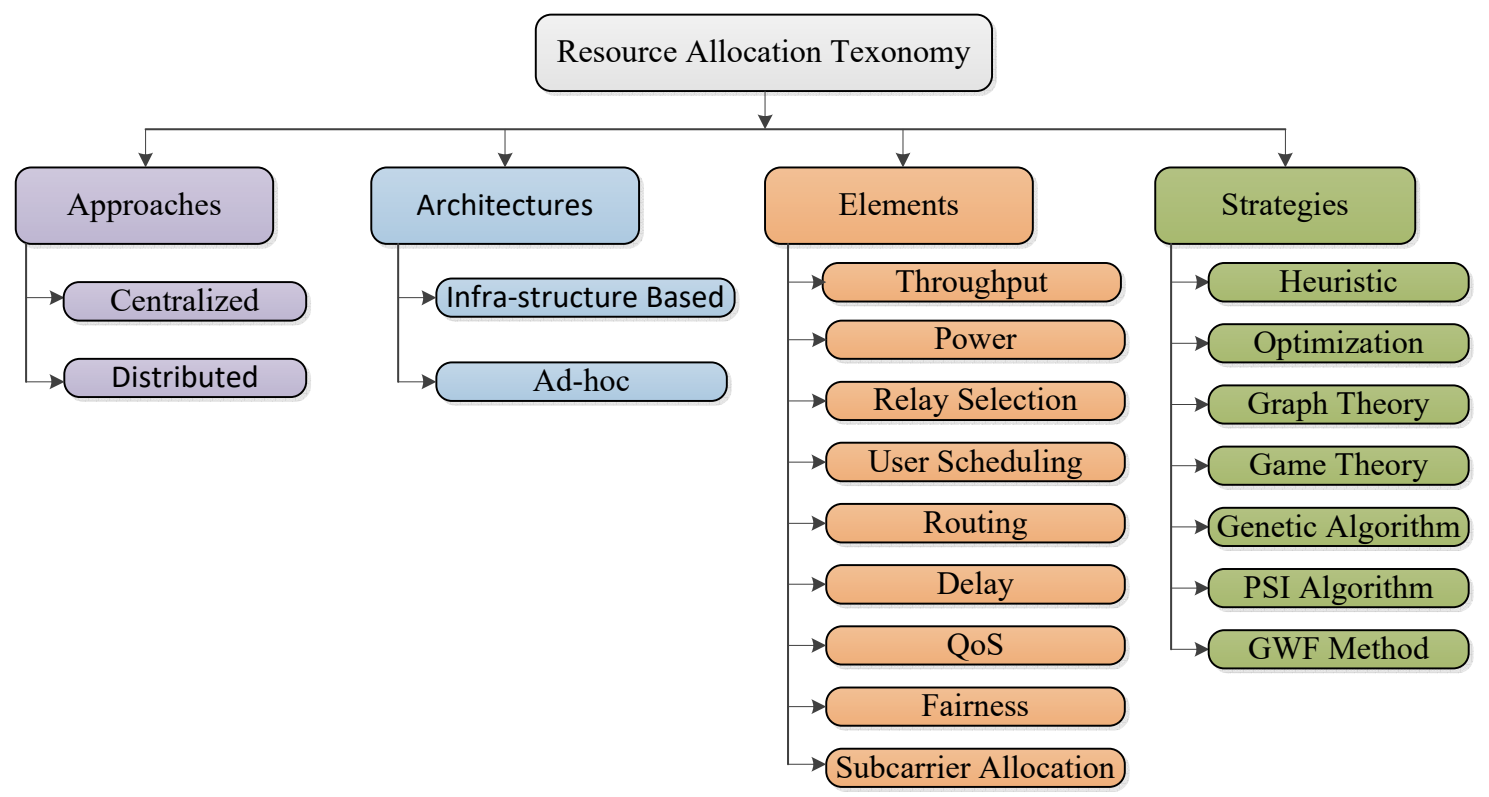

Figure 2.1: Taxonomy for the resource allocation in CRNs

\subsection{Resource Allocation Approaches}

In the literature, two key approaches are utilized to solve the RA problem: centralized and distributed. Each of these approaches has its merits and demerits. So that, depending on the problem requirement and priorities one of them could be adopted.

1) Centralized: The centralized approach mainly based on the existence of a central 
entity that handles the RA process. This entity could be a base station (BS), eNodeB, or separate node for control purposes (server). In the centralized RA or scheduling algorithms, the central node gathers the measurements and information from the whole network, transmits control information to different users and nodes to coordinate their access, and takes the final decision. This approach has been widely investigated in literature (e.g., [24-30]). It was shown that the centralized schemes have several advantages. Due to the global view of the whole network, this approach is able to obtain the optimal solution of a desired performance metric (e.g., maximize the network sum rate, spectrum efficiency, etc.). Also, it can optimally minimize the network interference and attain an overall better network performance. Moreover, it can easily achieve optimal fairness since all the network information is available at one node. This can be done by regulating greedy nodes that devour large portion of the resources on the cost of other weak nodes. Moreover, centralized controllers can control priorities more efficiently. In contrast, there are some disadvantages that limit the practicality of the centralized approach. In particular, centralized schemes create large signaling overhead which results in a wasteful utilization of the resources (e.g., bandwidth, power, etc.). Also, due to the adopted nature which directs to make the RA process of the whole network based strongly on a single entity (central node), the failure of this central node can cause severe effects on the network performance. In a failure case, which may arise from power failure or crashes, each node in the network will independently make the RA decision. Consequently, the network performance could be significantly deteriorated. Furthermore, contention and unfairness situation could be attained.

2) Distributed: In the distributed approach, there is no central entity that manages the scheduling process. Instead, each node (or user) takes its decision autonomously or via a limited cooperation by exchanging neighboring information. In particular, each node carries out some measurements and calculations that are enough to reach the decision or should be shared among its neighbors to take the decision. Several distributed approaches have been taken into account in many researches (e.g., [31-34]). Flexibility is a major advantage 
of this approach over the centralized one. Distributed schemes quickly adapt to wireless environment variations. This is due to the fact that only the nodes in the area of the variation are affected and require to amend their calculations. Therefore, there is no need to replicate the overall RA process, but only the affected part. This definitely involves the fast recovery feature which is crucial, for example, in emergency situations. This contradicts the centralized case, where any change affects the whole process and delays the decisions. Thus, distributed approaches present more robust communications. Another advantage is that distributed approaches decrease the amount of overhead and delay of switching information as a result of being only limited to a small neighboring area. In comparison, distributed approaches cannot acquire the optimal solution for the desired performance metric due to the lack of information at each node. Moreover, only local fairness between neighboring nodes can be attained. Finally, distributed approaches are more appropriate for light load networks but for high load networks a centralized approach can achieve better performance.

\subsection{Architecture}

1) Infrastructure Based: This model incorporates the existence of a base station and multiple users connected to it. The network can be driven either in downlink or uplink modes, and offers one-hop communications. Generally, centralized techniques are utilized for this model to employ the central entity. The users observations and measurements are fed back to the central point to take the decisions. Then, the users configure their parameters according to the central decision. This model is useful in case of setting up a permanent network. In CRNs, using proper RA strategies the base station can significantly help in protecting the primary network from secondary transmissions. Cognitive radio cellular networks (CRCN) [35,36], and IEEE 802.22 wireless regional area network (WRAN) [37,38] are examples of such networks. Although most of the strategies in literature for this model are centralized, distributed schemes can also be supported [39]. 
2) $\boldsymbol{A d}$-Hoc: In the ad-hoc model, also called infrastructureless model, the communication is performed directly between the CR nodes without the support of a central controller or a base station. Unlike infrastructure based model where the nodes communicate with the base station via single hop, the communication between the nodes of an ad-hoc network could be single-hop or multi-hop communications. In a multi-hop network, routing is an essential function to find the best route for data transmission. Indeed, the use of distributed techniques is more appropriate for this network model as the ad-hoc networks are distributed by nature $[40,41]$. This includes extra challenges on the communicating nodes of the adhoc networks compared to those of infrastructure based since the nodes have to coordinate their access themselves. As a result, the operational load increases on the CR nodes whose resources, such as power, are limited which enforces a critical demand for computationally efficient methods.

Ad-hoc networks have several advantages. For instance, ad-hoc networks are easier to set up because the nodes are connected directly without an access point. Besides the ease of deployment, ad-hoc networks decreased dependence on infrastructure allows for fast integration with already existing infrastructure networks. This makes wireless ad-hoc networks applicable and useful in many situations such as crisis response, military environments and sensor networks [40]. In CRNs, ad-hoc networks were broadly adopted (e.g., [41,42]). Dynamic network topology, distributed multi-hop architecture, and time and location varying spectrum availability are some prime factors of CR ad-hoc networks (CRAHNs) [41]. The specific functions associated with CRAHNs were discussed in [43]. The authors described that these functions are highly dependent on cooperation among the users due to the lack of a central coordinator. Therefore, RA is done based on local observations of each user and by exchanging information with the neighboring nodes. 


\subsection{Resource Allocation and Design Objectives}

Fig. 2.2 shows the generic RA problem in CRNs. A generic RA design structure consists of four portions: (1) inputs; (2) outputs; (3) objectives; and (4) constraints. Different input parameters are provided by network administrator or regulatory authorities. In CRNs, central controller generally knows about the SUs and relays in the network. In case of primary and secondary network cooperation, it is possible that CRNs central controller has the information about the number of PUs and their respective geographical locations. Interference threshold is set by the regulatory authorities. Value of interference threshold depends on the spectrum sharing regime. Knowledge of channel state information (CSI) is a significant input parameter. Most of the RA algorithms assume that CSI is known at both transmitter and receiver. Under different constraints, the RA schemes are analyzed and evaluated where performance is measured in different metrics in order to produce desired outputs.

There are several elements or objectives that could be targeted by the RA problem. These elements may be incorporated as the optimization principle objective or as a constraint that should hold.

1) Throughput: Maximizing the network throughput is a very familiar criterion that is generally targeted in the RA problem. The problem could consider the individual user throughput maximization or the sum-rate of the aggregate network. Also, some efforts consider maximizing the sum-rate of both primary and secondary networks as a whole.

2) Power allocation: Power allocation for CRNs is a crucial task for better interference management. In case of CRNs, the efficient power allocation is more challenging than noncognitive wireless network. Traditional power allocation schemes for non-cognitive networks are not applicable to CRNs as these schemes may cause unacceptable interference to the primary network. In CRNs, power allocation is performed under the constraint of acceptable interference to the PUs.

3) Relay assignment/selection: The use of relays in a CRN can benefit in two ways. 


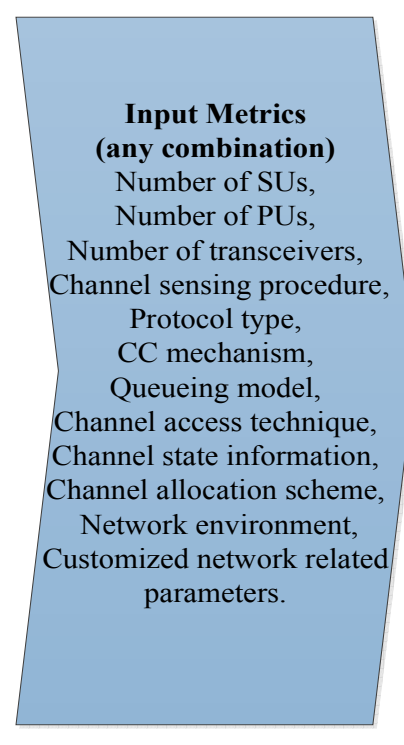

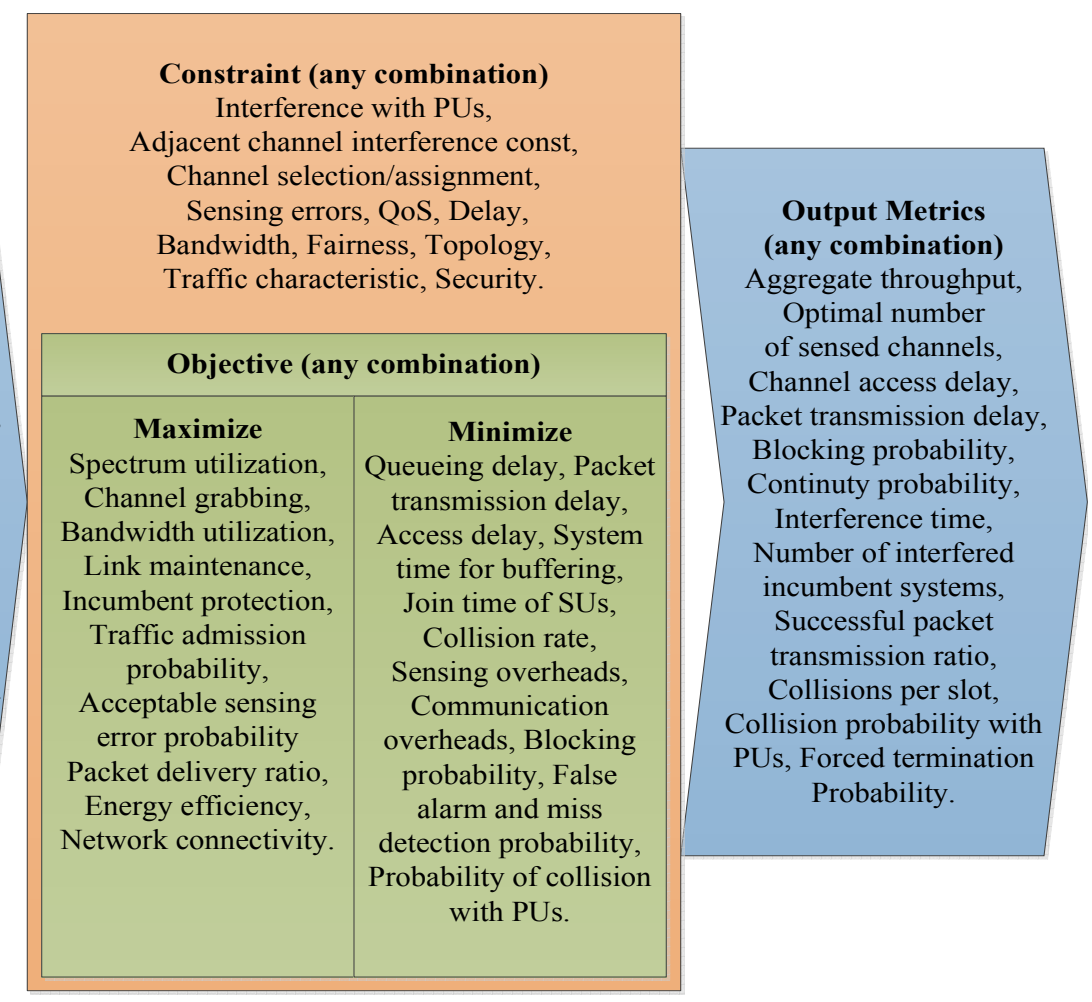

Figure 2.2: Generic resource allocation problem 
First it can increase the transmission rate and, secondly the use of relays can reduce the overall transmission power of the systems. The use of multiple relays simultaneously can further increase the performance of a cognitive radio network. A well designed multiple relay assignment and power allocation scheme can be helpful in two ways. It reduces the interference induced to the primary users in multiuser CRNs and increases the connectivity of the wireless network. In a multiple relay system, if any relay is dead or in deep fade the receiver can still get data from other relays.

4) User scheduling: In multiuser CRNs, due to resource limitations and interference constraints, user scheduling in intelligent way can achieve high throughput. User scheduling schemes select the best group of users at each time slot to maximize the total throughput. The complexity of an exhaustive search for user scheduling increases exponentially with the number of users. For example, if $\mathrm{U}$ is the total number of users, then the number of possible ways of scheduling/selecting $u$ users is $\left(\begin{array}{l}U \\ u\end{array}\right)$. Enumerating all possible combinations to find the one that gives the best performance is computationally inefficient. Due to the high computational complexity of the optimal selection (e.g., exhaustive search algorithm), efficient user scheduling in CRNs is an active area research.

5) Routing: Most of the research on CRNs to date has focused on one or two-hop scenarios. With the advancement on ad hoc networks, recently, researchers have started to realize the importance and potential of multi-hop CRNs. To get the benefits of multi-hop transmission, new challenges must be addressed and solved. In particular, efficient routing techniques and solutions must be integrated into the ad hoc CRNs.

6) $\boldsymbol{Q} \boldsymbol{o} \boldsymbol{S}$ : QoS is a general term used for many user satisfaction related requirements. It comprises response time, throughput loss, rate requirements, outage and blocking probabilities. The main aim of QoS in CRNs is to guarantee a minimum rate, reduction in latency jitter and packet errors.

7) Delay: Delay is an important metric in any wireless network especially for realtime applications such as voice and multimedia. Delay performance in CRNs has important 
engineering implications, e.g., it can be used to characterize the number of SUs that can be supported under a given delay constraint. Queuing delay and transmission delay need to be analyzed in order to characterize the delay performance, which is still an unexplored area of research.

8) Subcarrier allocation: Subcarrier allocation and pairing play a significant role in future CRNs that employs orthogonal frequency division multiplexing (OFDM) in physical layer. One can increase the throughput of CRNs with the intelligent utilization of subcarriers.

\subsection{Solution Approaches and Techniques}

In this section, we explore and review the optimization strategies for RA in the context of CRNs. Depending upon the objectives, these strategies can be categorised under several performance optimization criteria. In the following, we provide a comprehensive overview of these categories and optimization strategies, and highlight the advantages, disadvantages, and the application areas associated to each of them.

\subsubsection{Heuristic}

In some cases it is tremendously tough, or even not possible, to get the optimal solution of certain problems. Hence, one may impose non-realistic assumptions to simplify the problem structure, and then find the optimal solution for the simplified structure. However, it is rather better to look for a reasonable (non-optimal) solution for the real problem structure than searching the optimal solution for inaccurate oversimplified structure. Heuristic strategies give logical solutions, that are not guaranteed to be the optimal, with acceptable time and space complexity [44]. Additionally, heuristic strategies are less restrictive than optimization methods, and thus they allow the usage of structures that are more presentable to the real world problems [45]. For CRNs, optimal solutions for RA problems are typically of high complexity. Hence, heuristic strategies are appropriate to find a fine solution 
quickly. Usually, there is no methodical structure for developing heuristic strategies. Thus, researchers build up their own methods according to the problems found in the literature [44].

Several efforts (e.g. [46-49]) proposed heuristic strategies for the RA problem in CRNs. The authors in [46] proposed two heuristic distributed RA algorithms based on opportunistic splitting. A heuristic packet transmission scheduling technique was developed in [47] that depends on measured link and interference conditions at CR nodes. In [48], a low complexity huristic algorithm is proposed that efficiently solves the problem of spectrum sharing and downlink user scheduling. A two-step heuristic algorithm for sub-channel allocation and power distribution was developed in [49]. This algorithm extensively decreases the problem complexity while presenting an excellent approximate solution near optimal.

It is meaningful to mention that one disadvantage of heuristic strategies is that they are problem-specific and could not be universal. Also, there is no guarantee for convergence, especially for iterative strategies that may trapped into local optimal points far from the global optimal solution [18]. However, they stay on an essential substitution that can offer reasonable, fast, and yet easy solutions to be employed in many conditions. Additionally, heuristic solutions are more robust to problem variations than optimal solutions [57].

\subsubsection{Optimization}

Optimization problems intend to search the optimal of all possible solutions, which is the one that minimizes or maximizes a certain objective function. This objective function is a function used to assess a quality of the generated solution. The general mathematical structure of an optimization problem can be written as [50]:

minimize $f_{0}(x)$

subject to: $f_{i}(x) \leq b_{i}, \quad i=1, \ldots, m$ 
where the problem components are as follows:

- $x=\left(x_{1}, \ldots, x_{n}\right)$ : optimization variables

- $f_{0}: \mathbb{R}^{n} \rightarrow \mathbb{R}:$ objective function

- $f_{i}: \mathbb{R}^{n} \rightarrow \mathbb{R}, i=1, \ldots, m$ : constraint functions

- and the optimal solution $x^{*}$ has the smallest value of $f_{0}$ among all vectors that satisfy the constraints

There are numerous types of objective functions occurring in RA for CRN of which three emerge to be very famous. Most of the other types are related to those three. The three are:

- Maximization of network sum throughput,

- Maximization of overall user data rate,

- Maximization of user capacity.

The other types of objective functions that have been studied in the literature include; maximization of spectrum utilizations, maximization of total channel utility, maximization of fairness scaling factor, maximization of downlink channel capacity, minimization of total transmission power, and minimization of total power consumption. Even the three stated earlier appear to have been utilized interchangeably by authors. Thus, all those three objective functions are combined together into a class of "capacity"measure. As for constraints, there are three that are usually occurring and they are:

- Power constraint,

- Constraint on interference,

- Minimum data rate for users.

The most commonly occurring decision variables are: 
- Transmission power,

- Sub-channel allocation, which results in a zero-one decision variable.

In CRNs, optimization problems are generally used to formulate the RA problem for efficient utilization of the available resources. The formulated problems could be convex, non-convex, linear, or non-linear with different objectives such as sum-rate maximization, power minimization, fairness, etc. Certainly, complexity depends on the optimization problem model. For instance, convex optimization problems can be solved using standard optimization methods. Furthermore, a linear program (LP), wherein the objective function and constraints are all linear, is generally solved through iterative optimization problem solvers with less complexity and higher speed as compared to non-linear convex optimization problems with similar dimensions [50]. In non-linear programming (NLP), the objective function or some of the constraints are non-linear. For computationally intensive problems, greedy, heuristic algorithms appear as essential alternatives to accelerate the process of obtaining an acceptable approximate solution. For example, incorporating integer constraints in the problem such as the number of allocated time slots, makes it very complicated [51]. Generally, integer and mixed integer non-linear programming (MINLP) are computationally extensive (NP-hard) [52]. Hence, heuristic alternatives are usually utilized.

\subsubsection{Graph Theory}

Graph theory is one of the most extensively used tools for modeling and analyzing the interaction (or contention) in networks. A graph $G$ consists of a set of vertices $V$ and edges $E$, and is denoted by $G=(V, E)$. These components are mapped to the network elements according to the studied problem. Usually, vertices correspond to entities and edges represent the interaction between these entities. The graph theory could be employed to vast range of networks such as mechanical, transportation, and communication networks, among others. It can offer appropriate tools for solving network-related problems. Generally, graph theory 
is utilized when the network structure is known a priori [53]

In wireless communications, graph theory is largely used to solve the scheduling and RA problems, especially for problems of high computational effort [54]. In literature, the RA problem was solved using different types of graphs such as vertex-coloring graph, conflict graph, and bipartite graph $[55,56]$. For CRNs, the SUs are generally mapped to the vertices and the edges mapping varies according the model definition to characterize certain relation between two vertices. For example, in a conflict or vertex-coloring graph model, an edge between two vertices (SUs links) shows that the SUs are in the interference range of each other $[48,54]$. Also, the RA for coloring graph problem is comparable to assigning each vertex a color (i.e., assign each link or SU).

If the vertex set $V$ of a graph $G$ can be split into two disjoint subsets $V 1, V 2$ such that each edge connects two vertices in different subsets, then $G$ is a bipartite graph [57]. A bipartite matching problem is largely used for spectrum assignment and RA in CRNs [5861]. Generally the two partitions of the bipartite graph are mapped to the SUs and the frequency bands available for assignment. The edges connecting two vertices (SU link and frequency band) for such a problem means that the SU requests (or accepts to be assigned) the corresponding frequency band.

In order to be application specific, coloring and conflict graphs are more appropriate for interference-limited environment. This is similar to the cases of having multiple SUs transmit simultaneously, and thus they can generate high interference to each other. Therefore, using the prescribed conflict or coloring graphs, also called node contention graphs (NCG), assists in modeling this interference in a way that makes simpler the development of a proper RA [54,62]. On the other hand, the works adopted bipartite graphs usually allowed one SU per band with the owner PU to limit the interference in the system [58,59]. However, adding preferences to the SUs and PUs guarantees better protection for the primary network as well as improved secondary performance. This is because the PUs preferences are typically set according to their tolerated interference. Certainly, graph-based algorithms cannot integrate 
multiple performance metrics and so, normally, QoS is not guaranteed.

\subsubsection{Game Theory}

Game theory is a mathematical framework utilized to model and analyze the interaction among multiple individuals whose actions affect each other, or possibly who have conflict of interest $[63,64]$. Usually, the game is modeled by considering a set of players (decision makers) who obtain the decisions (or actions) to maximize their payoff (utility). Although it is originally developed for economics, game theory found success in multiple fields such as engineering, biology, political science, physiology, etc [65]. This is due to its advanced theoretical foundations which make it a powerful tool in decision making. Mathematically, the game is denoted as $G\left(\mathcal{N}, \mathcal{A},\left\{u_{i}\right\}\right)$, and the three game components are [65,66]:

- $\mathcal{N}=\{1,2, \ldots, N\}$ represents the finite set of players,

- $\mathcal{A}=A_{1} \times A_{2} \times \ldots A_{N}$ represents the sets of actions such that $A_{i}$ is the set of actions available for player $i$,

- $u_{i}: A \rightarrow \mathbb{R}$ represents payoff/utility function of player $i$, which is a function of actions of all players.

Generally, game theory can be classified into two broad schemes: non-cooperative and cooperative schemes. These schemes have been broadly studied in literature (e.g. [86], [87], [97]). In a non-cooperative scheme, the players act in a selfish manner to maximize their own utility regardless of the impact of this on others. Nash Equilibrium (NE) is commonly known to be the solution point of non-cooperative game [89]. In a cooperative game model, collaboration among the players and information exchanging are the ways to enhance the overall utility of the network and maximize their mutual benefits. Nash Bargaining (NB) is the common solution point for this game model. Also, other game categories exist such as static/dynamic, and complete/incomplete information [86]. 
In wireless communications, game theory is utilized to model and analyze several functions related to different layers (physical, data link, network, etc.) [90]. Usually, game theoretic approaches are used for power control [91], interference management [92], resource allocation [93], network selection in an environment of multiple deployed technologies [94], hand-off management [95], among others. These functions can be employed in the context of wireless sensor networks, CRNs, ad-hoc networks, etc. One major advantage of using non-cooperative game theory in wireless communication is its ability to offer robust and efficient distributed algorithms that rely only on local information and reduce the signaling overhead and computation complexity of the centralized approaches [93], [96]. However, the game model should be formulated carefully to be able to search a stable solution.

Unlike users of conventional wireless networks, CR users are intelligent and have the ability to observe, learn, interact with the surrounding environment, and adjust their parameters accordingly for an optimized performance. However, these users can be belonging to different authorities or have different goals. Thus, cooperation between them cannot be taken for granted. Instead, these users may act selfishly for each one to maximize its own payoff. Generally, an underlay CR spectrum sharing game model includes the following:

- Players: represented by the set of SUs or both SUs and PUs,

- Actions or strategies: depend on the player whether it is an SU or a PU,

- Utilities: an objective function to be maximized such as throughput, SINR, QoS, fairness, revenue, etc.

Thus, game theory occurs as a very important tool that can efficiently model and analyze the spectrum sharing process among the users that have conflict of interests in CRNs. Furthermore, game theory helps to design self-organized algorithms that are more appropriate to the dynamic wireless nature. 


\subsubsection{Genetic Algorithms}

Genetic Algorithm is adaptive search algorithm based on the evolutionary ideas of natural selection [67]. An iterative process starts with a randomly generated set of solutions called population. Best individuals are selected through the utility function (called here fitness function). Then, starting from this subset, a second population is produced through genetic operators: crossover and/or mutation. The new population shares many of the characteristics of its parents, and it hopefully represents a better solution. The algorithm typically terminates when it converges to the optimal solution or after a fixed number of iterations. Genetic algorithms are chosen to solve RA problems due to their fast convergence and the possibility of obtaining multiple solutions. There are quite a few efforts [68-70] are found in the literature that utilizes genetic algorithms to solve RA problems.

\subsubsection{Particle Swarm Intelligence Algorithms}

Particle swarm intelligence (PSI) algorithms are population based stochastic optimization technique inspired by the collective behaviour of social biological individuals (e. g. bird flocking or fish schooling). PSI algorithms model network users as a population of simple agents interacting with the surrounding environment. Each individual has relatively little intelligence, however, the collaborative behaviour of the population directs to a global intelligence, which allows to solve complex tasks. For example, in social insect colonies, different activities are often executed by those individuals that are better equipped for the task. This phenomenon is named division of labour [71]. PSI algorithms are scalable, fault tolerant and moreover, they adapt to changes in real time. There are quite a few efforts [72-75] are found in the literature that utilizes PSI algorithms to solve RA problems. 


\subsubsection{Geometric Water-Filling Method}

The conventional water-filling (CWF) $[76,77]$ problem can be described as follows. Given $P_{T}>0$, as the total signal power (or volume of the water); the allocated power and the propagation path gain for the $i^{\text {th }}$ channel are given as $P_{i}$ and $h_{i}$ respectively where, $i=1 \ldots N$; and $N$ is the total number of subcarriers. Now without loss of generality, if $\left\{h_{i}\right\}_{i=1}^{N}$ be positive and monotonically decreasing, then the optimization problem can be written as:

$$
\begin{aligned}
\max _{\left\{P_{i}\right\}} \sum_{i=1}^{N} \log \left(1+h_{i} P_{i}\right) \\
\text { subject to: } C 1: 0 \leq P_{i}, \forall i ; \\
C 2: \sum_{i=1}^{N} P_{i}=P_{T}
\end{aligned}
$$

where, constraint $\mathrm{C} 1$ is the allocated power to be nonnegative and constraint $\mathrm{C} 2$ is the total power constraint. To find the solution to problem (2.2), we usually start from the KarushKuhn-Tucker (KKT) conditions of the problem, as a group of the optimality conditions. The water level $(\mu)$ needs to be chosen to satisfy the power sum constraints with equality $\left(\sum_{i=1}^{N} P_{i}=P_{T}\right)$ to find the optimal solution.

In [78], GWF approach is proposed to solve the CWF problem and its weighted form. It has two advantages, they are: 1) the geometric approach can compute the exact solution to the CWF, including the weighted case, with less computation and easier analysis without determining the water level through solving the non-linear system, 2) machinery of the proposed geometric approach can overcome the limitations of the CWF algorithm to include more stringent constraints.

Fig. 2.3 gives an illustration of the GWF algorithm proposed in [78]. Suppose there are 4 steps/stairs $(N=4)$ with unit width inside a water tank. For the conventional approach, the dashed horizontal line, which is the water level $\mu$, needs to be determined first and then 


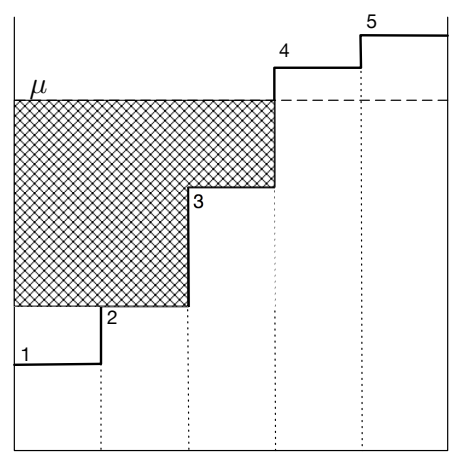

(a)

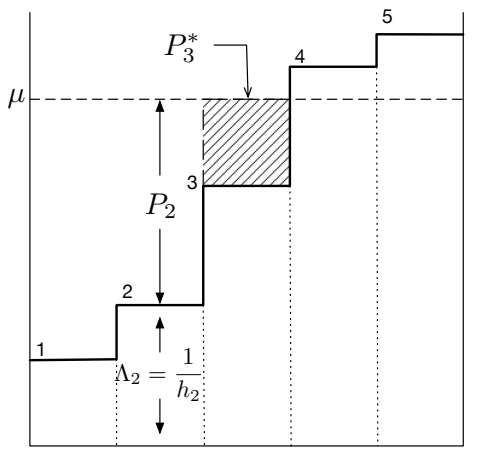

(b)

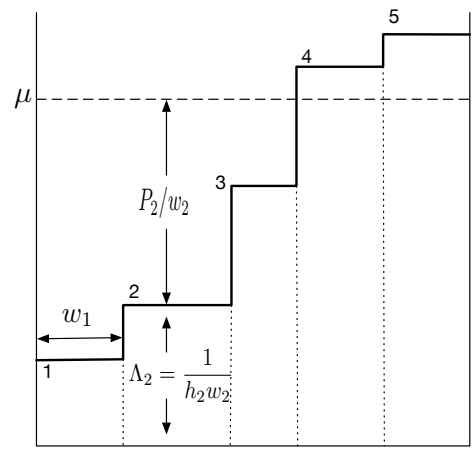

(c)

Figure 2.3: Illustration for the proposed GWF algorithm (a) Illustration of $P_{t}(n)$ (shadowed area, representing the total water/power above step $n$ ) when $n=2$, (b) Illustration of water level step $n^{*}=3$, allocated power for the third step $P_{3}^{*}$, and step/stair depth $\Lambda_{i}=\frac{1}{h_{i}}$ and (c) Illustration of the weighted case.

the power allocated (water volume) above is solved. Let $\lambda_{i}$ denotes the "step depth" of the $i$ th stair which is the height of the $i$ th step to the bottom of the tank, and is given as

$$
\lambda_{i}=\frac{1}{h_{i}} \text { for } i=1,2, \ldots, N .
$$

Since the sequence $h_{i}$ is sorted as monotonically decreasing, the step depth of the stairs indexed as $[1, \ldots, N]$ is monotonically increasing. Now $\delta_{i, j}$ be the "step depth difference" of the $i$ th and the $j$ th stairs, can be expressed as,

$$
\delta_{i, j}=\lambda_{i}-\lambda_{j}=\frac{1}{h_{i}}-\frac{1}{h_{j}} \text { as } i \geq j \text { and } 1 \leq i, j \leq N .
$$

Instead of trying to determine the water level $\mu$, which is a real nonnegative number, the water level step, denoted by $n^{*}$, is the target to solve, that is the highest step under water. Based on the result of $n^{*}$, the solutions for power allocation, can be written instantly. Let $P_{t}(n)$ denote the water volume above step $\mathrm{n}$ or zero, whichever is greater. The value of $P_{t}(n)$ can be solved by subtracting the volume of the water under step n from the total power $P_{T}$, 
as,

$$
\begin{aligned}
P_{t}(n) & =\left\{P_{T}-\left[\sum_{i=1}^{n-1}\left(\frac{1}{h_{n}}-\frac{1}{h_{i}}\right)\right]\right\}^{+} \\
& =\left\{P_{T}-\left[\sum_{i=1}^{n-1} \delta_{n, i}\right]\right\}^{+}, \text {for } n=1, \ldots, N
\end{aligned}
$$

Due to the definition of $P_{t}(n)$ being the power (water volume) above step $\mathrm{n}$, it cannot be a negative number. Therefore we use $\{.\}^{+}$in 4.16 to assign 0 to $P_{t}(n)$ if the result inside the bracket is negative. The corresponding geometric meaning is that the $n^{\text {th }}$ level is above water.

According to [78], the explicit solution to (2.2) is:

$$
P_{i}= \begin{cases}P_{n^{*}}+\left(\lambda_{n^{*}}-\lambda_{i}\right) & 1 \leq i \leq n^{*} \\ 0, & n^{*}<i \leq N\end{cases}
$$

where the water level step $n^{*}$ is given as

$$
n^{*}=\max \left\{n \mid P_{t}(n)>0,1 \leq n \leq N\right\}
$$

and the power level for this step is

$$
P_{n^{*}}=\frac{1}{n^{*}} P_{t}\left(n^{*}\right)
$$

GWF method for the weighted case called weighted geometric water-filling method (WGWF) and weighted geometric water-filling with individual peak power constraints (GWFPP), is also proposed in [78]. 


\subsection{Chapter Summary}

This chapter presents the RA problems those are categorized first according to the adopted approaches and network architecture. Then, the optimization strategies for the RA are explored and reviewed in the context of CRNs. Finally, a comprehensive overview of these categories and optimization strategies is provided, and also highlighted the advantages, disadvantages, and the application areas associated with each optimization strategy. 


\section{Chapter 3}

Resource Allocation in OFDM-based

Cognitive Radio Systems 


\subsection{Introduction}

RA problem in OFDM based CRNs has been widely studied under different settings in the open literature. A power allocation grouping scheme based on the interference channel gain, pulse shape and frequency distance is presented in [79] in order to improve capacity while the interference power for PUs stays at constant level. At the first stage, power is assigned to some groups based on the grouping scheme and at the second stage, the remaining power is allocated to others with water-filling algorithm.

In order to maximize the SUs' transmission capacity by adjusting the power allocation across the subcarriers, two suboptimal schemes for subcarrier allocation with associated triangular and Gaussian power loading mechanism are studied in [80]. Here, these schemes maintain the total power used within the power budget and also the interference at the PUs within a permissible threshold limit.

A low-complexity algorithm using power-increment or power-decrement WF processes is proposed in [81]. This algorithm is based on constraining the total and transmit powers of each subchannel.

In order to maximize the download capacity of CRNs, a subcarrier and power allocation algorithm based on the linear water-filling(LWF) scheme is proposed in [82] while keeping both the total transmit power and interference introduced to the PU below the pre-constraints.

A suboptimal power allocation algorithm for multiuser OFDM-based CRNs is presented in [83] that simplifies the procedure of determining the water level to allocate the power for each subcarrier under a peak power constraint. This method not only reduces the computational complexity but also can easily be combined with a subcarrier allocation algorithm for joint subcarrier and power allocation.

Another suboptimal power allocation algorithm for multiuser OFDM-based CRNs is presented in [84] that simplifies the procedure of determining the water level to allocate the power for each subcarrier under a peak power constraint. This method not only reduces 
the computational complexity but also can easily be combined with a subcarrier allocation algorithm for joint subcarrier and power allocation.

A low-complexity suboptimal power allocation algorithm is proposed in [85] where CR pairs (CRPs) may use both nonactive and active PU pair (PUP) bands as long as the total co-channel interference (CCI) and cross-channel interference (XCI) do not exceed prescribed limits. The complexity reduction is done by making a validated approximation based on the following: 1) the fact that XCI from CRUs to PUPs is mainly limited to a few subchannels adjacent to the PUP bands and 2) the assumption that the bandwidth for a PUP is typically much larger than that of an OFDM subchannel and that there is usually a guard band between two adjacent PUP bands.

An optimal scheme employing joint power allocation in overlay and underlay fashion has been proposed in [86]. The optimal scheme based on the Lagrange formulation maximizes the downlink capacity of CR users while maintaining a total power budget and keeping the interference introduced to the PU band below a threshold. A low complexity suboptimal scheme is also presented where equal low power is allocated to all the underlay subcarriers and a ladder profile is used to load power in the overlay subcarriers.

For weighted sum rate maximization in CRNs, a power limited multilevel WF iterative algorithm has been proposed in [87] where subcarrier power constraints were added to the traditional problem.

With the total power constraint and the power constraint on each subchannel, an iterative partitioned water-filling (IPWF) algorithm was proposed in [88] to realize optimal power allocation in OFDM based CRNs.

Table 3.1 provides different approaches for power allocation in OFDM-based CRNs. 
Table 3.1: Different approaches for power allocation in OFDM-based CRNs

\begin{tabular}{|c|c|c|c|}
\hline Ref. & Objective & Constraints & Solution approach \\
\hline $\begin{array}{l}{[79],} \\
2014\end{array}$ & $\begin{array}{l}\text { Improve capacity while } \\
\text { the interference power } \\
\text { for PUs stays at con- } \\
\text { stant level }\end{array}$ & $\begin{array}{l}\text { Total transmit power } \\
\text { and interference con- } \\
\text { straints }\end{array}$ & $\begin{array}{l}\text { A grouping scheme based on the inter- } \\
\text { ference channel gain, pulse shape and } \\
\text { frequency distance is considered }\end{array}$ \\
\hline $\begin{array}{r}{[80],} \\
2013\end{array}$ & $\begin{array}{l}\text { Maximize transmission } \\
\text { capacity }\end{array}$ & $\begin{array}{l}\text { Total transmit power } \\
\text { and interference con- } \\
\text { straints }\end{array}$ & $\begin{array}{l}\text { Two suboptimal schemes for subcar- } \\
\text { rier allocation with associated triangu- } \\
\text { lar and Gaussian power loading mech- } \\
\text { anism are studied. }\end{array}$ \\
\hline $\begin{array}{l}{[81],} \\
2012\end{array}$ & Maximize capacity & $\begin{array}{lr}\text { Total transmit } & \text { power } \\
\text { and individual } & \text { sub- } \\
\text { channels' } & \text { power } \\
\text { constraints } & \end{array}$ & $\begin{array}{l}\text { A low-complexity algorithm using } \\
\text { power-increment or power-decrement } \\
\text { water-filling processes is considered. }\end{array}$ \\
\hline $\begin{array}{r}{[82],} \\
2011\end{array}$ & $\begin{array}{l}\text { Maximize downlink ca- } \\
\text { pacity }\end{array}$ & $\begin{array}{l}\text { Total transmit power } \\
\text { and interference con- } \\
\text { straints }\end{array}$ & $\begin{array}{l}\text { An iterative suboptimal power alloca- } \\
\text { tion algorithm based on LWF is pro- } \\
\text { posed along with subcarrier allocation. }\end{array}$ \\
\hline $\begin{array}{l}{[83],} \\
2011\end{array}$ & $\begin{array}{l}\text { Maximize overall } \\
\text { throughput while keep } \\
\text { the average inter- } \\
\text { ference to the PU } \\
\text { within a target outage } \\
\text { probability level. }\end{array}$ & $\begin{array}{l}\text { Total transmit power } \\
\text { and interference con- } \\
\text { straints }\end{array}$ & $\begin{array}{l}\text { WF process runs only once, and then } \\
\text { directly calculates the final result with- } \\
\text { out the need for searching the La- } \\
\text { grange multiplier. }\end{array}$ \\
\hline $\begin{array}{l}{[84],} \\
2010\end{array}$ & $\begin{array}{l}\text { Maximize uplink ca- } \\
\text { pacity }\end{array}$ & $\begin{array}{l}\text { Total transmit power } \\
\text { and peak power con- } \\
\text { straints }\end{array}$ & $\begin{array}{l}\text { A simple and low complexity subop- } \\
\text { timal scheme is considered for deter- } \\
\text { mining the water level to allocate the } \\
\text { power for each subcarrier. }\end{array}$ \\
\hline $\begin{array}{l}{[85],} \\
2010\end{array}$ & $\begin{array}{l}\text { Maximize the overall } \\
\text { rate while keeping } \\
\text { the interference ex- } \\
\text { perienced by the PU } \\
\text { pairs below certain } \\
\text { thresholds }\end{array}$ & $\begin{array}{l}\text { Total transmit power } \\
\text { and interference con- } \\
\text { straints }\end{array}$ & $\begin{array}{l}\text { A low-complexity suboptimal power } \\
\text { allocation algorithm is proposed where } \\
\text { CR pairs may use both nonactive and } \\
\text { active PU pair bands as long as the } \\
\text { total cochannel interference and cross- } \\
\text { channel interference do not exceed pre- } \\
\text { scribed limits. }\end{array}$ \\
\hline $\begin{array}{l}{[86],} \\
2010\end{array}$ & $\begin{array}{l}\text { Maximize downlink ca- } \\
\text { pacity while keeping } \\
\text { the interference intro- } \\
\text { duced to the PU band } \\
\text { below a threshold }\end{array}$ & $\begin{array}{l}\text { Total transmit power } \\
\text { and interference con- } \\
\text { straints }\end{array}$ & $\begin{array}{l}\text { A low complexity suboptimal scheme } \\
\text { for joint overlay and underlay power } \\
\text { allocation is presented where equal low } \\
\text { power is allocated to the underlay sub- } \\
\text { carriers and a ladder profile is used to } \\
\text { load power in the overlay subcarriers. }\end{array}$ \\
\hline $\begin{array}{l}{[87],} \\
2009\end{array}$ & $\begin{array}{l}\text { Maximize the weighted } \\
\text { sum rate of SUs in } \\
\text { the downlink, without } \\
\text { causing interferences to } \\
\text { PUs }\end{array}$ & $\begin{array}{l}\text { Total transmit power } \\
\text { and interference con- } \\
\text { straints }\end{array}$ & $\begin{array}{l}\text { A power limited multilevel WF itera- } \\
\text { tive algorithm is considered. }\end{array}$ \\
\hline $\begin{array}{l}{[88],} \\
2007\end{array}$ & $\begin{array}{l}\text { Maximize the capacity } \\
\text { maintaining the PU's } \\
\text { interference limits. }\end{array}$ & $\begin{array}{l}\text { Total transmit power } \\
\text { and per subchannel } \\
\text { power constraints }\end{array}$ & $\begin{array}{l}\text { An iterative partitioned water-filling } \\
\text { algorithm is proposed. }\end{array}$ \\
\hline
\end{tabular}




\subsection{Cognitive Radio System Model and Transmission Power Constraint}

A typical cognitive radio systems is shown in Fig. 3.1 where PUs and SUs share the same bandwidth. In order to avoid harmful interference to each other, the SU needs to detect the opportunities when PUs are not utilizing the spectrum. Higher detection probability without errors provide successful exploitation of opportunities for transmission. In Fig. 3.1, a disk propagation model is considered to illustrate SU transmission. SU can detect any PU's activity within the detection region. However, those PUs that fall outside the detection region (like PU2 in Fig. 3.1), are undetectable by the SU. To deal with this situation, as in [89], PU2 defines a protection region with radius $P$ and needs to maintain a certain interference level $\eta$ within this area. In this scenario, SU's transmission power $P_{\mathrm{tx}}$, subjected to interference constraint, can be written as:

$$
P_{\mathrm{tx}} \leq \eta(D-P)^{\beta}
$$

where $\mathrm{D}$ is the distance between the $\mathrm{SU}$ transmitter and the nearest undetectable PU transmitter, $\beta$ is the path attenuation factor and $\eta$ is the maximum allowable interference level. OFDM is a potential technology in terms of modulation and power control. It is also highly flexible due to reconfigurable subcarrier structure to fit in CRNs for efficient utilization of spectrum opportunities. Fig. 3.2 depicts a typical spectrum structure in OFDM-based CRNs. There are $M$ subchannels licensed to $M$ PU systems that can be potentially used by the SU based on opportunity detection. There are $N$ subcarriers that are distributed among the $M$ subchannels. For example, let the $j^{\text {th }}$ subchannel has total $L_{j}$ subcarriers that can be utilized by the SU when PU is absent. For successful transmission, the SU first needs to test any PU transmitter in the desired subchannel. If found, the sum power of all the subcarriers in that subchannel will be set to zero until the PU transmission ends. If not, the $\mathrm{SU}$ can utilize this subchannel with the interference constraint described in (3.1). Let $G_{j}$ is 


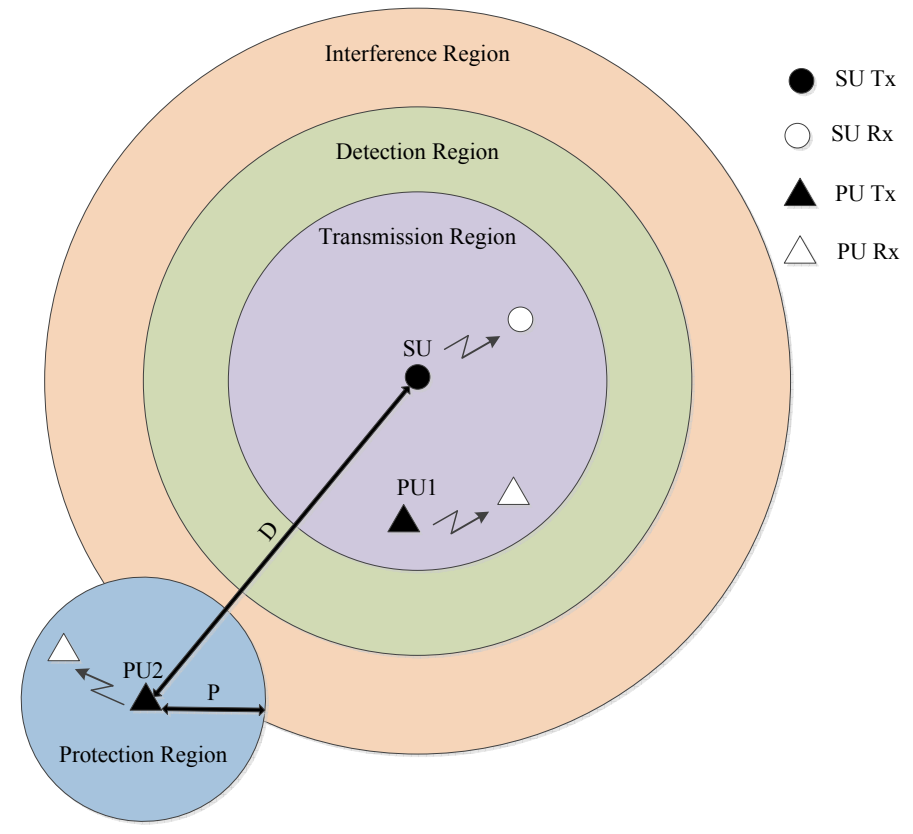

Figure 3.1: Cognitive radio system model.

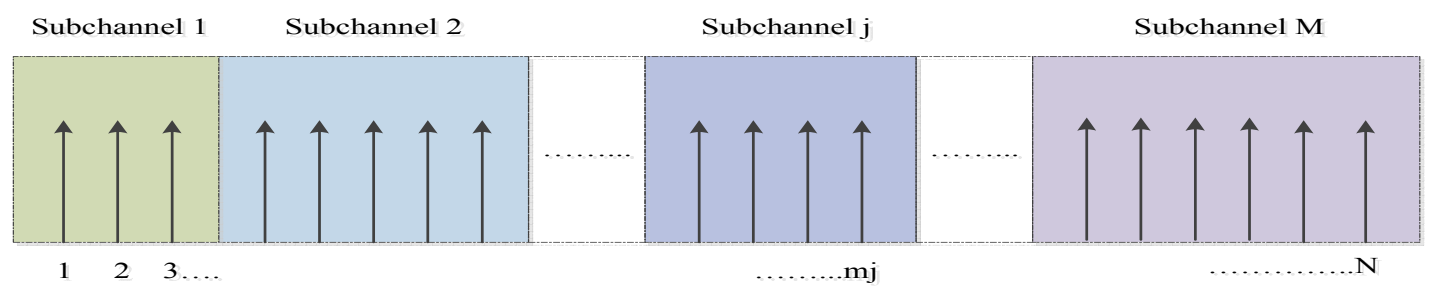

Figure 3.2: Spectrum of SU in OFDM-based CRNs 
the interference constraint on the $j^{\text {th }}$ subchannel after spectrum detection, then,

$$
G_{j} \triangleq \begin{cases}0 & P U_{j} \text { is detected } \\ \eta_{j}\left(D_{j}-P_{j}\right)^{\beta_{j}} & P U_{j} \text { is not detected }\end{cases}
$$

where $\eta_{j}$ is the maximum allowable interference level for $P U_{j}$ within the protection region whose radius is $P_{j}, D_{j}$ is the distance between the SUs transmitter and the nearest undetectable $P U_{j}$ 's transmitter and $\beta_{j}$ is the corresponding path attenuation factor.

\subsection{Problem Formulation}

Consider an OFDM communication system similar to [90]:

$$
y_{n}[m]=h_{n} x_{n}[m]+w_{n}[m], \quad \text { where } n=1, \ldots, N
$$

where $x_{n}[m], y_{n}[m], w_{n}[m]$ and $h_{n}$ are the input, output, noise signal and channel gain, respectively. Assume $P_{T}>0$, as the total power constraint and $P_{i}$ is the transmit power of an OFDM block, then the achievable data rate of reliable communication using the OFDM channel is,

$$
R\left(P_{i}\right)=\sum_{i=1}^{N} \Delta B \log \left(1+\frac{P_{i}\left|h_{i}\right|^{2}}{N_{0}}\right)
$$

where $N_{0}$ is the noise power spectral density and $\Delta \mathrm{B}$ is the subcarrier spacing (bandwidth). Thus the power allocation has to be done to maximize the sum rate in (3.4). In OFDM based CRNs, the subchannel transmit power constraints impose further restrictions on the power allocation in addition to the total transmit power constraint. Assuming the allocated signal power and the propagation path gain for the $i^{\text {th }}$ subcarrier are given as $P_{i}$ and $h_{i}$

(where $\frac{\left|h_{i}\right|^{2}}{N_{0}}=h_{i}$ ) respectively, $i=1, \ldots, N$ and, the weights $w_{i}>0, \forall i$ then the optimal 
power allocation problem can be expressed as:

$$
\begin{gathered}
\max _{\left\{P_{i}\right\}} \sum_{i=1}^{N} w_{i} \log \left(1+h_{i} P_{i}\right) \\
\text { subject to: } \mathrm{C} 1: 0 \leq P_{i} \leq S_{i}, \forall i ; \\
\mathrm{C} 2: \sum_{i=1}^{N} P_{i}=P_{T} \\
\mathrm{C} 3: F_{j} \leq G_{j}, \forall j ;
\end{gathered}
$$

where, $N$ is the total number of subcarriers and $j=1, \ldots, M$; and $M$ is the total number of subchannels, $F_{j}=\sum_{i=m_{j}}^{m_{j+1}-1} P_{i}, j \in A$ is the power allocated to the $j^{\text {th }}$ subchannel and $m_{j}$ is the index of the first subcarrier and $m_{j+1}-1$ is the index of the last subcarrier in the $j^{\text {th }}$ subchannel.

The power allocation problem in (3.5) is constructed considering three constraints. Constraint C1 consider individual peak power constraints, constraint C2 includes total power constraint and constraint C3 incorporates individual subchannel power constraints caused by the PUs's interference limits. Considering constraints $\mathrm{C} 2$ and C3, in [88] the authors proposed IPWF algorithm to obtain the optimal power allocation vector. Constraint C1 was introduced in [78] where the authors proposed GWFPP approach to solve the weighted RRA problems, that also provide optimal result. In this paper, we combined all those constraints to construct an optimal power allocation scheme.

In OFDM based CRNs, the subchannel transmit power constraints impose restrictions on the power allocation in addition to the total transmit power constraint. On the other hand, peak power constraint impose further restriction on the power allocation problem. Thus, a transmission rate constraint associated with the maximum modulation order limitation on each subcarrier is considered. It leads to a peak power constraint on each subcarrier. Since the combining of all three constraints made the power allocation problem more complicated, therefore previous approach cannot be easily utilized to solve the problem, and hence we 
need to develop new approach.

\subsection{Subcarrier Allocation}

Assume $I_{m}$ be the number of subcarriers allocated for one subchannel, is a variable. Since the utilization of any subchannel by the SU, is bounded by the power constraint on the $j^{\text {th }}$ subchannel $\left(G_{j}\right)$, the transmit power for one subchannel on each subcarrier is given by

$$
P_{m, i_{m}}=\frac{G_{j}}{I_{m}}, \quad i_{m} \in \hat{I_{m}}
$$

where $\hat{I_{m}}$ represents the set of subcarriers allocated to one subchannel and $I_{m}$ is the number of subcarriers allocated to one subchannel. The achieveable data rate (using (3.4)) can be shown as

$$
D=\sum_{m=1}^{M} \sum_{i_{m} \in I_{m}} R_{m, i_{m}}\left(P_{m, i_{m}}\right), \quad i_{m} \in \hat{I_{m}}
$$

For a given set $\hat{I_{m}}$ for a subchannel, if one more subcarrier $i_{m}{ }^{*}$ is allocated to that subchannel, i. e. $\hat{I_{m}}$ is replaced by $\hat{I_{m}} \cup\left\{i_{m}{ }^{*}\right\}$, the change of achievable rate for that subchannel, $\Delta d_{m, i_{m}}\left(\hat{I_{m}}\right)$, can be given by

$$
\begin{aligned}
& \Delta d_{m, i_{m}{ }^{*}}\left(\hat{I_{m}}\right)=D_{m}\left(\frac{G_{j}}{I_{m}+1}\right)-D_{m}\left(\frac{G_{j}}{I_{m}}\right) \\
& =\sum_{i_{m} \in \hat{I_{m}} \cup\left\{i_{m^{*}}\right\}} d_{m, i_{m}}\left(\frac{G_{j}}{I_{m}+1}\right)-\sum_{i_{m} \in \hat{I_{m}}} d_{m, i_{m}}\left(\frac{G_{j}}{I_{m}}\right)
\end{aligned}
$$

where $d_{m, i_{m}}$ is the instantaneous achievable rate on the subcarrier $i, \quad i_{m} \in \hat{I_{m}}$.

In our proposed algorithm, assuming $\hat{I_{m}}$ has been allocated to one subchannel, whether an unallocated subcarrier i can be allocated to one subchannel, depends on whether its data rate increase $\Delta d_{m, i_{m}}\left(\hat{I_{m}}\right)$ is the maximum among all the subchannels. That is, subcarrier i will be allocated to $k^{*}=\max _{m, i_{m}} \Delta d_{m, i_{m}}\left(\hat{I_{m}}\right)$. The basics idea behind the subcarrier allocation is well depicted in 3.3 and the algorithm is described in Algorithm 1 description. 


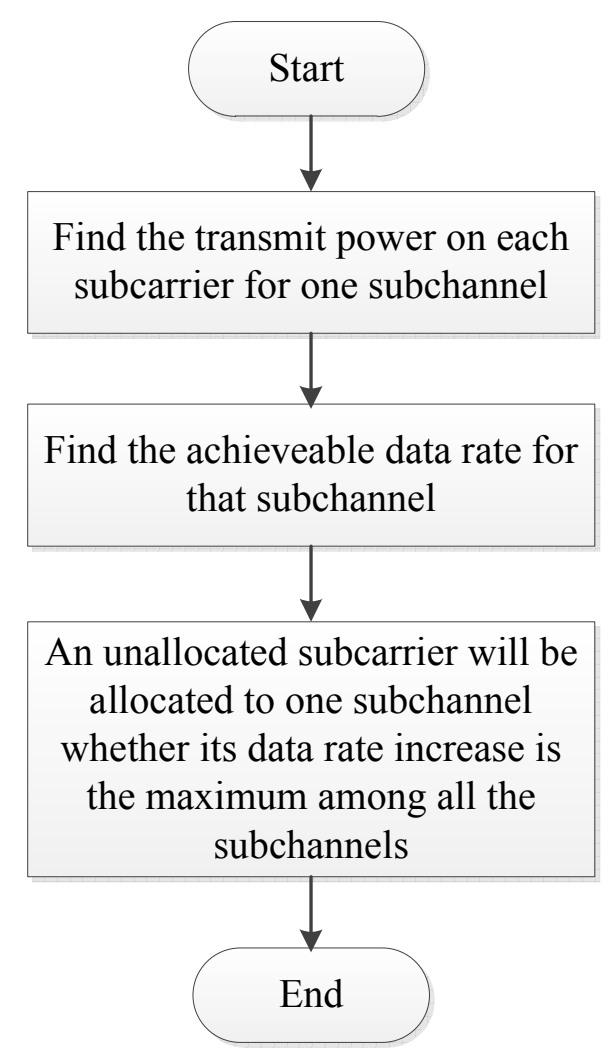

Figure 3.3: Flow chart for subcarrier aliocation.

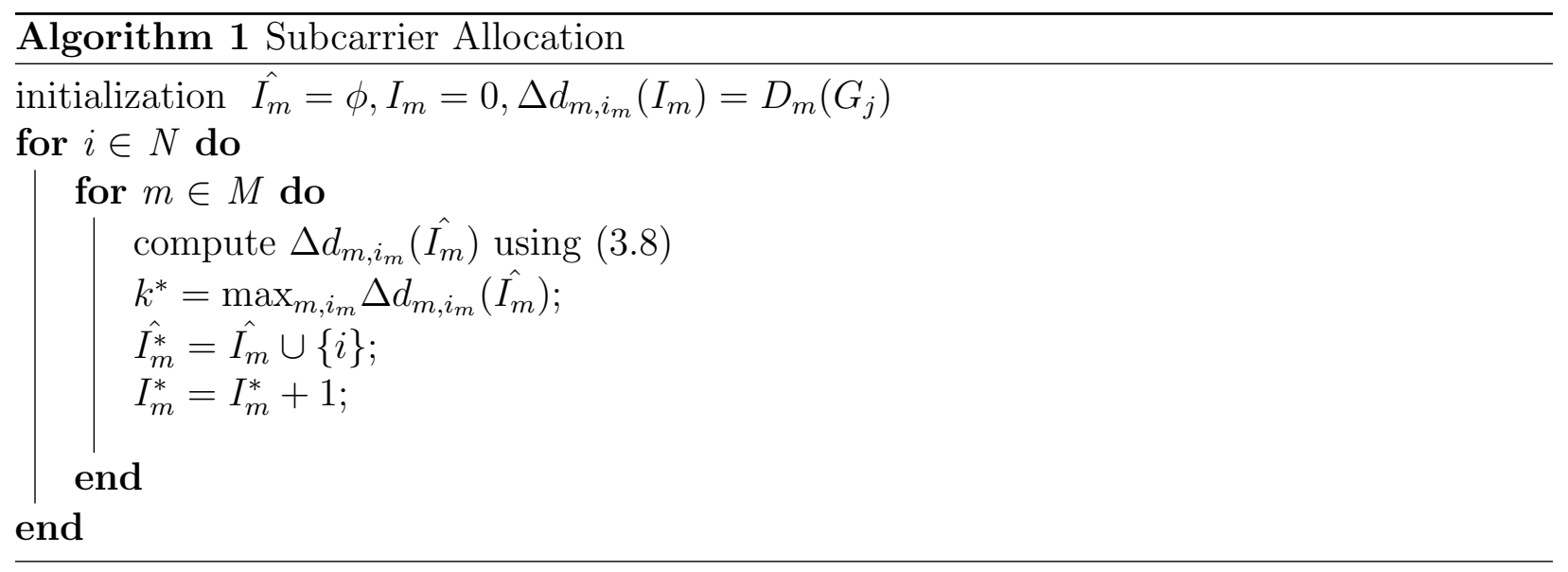


After subcarrier allocation, in the second step, power allocation is carried out on its allocated subcarrier $\hat{A_{m}}$.

\subsection{Power Allocation using GWFPP}

\subsubsection{Iterative Partitioned Weighted Geometric Water-Filling with Individual Peak Power (IGPP)}

In this work, utilizing IPWF [88] and GWFPP [78] approach, we proposed Iterative Partitioned Weighted Geometric Water-Filling with Individual Peak Power (IGPP) algorithm, that also provides optimal result. For IGPP the first step is to divide all the subchannels into two sets, say $\mathrm{A}$ and $\mathrm{B}$, and there are $2^{M}$ partitions in total. $E$ set is a subsequence of the sequence $1,2, \ldots, N$ and $N$ is the total number of subcarriers. The next step would be to perform GWFPP for every $j^{\text {th }}$ subchannel and calculate $P_{i}$ using (??), (4.18) and (4.19). Let the set $\Lambda$ is defined by the set $\left\{i \mid P_{i}>S_{i}, i \in E\right\}$. If $\Lambda$ is an empty set, then $P_{i}$ will be the output, otherwise, $P_{i}$ will be set by the individual peak power $S_{i}$. Everytime we need to update the set $E$ and the total power $P_{T}$. Since the finite set $E$ is getting smaller and smaller until the set $\Lambda$ is empty, algorithm GWFPP carries out at most, $N$ loops to compute the optimal solution [78]. The next steps are to remove partitions that are represented by set $D$ which is a set of subchannels that satisfies $F_{j}<G_{j}$ where $j \in A$. For each of the remaining partitions in the remainder set A, the geometric water-filling is performed on the subcarriers that belong to the $j^{\text {th }}$ subchannel. Algorithm IGPP carries out the loops to compute the optimal solution until the set A is empty. The last step would be to verify each partition whether they satisfy $F_{j}<G_{j}$ where $j \in B$. According to the paper [88], there is only one available partition and the corresponding power allocation vector is the solution. Based on the property of IGPP and the strict constraints on the objective function, there can be only one power allocation vector which satisfies all the conditions. In CWF problem, it is dificult to obtain the explicit expression of the optimal power allocation vector. A derived algorithm 
obtained from [88] and [78] forming IGPP, is described in Algorithm 2 description.

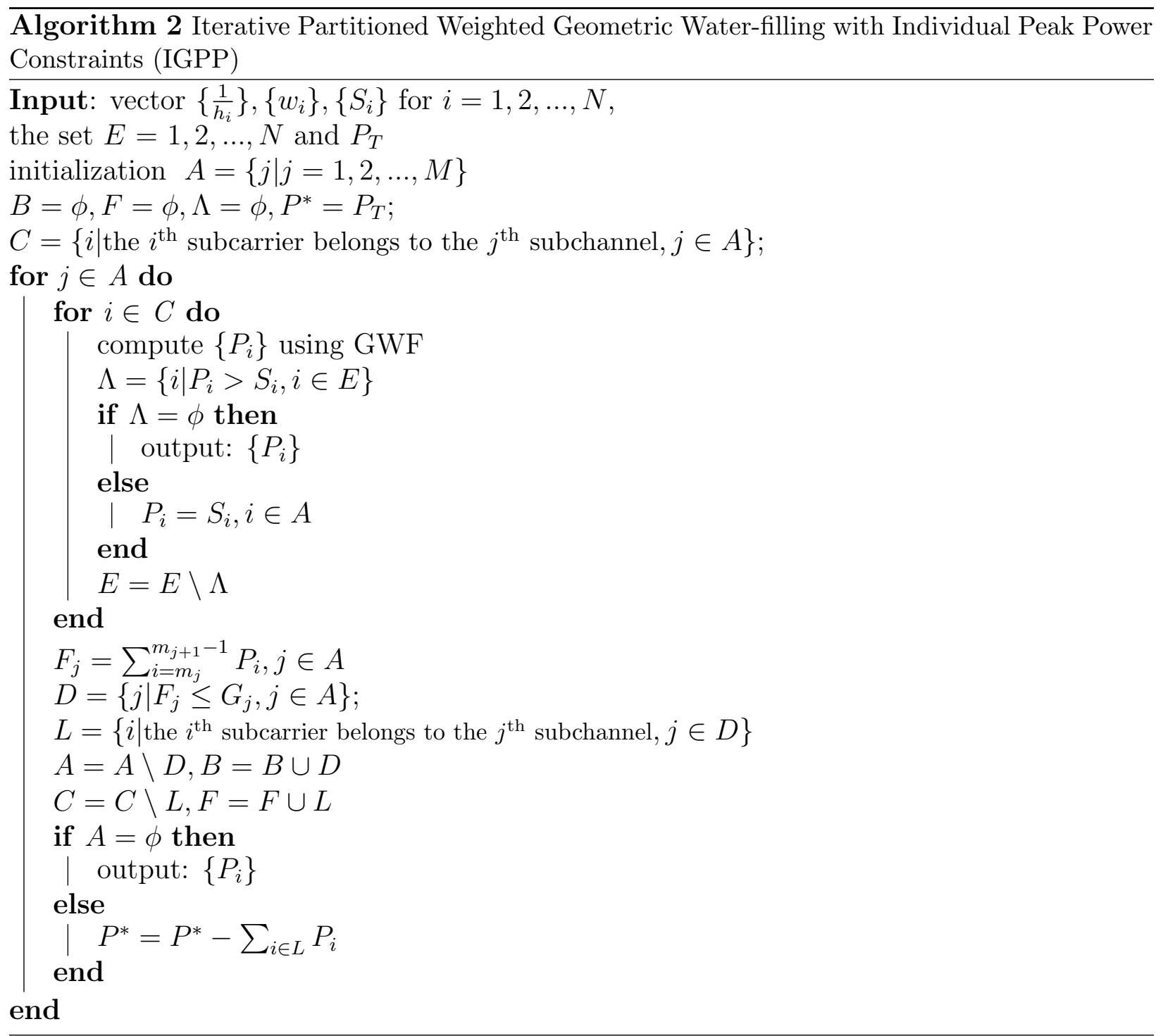

\subsection{Performance Evaluation}

The proposed algorithm IGPP along with the existing methods (IPWF, GWFPP), have been implemented using MATLAB. Randomized fading have been used to generate the outcomes of each algorithms. Here, we assumed that the distance between the $\mathrm{SU}$ and the $\mathrm{PU}$ is 
known, thus equation (3.2) has been used to calculate the subchannel power constraints $\left(G_{j}\right)$. According to the subcarrier assignment strategy, described in previous section, we got subcarriers $1,2,3,4$ to be in subchannel 1 , subcarriers $5,6,7$ to be in subchannel 2 , subcarriers 8, 9, 10, 11 to be in subchannel 3 and the last set of subcarriers $12,13,14,15$ to be in subchannel 4. The simulation parameters are summarized in Table 3.2.

Table 3.2: Simulation Parameters

\begin{tabular}{|c|c|}
\hline Parameters & Values \\
\hline No. of SU and PU & 1,4 \\
\hline Number of subchannels & 4 \\
\hline Number of subcarriers & 15 \\
\hline Protection region $\left(P_{j}\right)$ & $1 \mathrm{~m}$ \\
\hline Path attenuation factor $(\beta)$ & 2 \\
\hline Total power $\left(P_{T}\right)$ & 159 \\
\hline Subcarrier Bandwidth $(\Delta \mathrm{B})$ & $15 \mathrm{KHz}$ \\
\hline Subchannel power constraints $\left(G_{j}\right)$ & $\{72,44,29,17\}$ \\
\hline
\end{tabular}

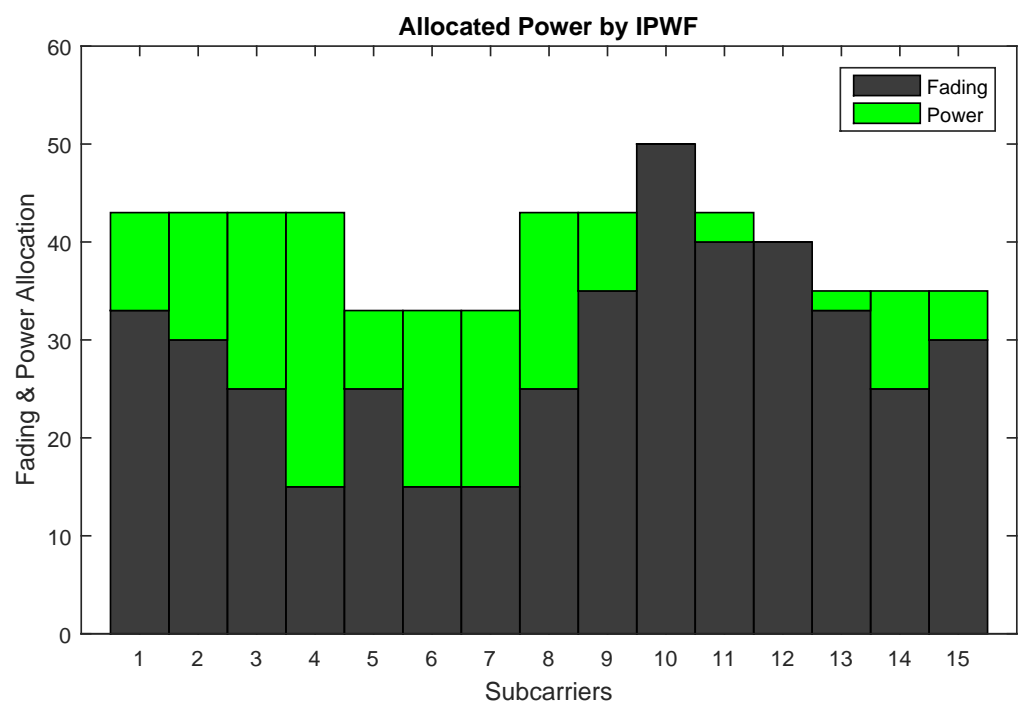

Figure 3.4: Power allocation using Iterative Partitioned Water-Filling Method

Fig. 3.4 shows power allocation when IPWF method has been applied. Since IPWF mainly works based on partitionof sunchannels, the water levels are different for each subchannel due to the total subchannel power constraint for the partitions. 


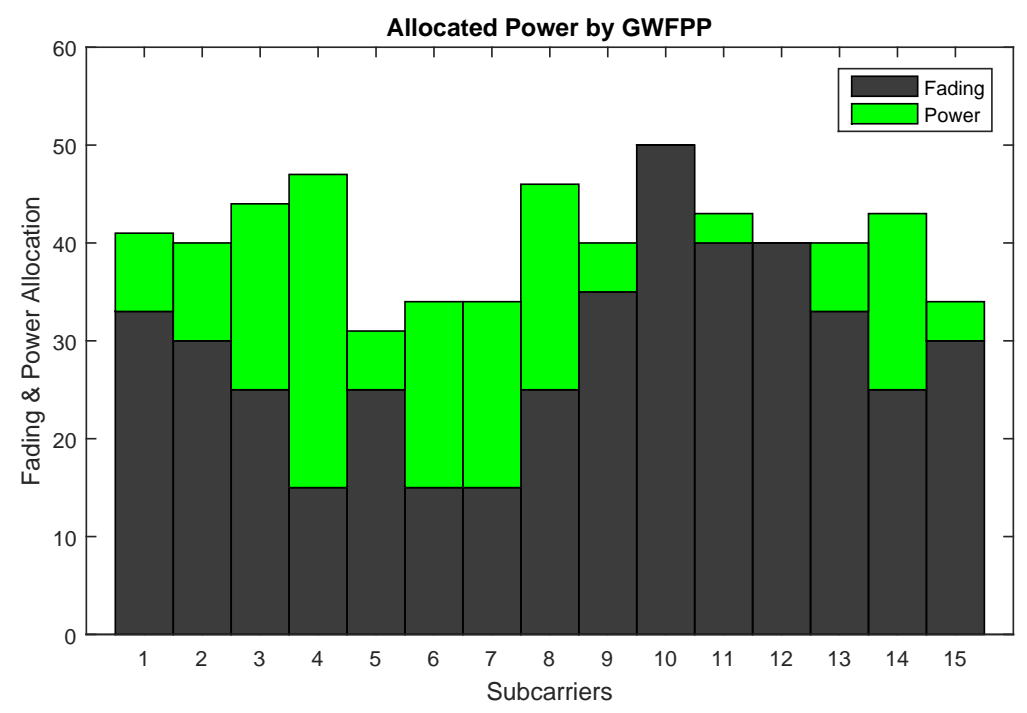

Figure 3.5: Power allocation using Geometric Water-Filling Method with Peak Power Constraints

Fig. 3.5 shows power allocation when GWFPP algorithm has been applied. This algorithm mainly consider individual peak power constraints. As a result, for each channel we get a different set of water level which follows the individual peak power constraints.

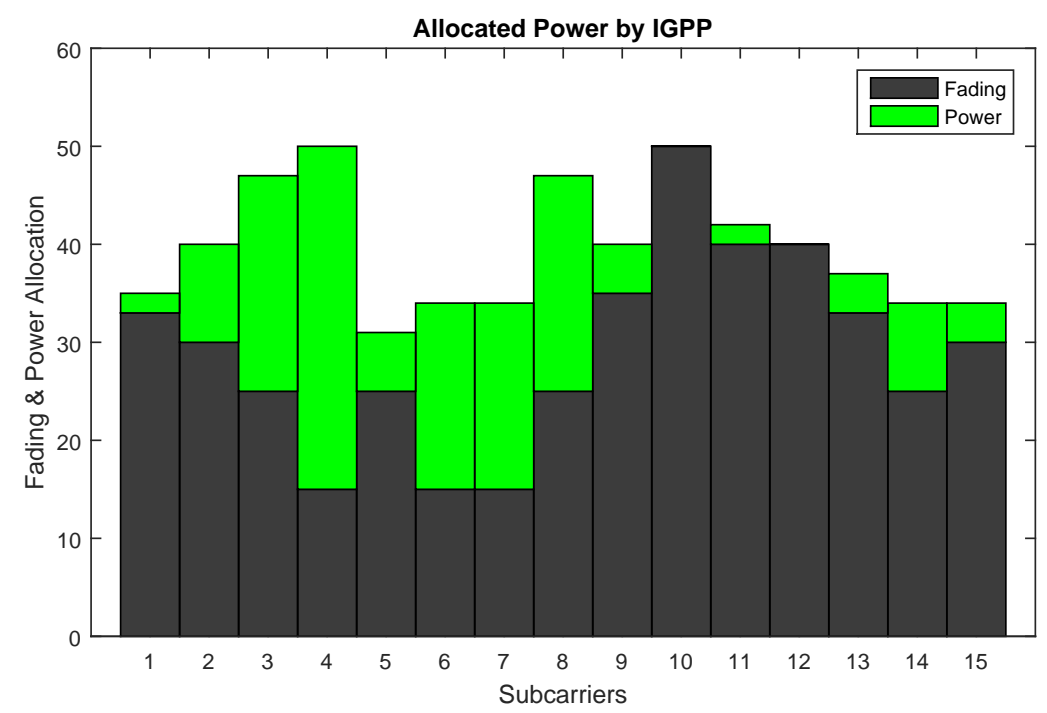

Figure 3.6: Power allocation using Iterative Partitioned Weighted Geometric Water-Filling with Individual Peak Power Constraints Algorithm 
Fig. 3.6 shows power allocation when IGPP algorithm has been applied. Due to the total subchannel power constraint and individual peak power constraints the simulation result varies from the results of IPWF and GWFPP. For each subcarrier we get a different set of water level which follows the individual peak power constraints but they are different from the GWFPP algorithm due to the total subchannel power constraints. Fig. 3.7 shows power allocation by IGPP when considering different weight factors of subcarriers.
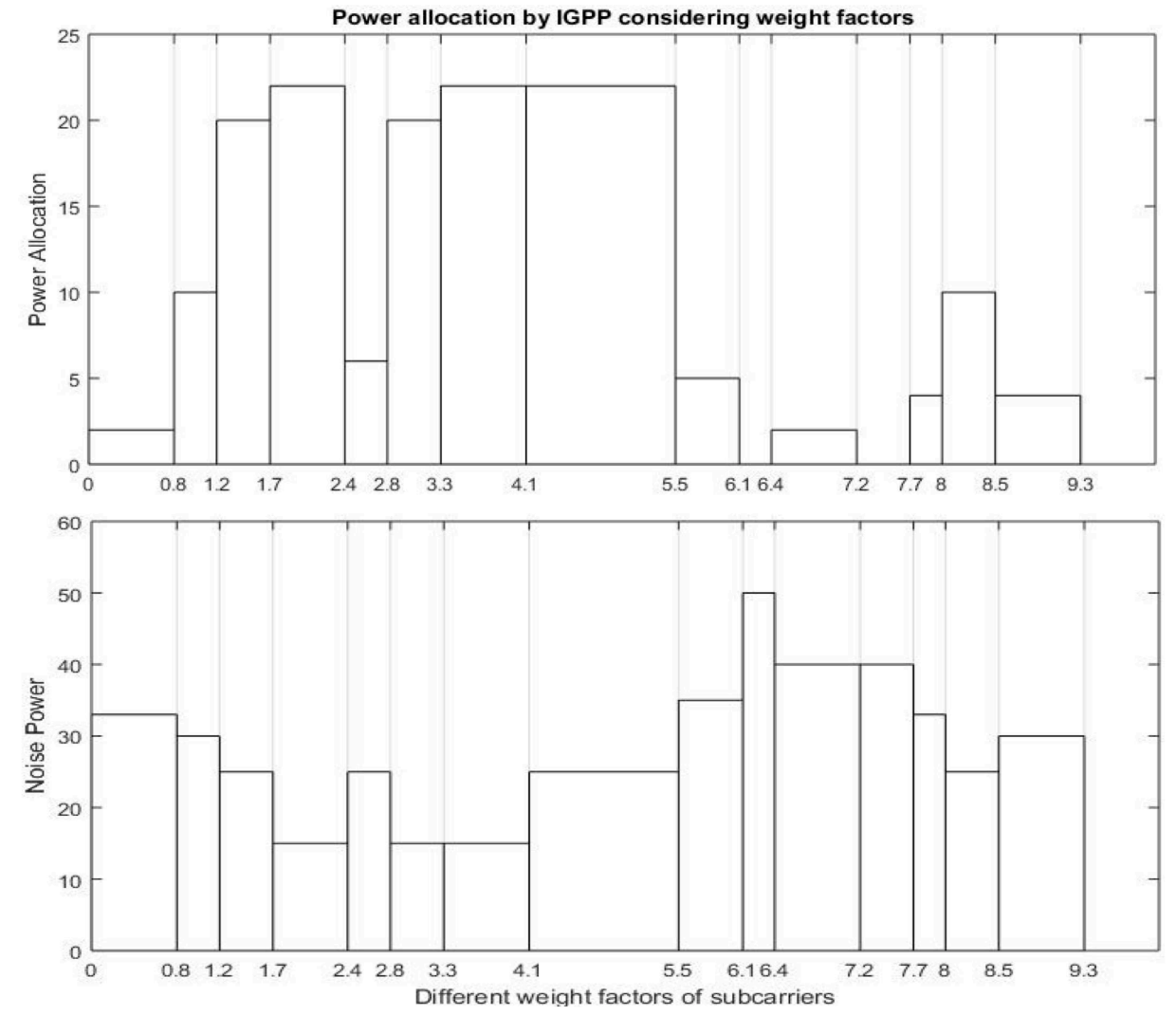

Figure 3.7: Power allocation by IGPP considering weight factors

Fig. 3.7 shows allocated power with corresponding noise power by IGPP when considering different weight factors for subcarriers. In $X$ axis, cumulative summation of weighted coefficients are presented, as we moved towards right. In Fig. 3.7, the width of each bar 
varies due to the different weight factors of the subcarriers.
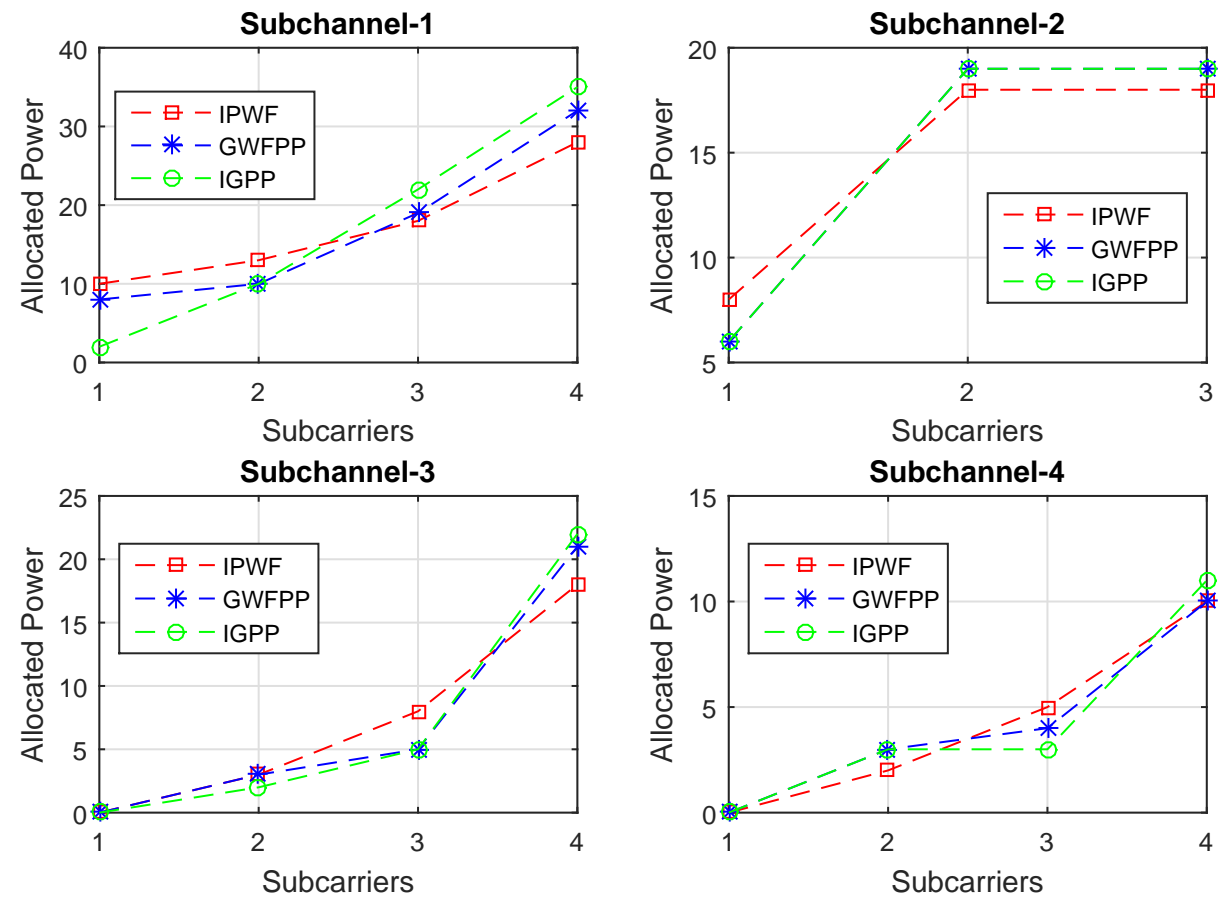

Figure 3.8: Comparison of the sum rates for IPWF, GWFPP and IGPP

Fig. 3.8 compares the allocated power for the IPWF, GWFPP and IGPP algorithms. For each subchannel, we sorted the subcarriers according to their noise power. Thus, the subcarrier that has highest noise power, comes first and the subcarrier that has lowest noise power, comes last for each subchannel. From Fig. 3.8, we found that, for each subchannel, the algorithm IGPP allocated less power in the subcarrier that has highest noise power and allocated more power in the subcarrier that has lowest noise power than the other two algorithms. Thus, the IGPP allows better utilization of the available power resources than IPW and GWFPP.

Fig. 3.9 compares the sum rates for the IPWF, GWFPP and IGPP algorithms. IGPP algorithm allows the sum rate to be better than IPW and GWFPP, due to the better utilisation of the power resources. 


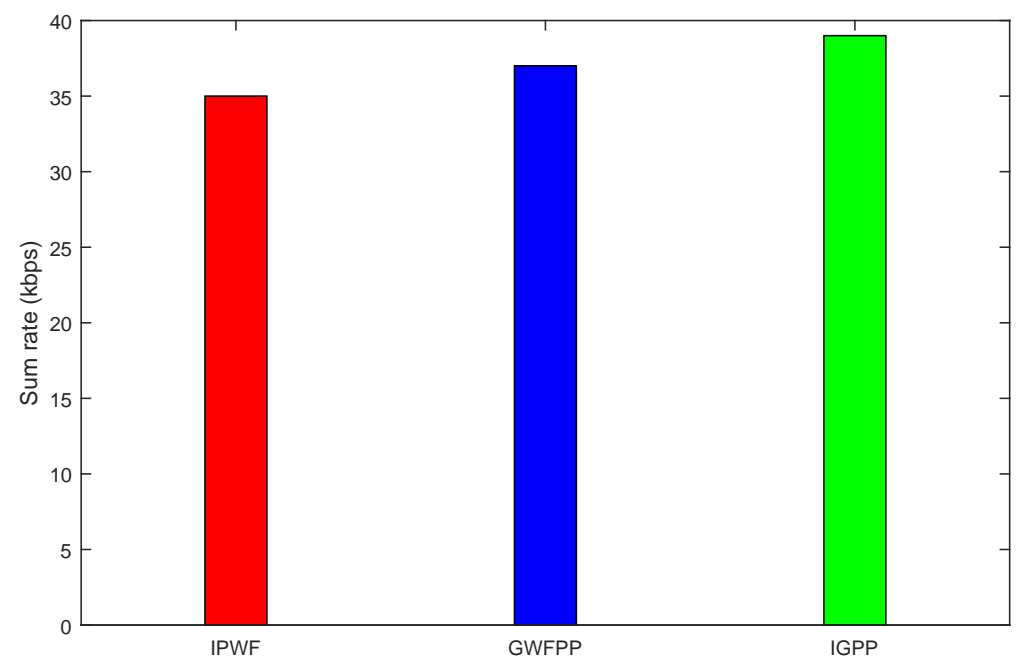

Figure 3.9: Optimal power allocation vs subcarriers for each subchannel using IPWF, GWFPP and IGPP

\subsection{Chapter Summary}

This Chapter provides the system model for OFDM-based CRNs and construct the power allocation problem considering three constraints. Describes the methods for the solution of power allocation problem and presents the proposed algorithms for power allocation along with subcarrier allocation. Also provides comparison of the proposed algorithm results with the related works. 
Chapter 4

Resource Allocation for Cognitive

Radio Systems with D2D

Communication 


\subsection{Introduction}

There have been several burgeoning research efforts found in the literature [91-99] on the RA schemes for CRNs. On the other hand, due to the benefit of D2D communication, International Mobile Telecommunications (IMT)- Advanced Standard systems, such as Long Term Evolution (LTE) and WiMax, allow D2D communication sharing the same radio resources with the cellular network to increase the spectral efficiency [19]. D2D communication is, in fact integrated into LTE-Advanced networks in [100,101]. RRA for D2D communication underlaying cellular networks are currently being extensively investigated by researchers in terms of spectral efficiency [16,102-107] and in terms of energy efficiency $[108,109]$.

From the literature survey, it is found that, most of the recent works focus on how to exploit D2D communication in cellular networks under different constraints. However, only a few efforts $[19,110-114]$ have been made to incorporate D2D communication with CR technology to jointly maximize the spectrum efficiency. In [19], the SUs mode selection strategies are optimized in a cognitive cellular network with D2D communication. A mixed overlay-underlay spectrum sharing method is proposed in [110] for CR-assisted D2D communications in a cellular network. Two spectrum access policies (random and prioritized) are investigated in [111] for cognitive and energy harvesting-based D2D communication in cellular networks. A RA scheme based on CR approach for D2D underlay multicast communication is proposed in [112] to improve system performance. The joint use of full-duplex relay and D2D communication are investigated in [113] for CRNs. An optimal power allocation algorithm is presented in [114] for cognitive D2D communication assisted by two-way relaying.

Table 4.1 provides different approaches to incorporate D2D communication with CR technology.

In this section, the resource allocation problem is studied by jointly employing CR technology and D2D communication in cellular networks. D2D users are modeled as cognitive secondary users where they can opportunistically access the spectrum. An OFDM based 
Table 4.1: Comparative analysis of resource allocation optimization problem in cognitive D2D communication

\begin{tabular}{|c|c|c|c|c|}
\hline Ref. & Objective & Constraint & Solution approach & Performance metric \\
\hline $\begin{array}{l}{[19],} \\
2012\end{array}$ & $\begin{array}{l}\text { Maximize the total } \\
\text { data rates of the } \\
\text { CUs and D2D users }\end{array}$ & $\begin{array}{l}\text { Transmission power bud- } \\
\text { gets and interference con- } \\
\text { straints for the CUs and } \\
\text { D2D users }\end{array}$ & $\begin{array}{l}\text { D2D users optimal power is analyzed } \\
\text { and transmission mode is selected us- } \\
\text { ing the mixed strategy which is up- } \\
\text { dated by replicator dynamics within } \\
\text { the framework of evolutionary game } \\
\text { theory }\end{array}$ & $\begin{array}{l}\text { Replicator dynamics, } \\
\text { population share, } \\
\text { average utilities, } \\
\text { system converge }\end{array}$ \\
\hline $\begin{array}{l}{[110],} \\
2015\end{array}$ & $\begin{array}{l}\text { Maximize the al- } \\
\text { lowable density of } \\
\text { D2D devices }\end{array}$ & $\begin{array}{l}\text { Interference threshold con- } \\
\text { straint, collision probability } \\
\text { constraint }\end{array}$ & $\begin{array}{l}\text { A mixed overlay-underlay spectrum } \\
\text { sharing system is analyzed and a so- } \\
\text { lution is derived to accurately capture } \\
\text { the interactions between devices and } \\
\text { cellular infrastructures using stochas- } \\
\text { tic geometry }\end{array}$ & $\begin{array}{l}\text { Coverage probability, } \\
\text { isolation probability, } \\
\text { interference probabil- } \\
\text { ity }\end{array}$ \\
\hline $\begin{array}{l}{[111],} \\
2015\end{array}$ & $\begin{array}{l}\text { Calculate the over- } \\
\text { all outage probabil- } \\
\text { ity for D2D users }\end{array}$ & $\begin{array}{l}\text { Signal-to-interference-plus- } \\
\text { noise ratio threshold }\end{array}$ & $\begin{array}{l}\text { A complete framework based on } \\
\text { stochastic geometry has been devel- } \\
\text { oped for statistical analysis of cogni- } \\
\text { tive D2D communication using energy } \\
\text { harvested from the ambient interfer- } \\
\text { ence }\end{array}$ & $\begin{array}{l}\text { Transmission } \\
\text { probability, } \\
\text { outage probability }\end{array}$ \\
\hline $\begin{array}{l}{[112],} \\
2014\end{array}$ & $\begin{array}{l}\text { Maximize the to- } \\
\text { tal system capacity } \\
\text { of the PU and SU } \\
\text { multicast group }\end{array}$ & $\begin{array}{l}\text { Transmission power bud- } \\
\text { gets and interference con- } \\
\text { straints for the PUs and } \\
\text { SUs }\end{array}$ & $\begin{array}{l}\text { An optimal channel allocation method } \\
\text { that reduces the interference from cel- } \\
\text { lular to multicast D2D group is pro- } \\
\text { posed }\end{array}$ & $\begin{array}{l}\text { Total capacity of the } \\
\text { system }\end{array}$ \\
\hline $\begin{array}{l}{[113],} \\
2016\end{array}$ & $\begin{array}{lr}\text { Maximize } & \text { the } \\
\text { achievable rate of } \\
\text { D2D links }\end{array}$ & $\begin{array}{l}\text { Signal-to-interference-plus- } \\
\text { noise ratio threshold }\end{array}$ & $\begin{array}{l}\text { D2D communication and full-duplex } \\
\text { relay are utilized in CRN to optimized } \\
\text { the rates of the D2D link }\end{array}$ & Achievable rate gain \\
\hline $\begin{array}{l}{[114],} \\
2013\end{array}$ & $\begin{array}{l}\text { Minimize the SUs } \\
\text { outage probability }\end{array}$ & $\begin{array}{l}\text { Peak transmit power con- } \\
\text { straints, interference power } \\
\text { constraints }\end{array}$ & $\begin{array}{l}\text { An optimal power allocation algorithm } \\
\text { is proposed for a CR network where a } \\
\text { D2D pair as two SUs exchange their } \\
\text { information through a relay node using } \\
\text { network coding }\end{array}$ & Outage probability \\
\hline
\end{tabular}


cognitive cellular network with D2D communication has been designed and analyzed using Lagrange formulation and then solved using geometric water-filling (GWF) method [78]. The optimization problem is characterized by the following five features in order to maximize the transmission rate of the D2D users: a) total power constraint, b) peak power constraint on each subcarrier, c) maximum power constraint of each D2D user, d) interference constraint to the $\mathrm{PU}$ band and e) minimum transmission rate requirements for the D2D users. The senario that we considered to jointly employ CR technology and D2D communication in cellular networks is more practical and yet didn't study by the researchers. For such a cognitive D2D system model, the five sets of constraints are necessary and sufficient in order to maximize the transmission rate of the D2D users. To the best of the authors knowledge, the prior resource allocation researches did not consider the above five features simultaneously for such a cognitive D2D system.

\subsection{System Model}

A single cell downlink OFDM based cognitive cellular system with multiple cellular users as PUs and multiple D2D users as SUs is considered as shown in Fig. 4.1.

In spectral domain, we consider the side-by-side CR access model as shown in Fig. 4.2. It is assumed that the frequency bands with bandwidth $B\left(B_{1}, B_{2}, \ldots, B_{K}\right.$ in $\left.\mathrm{Hz}\right)$, are occupied by $K$ PUs $(1,2, \ldots, K)$. The unoccupied band that can be sensed by the $M$ D2D users $(1$, $2, \ldots, M)$ for possible transmission, is located on both sides of $\mathrm{PU}$ bands. It is possible for the D2D users to opportunistically utilize those unused spectrum by the of knowledge of the environment and cognition capability, to adapt to their radio parameters accordingly [23]. Let, $\mathcal{K}$ be the set for PUs where $\mathcal{K}=\{1,2, \ldots, K\}$ and $\mathcal{M}$ be the set for D2D users where $\mathcal{M}=\{1,2, \ldots, M\}$. The frequency band which is available for CR transmission, is divided into $N$ subcarriers and each subcarrier occupies a bandwidth of $\Delta B \mathrm{~Hz}$.

Each subcarrier transmits in the fading channel where channel gain is integrated by the 


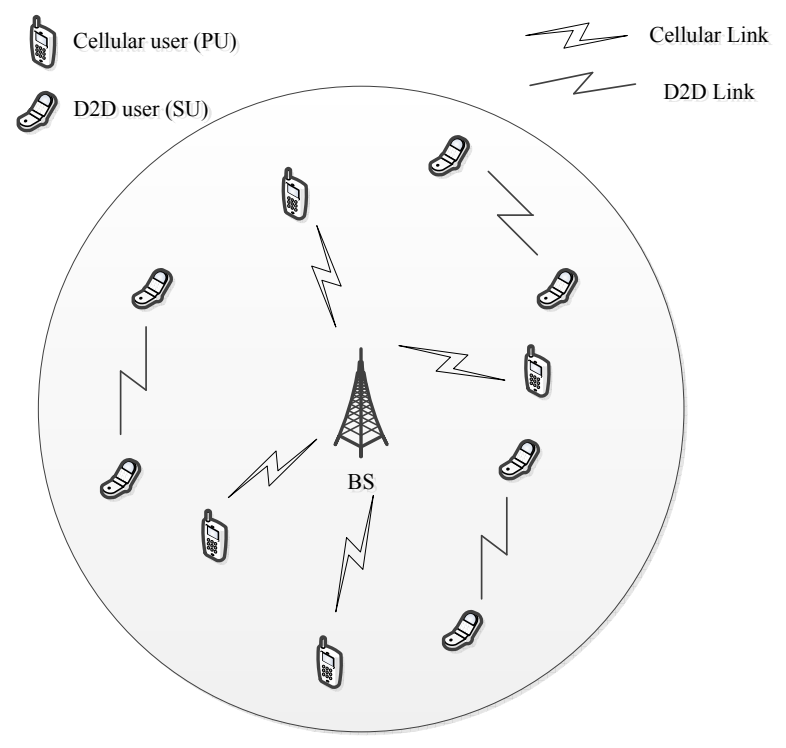

Figure 4.1: A D2D enabled cognitive radio system.

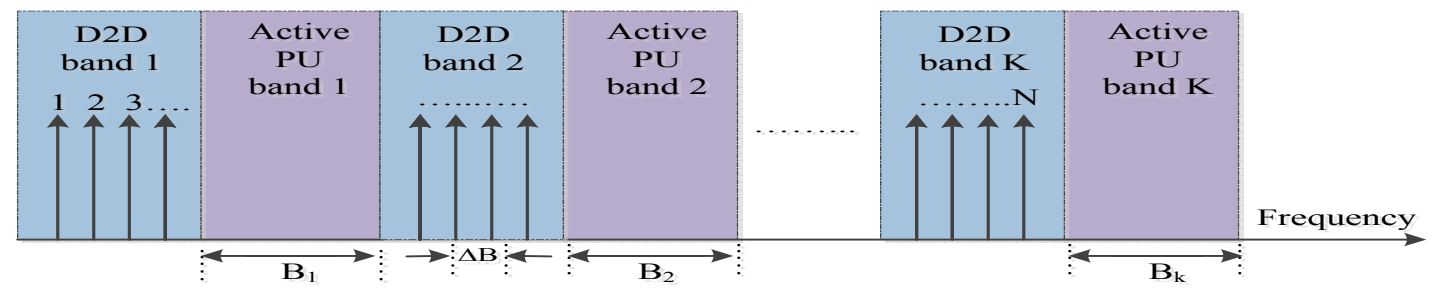

Figure 4.2: Spectrum distribution of PUs and D2D users in OFDM based cognitive D2D system. 
effects of propagation path loss and shadowing. Thus, the D2D users need to detect the channel gain $h_{m, n}^{d d}, h_{m, k}^{d p}$ and $h_{k, m}^{p d}$, by the channel estimation mechanism before transmission. In practice, CSI on the D2D users own channel can be obtained via the classical channel training and feedback techniques. However, the CSI from the D2D user to the PU is not directly available. This can be obtained by the D2D-Tx from the PU-Rx by estimating the reversed channel under the assumption of channel reciprocity. Since our focus is on the resource allocation, we assumed that all the channel estimations are performed using advanced methods, otherwise it will effect the resource allocation scheme. Here, $h_{m, n}^{d d}$ denotes the channel gain between the $m^{\text {th }} \mathrm{D} 2 \mathrm{D}$ user's transmitter and receiver; $h_{m, k}^{d p}$, is the channel gain between the $m^{\text {th }} \mathrm{D} 2 \mathrm{D}$ user's transmitter and the $k^{\text {th }} \mathrm{PU}$ 's receiver; and $h_{k, m}^{p d}$, is the channel gain between the $k^{\text {th }}$ PU's transmitter and the $m^{\text {th }}$ D2D user's receiver. The subscript of index $n$ denotes the $n^{\text {th }}$ subcarrier. Table 4.2 provides a list of frequently used variables and abbreviations.

Due to the coexistence of PU and D2D users in the side-by-side bands, the mutual interference is introduced. Now, the mutual interference introduced by the $n^{\text {th }}$ subcarrier of the $m^{\text {th }}$ D2D user to the $k^{\text {th }} \mathrm{PU}$ band can be written as [115]

$$
\Upsilon_{m, n}^{(k)}=\left|h_{m, k}^{d p}\right|^{2} P_{m, n} T_{s} \int_{d_{m, n}^{(k)}-B_{k} / 2}^{d_{m, n}^{(k)}+B_{k} / 2}\left(\frac{\sin \pi f T_{s}}{\pi f T_{s}}\right)^{2} d f
$$

where, $P_{m, n}$ is the transmit power for the $m^{\text {th }}$ D2D user allocated to the $n^{\text {th }}$ subcarrier, $T_{s}$ is the symbol duration, $d_{m, n}^{(k)}$ represents the spectrum distance between the $n^{\text {th }}$ subcarrier of the $m^{t h}$ D2D user and the $k^{t h} \mathrm{PU}$ band and $B_{k}$ represents occupied bandwidth by the $k^{t h}$ PU.

Let $I_{m, n}^{(k)}=\left|h_{m, k}^{d p}\right|^{2} T_{s} \int_{d_{m, n}-B_{k} / 2}^{d_{m, n}^{(k)}+B_{k} / 2}\left(\frac{\sin \pi f T_{s}}{\pi f T_{s}}\right)^{2} d f$, be the interference factor for the $m^{\text {th }}$ D2D user between the $n^{\text {th }}$ subcarrier and the $k^{\text {th }} \mathrm{PU}$ band. Then equation (4.1) can be rewritten as

$$
\Upsilon_{m, n}^{(k)}=P_{m, n} \cdot I_{m, n}^{(k)}
$$




\section{CHAPTER 4. RESOURCE ALLOCATION FOR COGNITIVE RADIO SYSTEMS WITH D2D COMMUNICATION}

Table 4.2: List of frequently used variables and abbreviations

\begin{tabular}{|c|c|c|}
\hline Group & Variable & Meaning \\
\hline \multirow[t]{5}{*}{ Index } & $k$ & index of PUs, for $k=1,2, \cdots, K$ \\
\hline & $m$ & index of D2D users, for $m=1,2, \cdots, M$ \\
\hline & $l$ & index of other active D2D users, for $l=1,2, \cdots, M$ \\
\hline & $n$ & index of subcarriers, for $n=1,2, \cdots, N$ \\
\hline & $B$ & index of PUs bandwidth $B_{1}, B_{2}, \ldots, B_{K}$ in $\mathrm{Hz}$ \\
\hline \multirow[t]{7}{*}{ Set } & $\mathcal{K}$ & set of PUs $\{1,2, \cdots, K\}$ \\
\hline & $\mathcal{M}$ & set of D2D users $\{1,2, \cdots, M\}$ \\
\hline & $\mathcal{N}$ & set of subcarriers $\{1,2, \cdots, N\}$ \\
\hline & $\mathcal{N}_{m}$ & set of subcarriers allocated to the $m^{t h}$ D2D user $\left\{1,2, \cdots, N_{m}\right\}$ \\
\hline & $\bar{N}_{m}$ & number of subcarriers allocated to the $m^{t h} \mathrm{D} 2 \mathrm{D}$ user \\
\hline & $\lambda$ & set of subcarriers that exceeds the peak power constraint \\
\hline & $\Omega$ & set of subcarriers that need to be reallocated \\
\hline \multirow[t]{7}{*}{ Channel related term } & $h_{m, n}^{d d}$ & channel gain between the $m^{t h}$ D2D user's transmitter and receiver \\
\hline & $h_{m, k}^{d p}$ & $\begin{array}{l}\text { channel gain between the } m^{t h} \text { D2D user's transmitter and the } k^{t h} \\
\text { PU's receiver }\end{array}$ \\
\hline & $h_{k, m}^{p d}$ & $\begin{array}{l}\text { channel gain between the } k^{t h} \text { PU's transmitter and the } m^{t h} \text { D2D } \\
\text { user receiver }\end{array}$ \\
\hline & $\Gamma_{m, n}$ & channel gain-to-noise ratio \\
\hline & $d_{m}$ & link distance between the devices of D2D user \\
\hline & $\eta$ & path attenuation factor \\
\hline & $\sigma_{A W G N}^{2}$ & additive white Gaussian noise variance \\
\hline \multirow[t]{2}{*}{$\begin{array}{l}\text { Bandwidth related } \\
\text { term }\end{array}$} & $d_{m, n}^{(k)}$ & $\begin{array}{l}\text { spectrum distance between the } n^{t h} \text { subcarrier of the } m^{t h} \text { D2D } \\
\text { user and the } k^{t h} \mathrm{PU} \text { band }\end{array}$ \\
\hline & $\Delta B$ & bandwidth of each subcarrier in $\mathrm{Hz}$ \\
\hline \multirow[t]{4}{*}{ Power related term } & $P_{m, n}$ & $\begin{array}{l}\text { transmit power for the } m^{t h} \text { D2D user allocated to the } n^{t h} \text { sub- } \\
\text { carrier }\end{array}$ \\
\hline & $S_{m}$ & total allocated power for the $m^{t h}$ D2D user \\
\hline & $E_{m}$ & extra power for the $m^{t h}$ D2D user \\
\hline & $R_{m}$ & $\begin{array}{l}\text { remaining power for the } m^{t h} \text { D2D user that needs to be reallocated } \\
\text { in the next round }\end{array}$ \\
\hline \multirow[t]{5}{*}{$\begin{array}{l}\text { Interference related } \\
\text { term }\end{array}$} & $\Upsilon_{m, n}^{k}$ & $\begin{array}{l}\text { interference introduced by the } n^{t h} \text { subcarrier of the } m^{t h} \text { D2D user } \\
\text { to the } k^{t h} \mathrm{PU} \text { band }\end{array}$ \\
\hline & $I_{m, n}^{k}$ & $\begin{array}{l}\text { interference factor for the } m^{t h} \text { D2D user between the } n^{t h} \text { subcar- } \\
\text { rier and the } k^{t h} \mathrm{PU} \text { band }\end{array}$ \\
\hline & $\rho_{m, n}^{k}$ & $\begin{array}{l}\text { interference introduced by the } k^{t h} \mathrm{PU} \text { signal to the } m^{t h} \text { D2D user } \\
\text { on the } n^{t h} \text { subcarrier }\end{array}$ \\
\hline & $L_{l, n}$ & interference signal comes from other active D2D users. \\
\hline & $\mathcal{I}_{\text {agg }}$ & aggregated interference \\
\hline \multirow{2}{*}{$\begin{array}{l}\text { Transmission rate re- } \\
\text { lated term }\end{array}$} & $\Re_{m, n}$ & transmission rate for the $m^{t h}$ D2D user on the $n^{t h}$ subcarrier \\
\hline & $\bar{\Re}_{m, n}$ & transmission rate when peak power constraint is considered \\
\hline \multirow[t]{6}{*}{ Constraint Group } & $P_{T}$ & total power constraint \\
\hline & $P_{m, \max }$ & maximum power constraint of the $m^{t h}$ D2D user \\
\hline & $\overline{P_{m, n}}$ & peak power constraint for the $m^{t h}$ D2D user at the $n^{t h}$ subcarrier \\
\hline & $I_{t h}^{k}$ & interference threshold of the $k^{t h}$ PU band \\
\hline & $\Re_{m, \min }$ & minimum transmission rate requirements for the D2D users \\
\hline & \begin{tabular}{l|l}
$x_{m, n}$ \\
\end{tabular} & binary decision variable of subcarrier allocation \\
\hline \multirow[t]{3}{*}{ Others } & $\alpha, \beta, \gamma, \delta, \psi$ & Lagrange multipliers \\
\hline & $\Pi_{m, n}$ & proposed metric for subcarrier allocation \\
\hline & $w_{1}, w_{2}, w_{3}$ & weighting factors for subcarrier allocation \\
\hline \multirow[t]{2}{*}{ Reference Level } & $\mu$ & water level \\
\hline & $j^{*}$ & water level step (highest step under the water) \\
\hline \multirow[t]{3}{*}{ Matrix } & $\mathbf{X}$ & $M \times N$ matrix of subcarrier allocation \\
\hline & $\mathbf{P}$ & $M \times N$ matrix of allocated power \\
\hline & $\mathbf{T}$ & $\begin{array}{l}M \times N \text { matrix of allocated power when binary decision variable } \\
\text { is considered }\end{array}$ \\
\hline \multirow[t]{5}{*}{ Abbreviations } & PSD & power spectrum density \\
\hline & ASA & Adaptive subcarrier allocation \\
\hline & PA & Power allocatign \\
\hline & GWF & Geometric water-filling \\
\hline & GWFPP & Geometric water-filling with peak power constraint \\
\hline
\end{tabular}


Now, with an ideal coding scheme, the transmission rate for the $m^{\text {th }} \mathrm{D} 2 \mathrm{D}$ user on the $n^{\text {th }}$ subcarrier, is given by the following formula

$$
\Re_{m, n}\left(P_{m, n}, d_{m}\right)=\Delta B \log \left(1+\frac{P_{m, n}\left|h_{m, n}^{d d}\right|^{2} d_{m}^{-\eta}}{\mathcal{I}_{\text {agg }}}\right)
$$

where, $d_{m}$ represents the link distance between the devices of D2D user and $\eta$ is the path attenuation factor. In (4.3), $\mathcal{I}_{\text {agg }}=\sigma_{A W G N}^{2}+\sum_{k=1}^{K} \rho_{m, n}^{k}+\sum_{l=1, l \neq m}^{M} L_{l, n}$ denotes the aggregated interference, where $\sigma_{A W G N}^{2}$ denotes the additive white Gaussian noise (AWGN) variance, the second term be the interference introduced by the $k^{\text {th }} \mathrm{PU}$ signal to the $m^{\text {th }}$ D2D user on the $n^{\text {th }}$ subcarrier and the third term represents the interference signal comes from other active D2D users.

Let $\Gamma_{m, n}=\frac{\left|h_{m, n}^{d d}\right|^{2} d_{m}^{-\eta}}{\mathcal{I}_{a g g}}$ represents the channel gain-to-noise ratio, then (4.3) can be rewritten as

$$
\Re_{m, n}\left(P_{m, n}, d_{m}\right)=\Delta B \log \left(1+P_{m, n} \Gamma_{m, n}\right) .
$$

In this paper, a transmission rate constraint associated with the maximum modulation order limitation on each subcarrier is considered. It leads to a peak power constraint $\left(\bar{P}_{m, n}\right)$ for the $m^{\text {th }}$ D2D user on the $n^{\text {th }}$ subcarrier which can be written from (4.4) as

$$
\bar{P}_{m, n}=\frac{2^{\bar{\Re}_{m, n}}-1}{\Gamma_{m, n}}
$$

where $\bar{\Re}_{m, n}$ is the corresponding transmission rate when $\bar{P}_{m, n}$ is considered.

Let $x_{m, n}$ be a binary decision variable of channel (subcarrier) allocation. If subcarrier $n$ is allocated to D2D user $m$, then $x_{m, n}$ is equal to 1 ; otherwise it is equal to zero. Since each subcarrier is exclusively allocated to one D2D user, then

$$
\sum_{m=1}^{M} x_{m, n}=1, \quad n=1,2, \ldots, N .
$$


Now, the total system transmission rate is given by

$$
\Re(\mathbf{X}, \mathbf{P})=\Delta B \sum_{m=1}^{M} \sum_{n=1}^{N} x_{m, n} \log \left(1+P_{m, n} \Gamma_{m, n}\right)
$$

where, $\mathbf{X}$ is a $M \times N$ matrix of subcarrier allocation indices $x_{m, n}$, and $\mathbf{P}$ is a $M \times N$ matrix of allocated power $P_{m, n}$.

\subsection{Problem Formulation}

Our objective of RA (subcarrier and power allocation) is to maximize the total downlink transmission rate of the D2D system under both power and interference constraints along with the minimum rate requirements. Therefore, the optimization problem can be formulated as follows

$$
\max _{\mathbf{X}, \mathbf{P}} \Delta B \sum_{m=1}^{M} \sum_{n=1}^{N} x_{m, n} \log \left(1+P_{m, n} \Gamma_{m, n}\right)
$$

subject to:

$$
\begin{aligned}
C 1: & \sum_{m=1}^{M} x_{m, n}=1, x_{m, n} \in\{0,1\} ; \forall n \in \mathcal{N} \\
C 2: & \sum_{m=1}^{M} \sum_{n=1}^{N} x_{m, n} P_{m, n} \leq P_{T}, P_{m, n} \geq 0 ; \\
C 3: & \sum_{n=1}^{N} x_{m, n} P_{m, n} \leq P_{m, \max } ; \forall m \in \mathcal{M} \\
C 4: & x_{m, n} P_{m, n} \leq \bar{P}_{m, n} ; \forall m \in \mathcal{M}, \forall n \in \mathcal{N} \\
C 5: & \sum_{k=1}^{K} \sum_{n=1}^{N} x_{m, n} \Upsilon_{m, n}^{(k)} \leq I_{t h} ; \forall m \in \mathcal{M} \\
C 6: & \sum_{n=1}^{N} x_{m, n} \Re_{m, n} \geq \Re_{m, m i n} ; \forall m \in \mathcal{M}
\end{aligned}
$$


where, constraint $\mathrm{C} 1$ depicts that each subcarrier can not be reused by more than one D2D user. Constraint C2, C3 and C4 denote total power constraint (e. g. $P_{T}$ ), maximum power constraint of each D2D user (e. g. $\left.P_{m, \max }\right)$, and peak power constraint on each subcarrier (e. g. $\left.\bar{P}_{m, n}\right)$ respectively. Constraint C5 describes interference constraint where $I_{t h}$ denotes the total interference threshold by the $K$ PU bands. Lastly, constraint C6 provides minimum transmission rate requirements for the D2D users.

The optimization problem in (4.8) is nonconvex due to binary decision variable $x_{m, n}$. This variable can be relaxed by applying a time sharing approach [116] to allow any value in the interval $(0,1]$ instead of $\{0,1\}$ set, which is equivalent to allowing time-sharing of a single subcarrier between different users. By time-sharing we mean that several users can transmit on a given subcarrier during a given scheduling interval, with each user transmitting alone for a fraction of the interval. This corresponds to a sort of time division multiple access subdivision of the scheduling time unit and is not to be confused with overlapping transmission. Let $t_{m, n}=x_{m, n} P_{m, n}$, then the optimization problem in (4.8) can be rewritten 
as follows:

$$
\max _{\mathbf{X}, \mathbf{T}} \Re(\mathbf{X}, \mathbf{T})=\Delta B \sum_{m=1}^{M} \sum_{n=1}^{N} x_{m, n} \log \left(1+\frac{t_{m, n}}{x_{m, n}} \Gamma_{m, n}\right)
$$

subject to:

$$
\begin{aligned}
C 1: & \sum_{m=1}^{M} x_{m, n}=1, x_{m, n} \in(0,1] ; \quad \forall n \in \mathcal{N} \\
C 2: & \sum_{m=1}^{M} \sum_{n=1}^{N} t_{m, n} \leq P_{T}, t_{m, n} \geq 0 ; \\
C 3: & \sum_{n=1}^{N} t_{m, n} \leq P_{m, \max }, \quad \forall m \in \mathcal{M} \\
C 4: & t_{m, n} \leq \bar{P}_{m, n} ; \quad \forall m \in \mathcal{M}, \quad \forall n \in \mathcal{N} \\
C 5: & \sum_{k=1}^{K} \sum_{n=1}^{N} t_{m, n} I_{m, n}^{(k)} \leq I_{t h} ; \quad \forall m \in \mathcal{M} \\
C 6: & \sum_{n=1}^{N} x_{m, n} \Re_{m, n} \geq \Re_{m, \min } ; \quad \forall m \in \mathcal{M}
\end{aligned}
$$

The problem in (4.9) is convex with a concave objective function which is equivalent to the original problem in (4.8) when the condition on $x_{m, n}$ is relaxed for each D2D user $m$ and subcarrier $n$. Finding the optimal pair $\left(x_{m, n}^{*}, t_{m, n}^{*}\right)=\left(x_{m, n}^{*}, x_{m, n}^{*} P_{m, n}^{*}\right)$ leads to the same solution as finding $\left(x_{m, n}^{*}, P_{m, n}^{*}\right)$.

The analytical solution of the problem in (4.9) is

$$
P_{m, n}^{*}=\left[\frac{1+\psi_{m}}{\ln \left(\alpha_{n}+\beta_{m}+\gamma_{n}+\sum_{k=1}^{K} \delta_{k} I_{m, n}^{(k)}\right)}-\frac{1}{\Gamma_{m, n}}\right]^{+}
$$

Proof: See Appendix

The $P_{m, n}^{*}$ denotes the optimal power allocation for the $m^{\text {th }}$ D2D user on the $n^{t h}$ subcarrier 
when $m=m^{*}$. Thus, the indices of the subcarrier allocation matrix $X$ will be

$$
x_{m, n}= \begin{cases}1 & m=m^{*} \text { for } n=1,2, \ldots, N \\ 0, & \text { otherwise }\end{cases}
$$

The power allocation strategy in (4.10) is indeed corresponding to the CWF strategy [76] and depends on the Lagrange multipliers $(\alpha, \beta, \gamma, \delta, \psi)$ associated with the per user power and interference constraints. By using the CWF, the optimal power allocation strategy can be calculated which is practically intractable. Thus, in this paper, GWF approach [78] is utilized to solve the CWF problem which is described in the following section. It has two advantages:

1. The geometric approach can compute the exact solution to the CWF, including the weighted case, with less computation without determining the water level through solving the non-linear system,

2. Machinery of the proposed geometric approach can overcome the limitations of the CWF algorithm to include more stringent constraints.

In terms of complexity, the CWF approach has a worst-case complexity of $\mathrm{N}$ iterations, i.e., total $O\left(N^{2}\right)$ fundamental arithmetic and logical operations under the $2(N+1)$ memory requirement. On the other hand, GWF algorithm occupies less computational resource. The worst-case computational complexity of the GWF algorithm is $8 N+3$ fundamental arithmetic and logical operations under the $2(N+1)$ memory requirement and for GWFPP, the worst-case complexity is $4 N^{2}+7 N$ fundamental arithmetic and logical operations.

\subsection{Proposed Resource Allocation Scheme}

Based on the analysis in the previous section, an efficient RA scheme is proposed in this section to address the problem defined in (4.8). A two-stage approach is considered to 
solve (4.8) efficiently. Specifically, the resource allocation scheme is divided into two individual procedures: adaptive subcarrier allocation (ASA) and power allocation (PA). In the first stage, each subcarrier is assigned to one D2D user with the minimum value of the proposed metric that is adaptive in nature. In the second stage, power is allocated among all the D2D users to maximize the transmission rate. The procedure for the RA scheme is illustrated in Fig. 4.3.

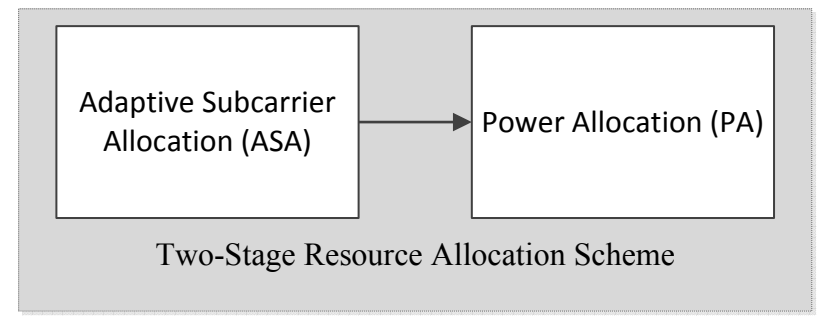

Figure 4.3: The procedure for resource allocation scheme.

\subsubsection{Adaptive Subcarrier Allocation (ASA)}

Most of the recent works regarding subcarrier allocation strategy to the D2D users is based on greedy approach $[104,105]$. In order to assign subcarriers to the D2D users, a proposed metric is utilized that is adaptive in nature. The proposed metric is related to the amount of power, interference and transmission rate.

Let $\mathcal{N}$ be the set of subcarriers where $\mathcal{N}=\{1,2, \ldots, N\}, \mathcal{N}_{m}$ be the set of subcarriers allocated to the $m^{\text {th }}$ D2D user and $\bar{N}_{m}$ denotes the number of subcarriers allocated to the $m^{\text {th }}$ D2D user. Now, the transmission rate increment for one more subcarrier allocation to the $m^{\text {th }}$ D2D user can be written as

$$
\Delta \Re_{m, n}=\Re_{m, n}\left(\frac{P_{m, n}}{\bar{N}_{m}+1}, d_{m}\right)-\Re_{m, n}\left(\frac{P_{m, n}}{\bar{N}_{m}}, d_{m}\right), n \in \mathcal{N}_{m}
$$

The power increment for transmitting one more increment in transmission rate $\Delta \Re$ on 
subcarrier $n$ of the $m^{\text {th }}$ D2D user can be written as

$$
\Delta P_{m, n}=\frac{\left(2^{\Re_{m, n}+\Delta \Re_{m, n}}-2^{\Re_{m, n}}\right)}{\Gamma_{m, n}} .
$$

The interference increment caused by subcarrier $n$ of the $m^{\text {th }} \mathrm{D} 2 \mathrm{D}$ user for the $k^{\text {th }} \mathrm{PU}$ can be written as

$$
\Delta \Upsilon_{m, n}^{(k)}=\Delta P_{m, n} \cdot I_{m, n}^{(k)}
$$

A metric composed of three parts for the $n^{\text {th }}$ subcarrier of the $m^{\text {th }} \mathrm{D} 2 \mathrm{D}$ user is described as

$$
\Pi_{m, n}=w_{1}\left(\frac{\Delta P_{m, n}}{P_{m, \text { max }}-P_{m, \text { used }}}\right)+w_{2}\left(\sum_{k=1}^{K} \frac{\Delta \Upsilon_{m, n}^{k}}{I_{\text {th }}-\Upsilon_{m, \text { used }}^{k}}\right)+w_{3}\left(\frac{\Delta \Re_{m, n}}{\Re_{m, \text { used }}-\Re_{m, \text { min }}}\right)
$$

where, $P_{m, \text { used }}$ is the amount of power that has been utilized for the $m^{\text {th }} \mathrm{D} 2 \mathrm{D}$ user, $\Upsilon_{m, \text { used }}^{k}$ is the interference that has been initiated to the $k^{t h} \mathrm{PU}$, and $\Re_{m, \text { used }}$ is the corresponding transmission rate of the $m^{t h}$ D2D user. The first part consists of the amount of the power increment for transmitting one more increment in transmission rate and the amount of the unused power. The second part includes the amount of interference that is used for considering the interference constraints. Finally, the third part is associated with the minimum transmission rate requirements among the D2D users. Three non-negative parameters $\left(w_{1}, w_{2}, w_{3}\right)$ are exploited as the weighting factors to make a trade-off between the power and the interference constraints together with the minimum rate requirements in the subcarrier allocation process. This is the reason why this subcarrier assignment strategy is named as adaptive subcarrier allocation (ASA).

For a specific D2D user, a subcarrier $n^{*}$ with the minimum value of the metric (4.15), is assigned to that selected D2D user $m^{*}$. A subcarrier with a lower value depicts that the power and interference increments for providing one more increment in transmission rate are relatively small compared to other subcarriers. The ASA strategy is well described in 
Algorithm 1.

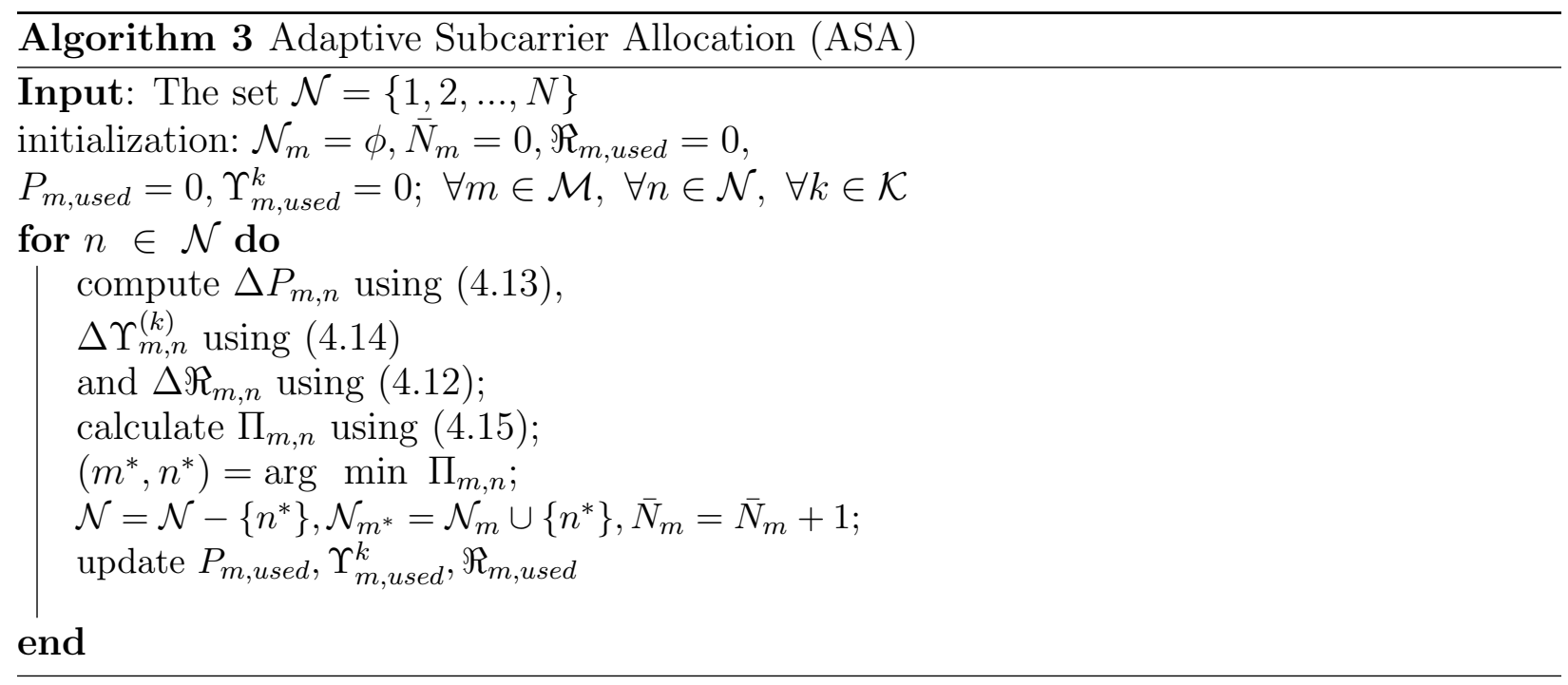

\subsubsection{Power Allocation (PA)}

After subcarrier assignment described in the previous section, power allocation is performed for the selected subcarrier $n^{*}$ of the D2D user $m^{*}$. For the selected subcarrier $n^{*}$ of the D2D user $m^{*}$, the value of $x_{m^{*}, n^{*}}$ becomes 1 and then the power allocation problem in (4.8) can be solved using GWF approach. Fig. 4 gives an illustration of the GWF approach.

Instead of trying to determine the common water level $\mu$ (real nonnegative number) in CWF, the highest water level step, denoted by $j^{*}$, which is an integer number, is introduced to find the solutions for power allocation. Let $P(j)$ denotes the water volume above step $j$ or zero, whichever is greater and the value of $P(j)$ can be found as:

$$
P(j)=\left[P_{T}-\left\{\sum_{n=1}^{j-1}\left(\frac{1}{\Gamma_{m, j}}-\frac{1}{\Gamma_{m, n}}\right)\right\}\right]^{+} ; \forall j \in \mathcal{N}_{m}
$$

where $\frac{1}{\Gamma_{m, n}}$ is the "step depth" of the $n^{\text {th }}$ stair. Due to the definition of $P(j)$ being the power (water volume) above step $j$, it cannot be a negative number. Therefore we use $\{.\}^{+}$ in (4.16) to assign 0 to $P(j)$ if the result inside the bracket is negative. The corresponding 


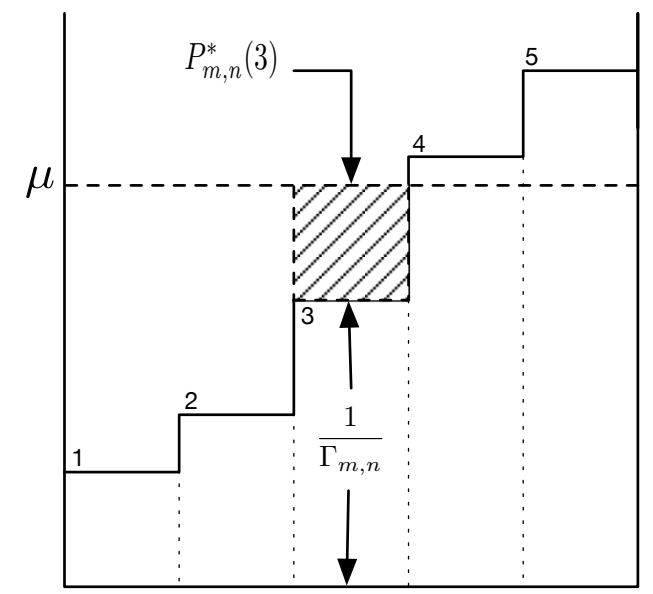

(a)

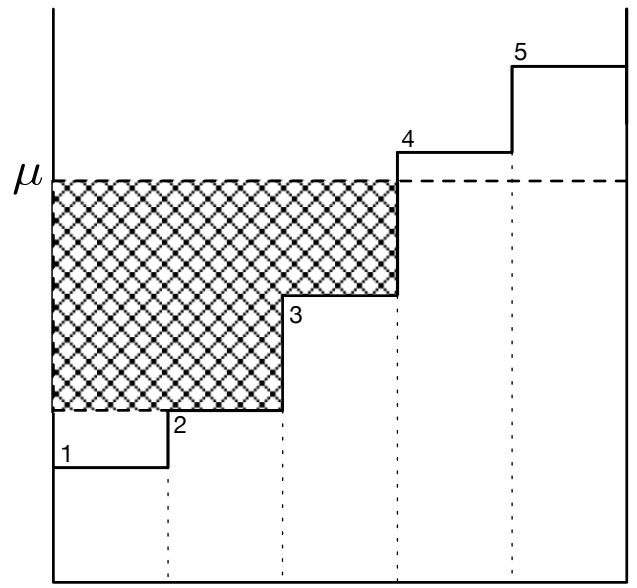

(b)

Figure 4.4: Illustration for the GWF algorithm (a) Illustration of water level step $j^{*}=3$, allocated power for the third step $P_{m, n}^{*}(3)$, and step depth is $\frac{1}{\Gamma_{m, n}}$ and (b) Illustration of $P(j)$ (shadowed area, representing the total power above step $j$ ) when $j=2$.

geometric meaning is that the $j^{*^{t h}}$ level is above water.

According to [78], the explicit solution for power allocation is:

$$
P_{m, n}= \begin{cases}P_{m, j^{*}}+\left(\frac{1}{\Gamma_{m, j^{*}}}-\frac{1}{\Gamma_{m, n}}\right) & 1 \leq n \leq j^{*} \\ 0, & j^{*}<n \leq N_{m}\end{cases}
$$

where the water level step $j^{*}$ is given as

$$
j^{*}=\max \left\{j \mid P(j)>0,1 \leq j \leq N_{m}\right\}
$$

and the power level for this step is

$$
P_{m, j^{*}}=\frac{1}{j^{*}} P\left(j^{*}\right)
$$

In order to allocate power to the selected subcarriers for each D2D user, first we perform geometric water-filling with peak power constraint (GWFPP) [78] for all the subcarriers and 
calculate $\left\{P_{m, n}\right\}$ using (4.17), (4.18) and (4.19). Now, let $\lambda$ is defined by the set $\left\{n \mid P_{m, n}>\right.$ $\left.\bar{P}_{m, n}, n \in \mathcal{N}_{m}\right\}$. If $\lambda$ is an empty set, then $P_{m, n}$ will be the output, otherwise, $P_{m, n}$ will be set by the individual peak power $\bar{P}_{m, n}$. Everytime we need to update the set $\mathcal{N}_{m}$ and the total power $P_{T}$ at the end of each iteration. Since the finite set $\mathcal{N}_{m}$ is getting smaller and smaller until the set $\lambda$ is empty. The required steps to apply the algorithm GWFPP is summarized below:

\section{Algorithm GWFPP}

Input: vector $\left\{\frac{1}{\Gamma_{m, n}}\right\},\left\{\bar{P}_{m, n}\right\} \forall n \in \mathcal{N}_{m}$ and $P_{T}$.

1) Compute $\left\{P_{m, n}\right\}$ using (4.17), (4.18) and (4.19).

2) The set $\lambda$ is defined by the set $\left\{n \mid P_{m, n}>\bar{P}_{m, n}, n \in \mathcal{N}_{m}\right\}$. If $\lambda$ is the empty set, output $\left\{P_{m, n}\right\}_{n=1}^{N_{m}}$; else, $P_{m, n}=\bar{P}_{m, n}$, as $n \in \lambda$.

3) Update $\mathcal{N}_{m}$ and $P_{T}$. Then return to 1) of the GWFPP.

The next step is to compute the summation of allocated power for all D2D users. Let $S_{m}$ be the total allocated power for the $m^{\text {th }}$ D2D user, $E_{m}$ be the extra power for the $m^{\text {th }}$ D2D user when $S_{m}$ exceeds the device's maximum power $\left(P_{m, \max }\right)$ and $R_{m}$ be the remaining power for the $m^{t h} \mathrm{D} 2 \mathrm{D}$ user that needs to be reallocated in the next round. In order to satisfy device's maximum power constraint, three cases are considered: 1) $\left.S_{m}=P_{m, \max }, 2\right)$ $S_{m}>P_{m, \max }$ and 3) $S_{m}<P_{m, \max }$. For the first case, after checking the interference and minimum rate requirement constraints, we can have the allocated power vector for those D2D users directly. For the second case, we first perform GWFPP with the corresponding $P_{m, \max }$ and then, check the interference and minimum rate requirement constraints to get their power allocation vectors. In this case, we also calculate all the $E_{m}$ that needs to be reallocated in the next round. Finally, for the third case, in order to perform GWFPP less number of times, we start with those D2D users where we can allocate the remaining power most while satisfying the required constraints. In each case, if any violation of the interference or minimum rate constraint happens, this algorithm creates a set of $\Omega$ for those subcarriers and the amount of the $R_{m}$ that needs to be reallocated in the next round. The process 
continues until all the reallocation being completed while satisfying all the constraints. The required steps for performing the power allocation are described in Fig. 4.5 and the detailed algorithm is well depicted in the Algorithm 2 description.

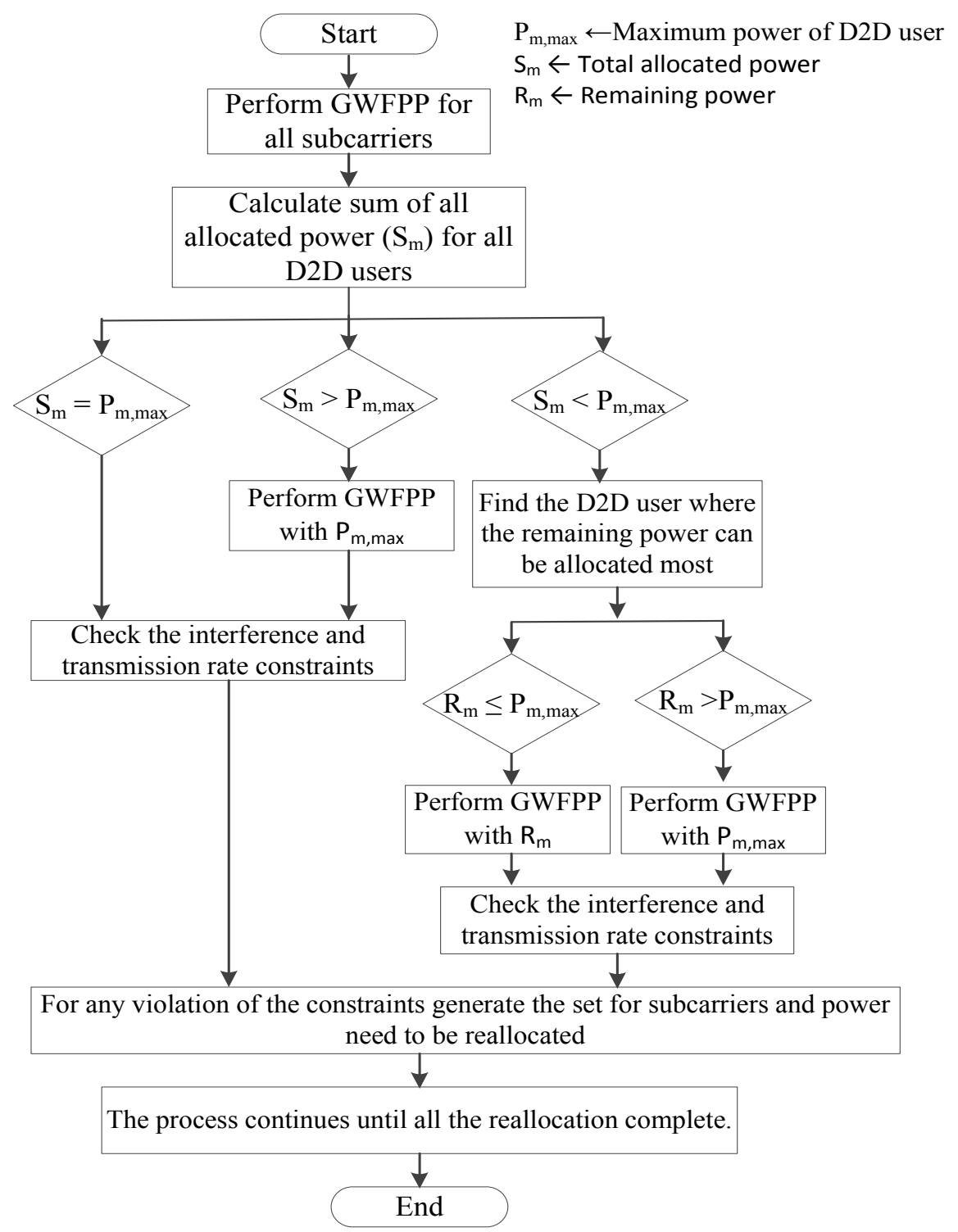

Figure 4.5: Flow chart of power allocation algorithm. 


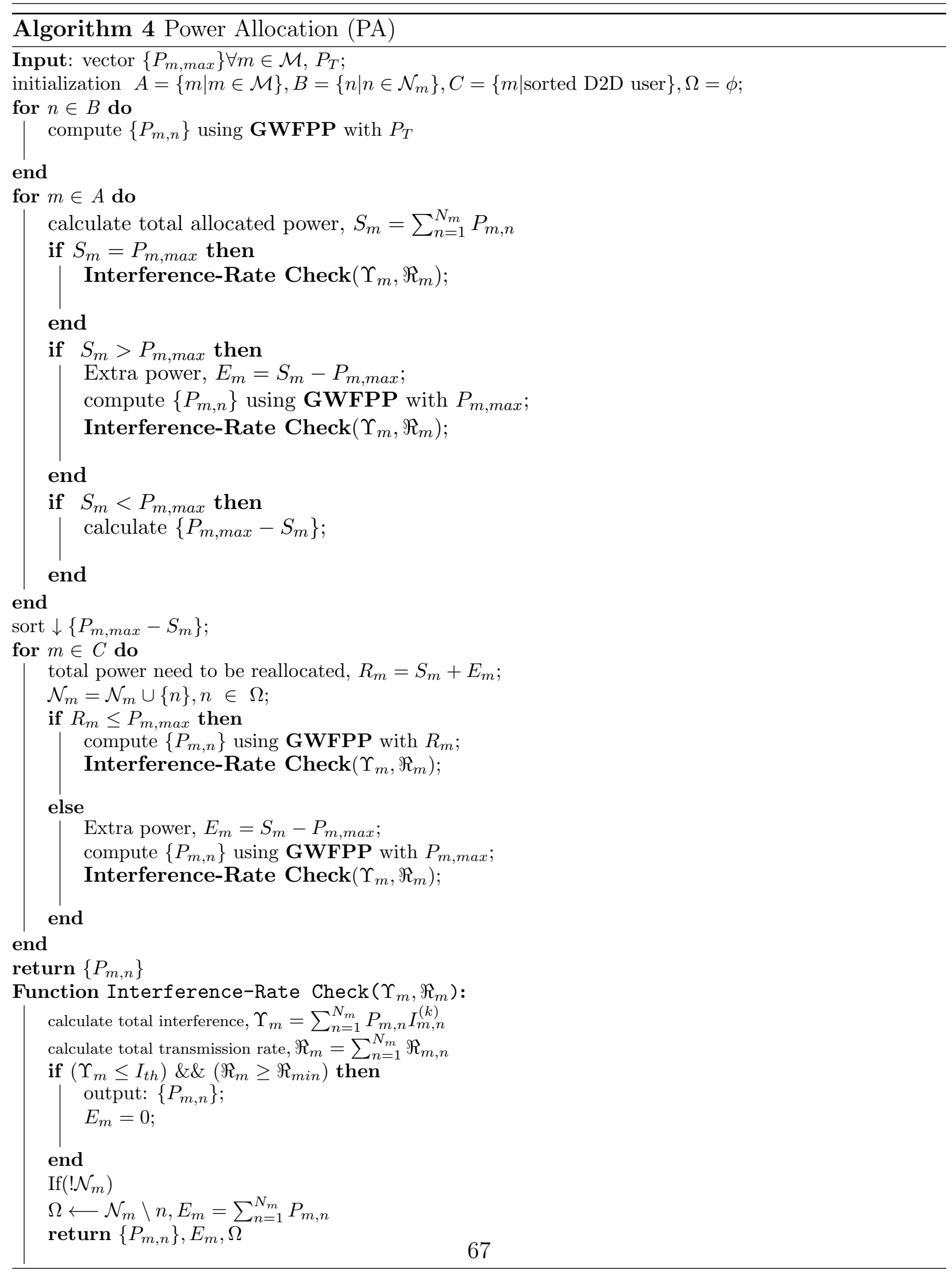




\subsection{Performance Evaluation}

In order to evaluate the performance of the subcarrier assignment and power allocation algorithm for D2D communications, we simulate a multiuser single cell system with radius $100 \mathrm{~m}$ where BS is located in the center of the cell using MATLAB. All cellular users (perform as PUs) and D2D users (perform as SUs) are uniformly distributed randomly within the cell. The distance between D2D pairs varies depending on their relative position in the cell. All other simulation parameters are considered according to Table 4.3. The simulation results are evaluated over different realization of PUs and D2D users locations, interference conditions and channel gains. Average transmitted data rates for different algorithms under consideration are obtained from several independent simulation runs.

Table 4.3: Simulation Parameters

\begin{tabular}{|c|c|}
\hline Parameters & Values \\
\hline Total no. of D2D users & $5-35$ \\
\hline Total no. of PUs & $12-24$ \\
\hline Total no. of subcarriers & $5-50$ \\
\hline Subcarrier bandwidth $(\Delta B)$ & $1.5 \mathrm{KHz}$ \\
\hline The value of $T_{s}$ & $1 \mu \mathrm{s}$ \\
\hline Path loss factor & 4 \\
\hline The value of $\delta_{d}^{2}$ & $10^{-12}$ \\
\hline Max D2D $T_{x}$ power & $0.1 \mathrm{~W}$ \\
\hline Max PU $T_{x}$ power & $0.25 \mathrm{~W}$ \\
\hline
\end{tabular}

In order to evaluate the performance of the proposed algorithm, we compared our algorithm with the most popular schemes : uniform power loading, water-filling schemes [76], and ladder/triangular profile power allocation $[117,118]$. In the uniform power loading scheme, uniform power is loaded into each subcarrier and it only consider total power constraint C2. With the total power as the power constraint $(\mathrm{C} 2)$, the power profile follows the water-filling scheme as in [76]. In ladder profile scheme [117], power is distributed in such a fashion so that the subcarriers that are adjacent to the PU bands, are given less power where as the subcarriers that are far away to the PU bands, are given more power. According to 
the triangular power allocation scheme in [118], power allocated to the subcarriers near the PUs is small and gradually increase as move towards the middle. Thus maximum power is allocated to the middle most subcarrier. Since the idea behind the schemes (ladder profile and triangular power allocation) is the same, thus we name these schemes as ladder/triangle scheme and they only considered the interference constraint C5.

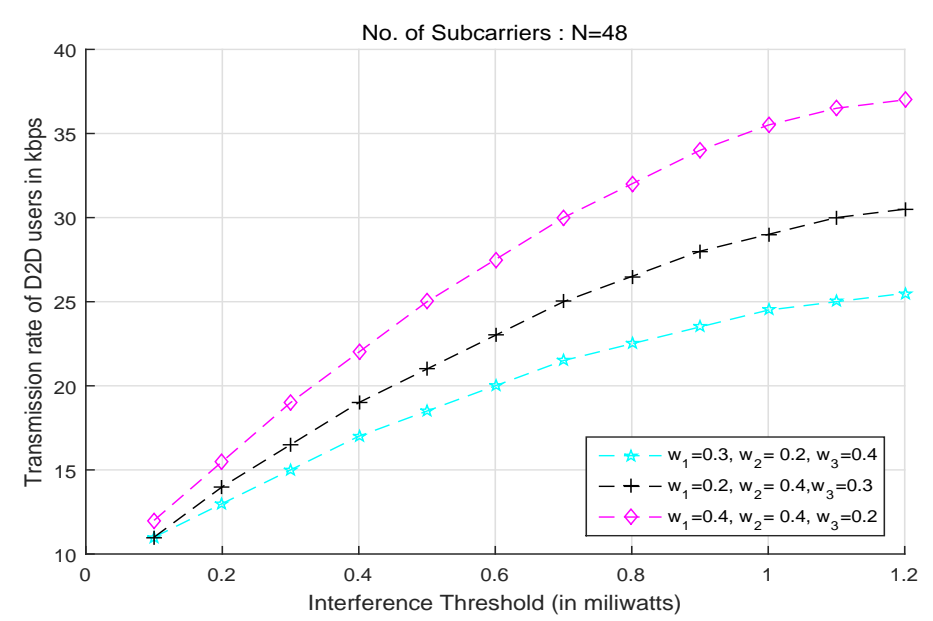

Figure 4.6: Illustration of the behavior for the proposed subcarrier assignment with different values of the weighting factors $\left(w_{1}, w_{2}, w_{3}\right)$

The behavior of the proposed subcarrier assignment strategy with different values of the weighting factors in (4.15) is illustrated in Fig. 4.6. The effect of the different weighting factors $\left(w_{1}, w_{2}, w_{3}\right)$ is revealed in the performance curves. The simulation results show that an appropriate value of the weighting factors for the threer parts in the proposed indicator can achieve superior performance. We select the values of the weighting factors $\left(w_{1}, w_{2}, w_{3}\right)$ to be $(0.4,0.4,0.2)$ in the following simulation, since this combination returns the highest sum rate of the D2D uses in the simulation range.

Fig. 4.7 shows the effect of the distance between the D2D users on subcarrier allocation where the minimum rate requirement is $50 \mathrm{kbps}$. Here, the first row represents the number of allocated subcarriers, the second row denotes the distance between the D2D users and the third row shows the transmission rate. It can be observed from Fig. 4.7 that the distance 


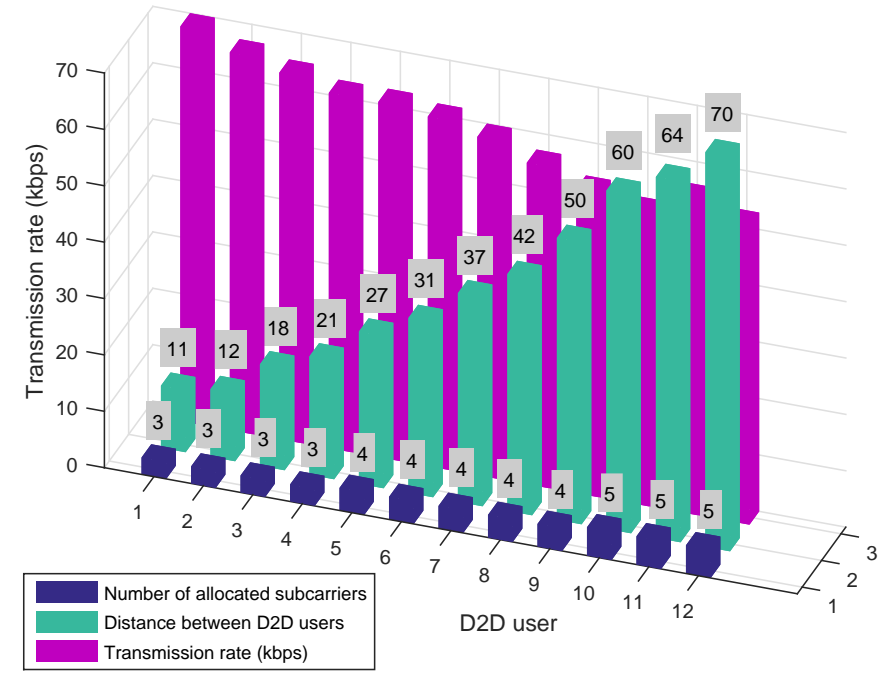

Figure 4.7: Effect of the distance between the D2D users on subcarrier allocation

between the D2D user plays an important role on the total number of subcarriers allocated to one D2D user. To satisfy the minimum rate requirements of the D2D users, closely located D2D users require less number of subcarriers compared to the distantly located D2D users. Fig. 4.7 depicts the scenario where D2D users located within 20 meters need three subcarriers to satisfy the minimum rate requirement whereas D2D users located within 70 to 80 meters range need five subcarriers to satisfy the minimum rate requirement.

In Fig. 4.8, we plotted the transmission rate of D2D users versus the interference threshold introduced to the PUs band for different schemes. As we see, the transmission rate for all the schemes increases as the interference threshold increases. Also the transmission rate achieved by the proposed scheme outperforms other three existing schemes. The reason is that the proposed scheme maintains both the power and interference constraints at all stages of the operation. The transmission rate of the uniform power loading scheme is significantly lower than the other schemes due to the violation of both of these constraints.

Fig. 4.9 shows the transmission rate achieved by the D2D users versus the number of subcarriers for the different schemes. It is obvious that as the number of subcarriers increases, the proposed scheme provides better transmission rate for D2D users than the other existing 


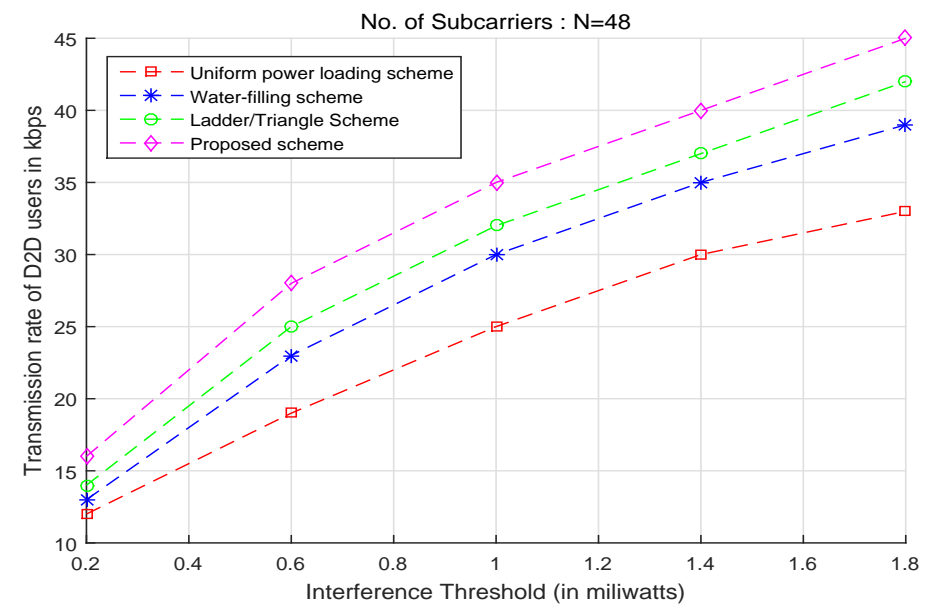

Figure 4.8: Transmission rate of D2D users versus interference threshold to the PUs for different schemes

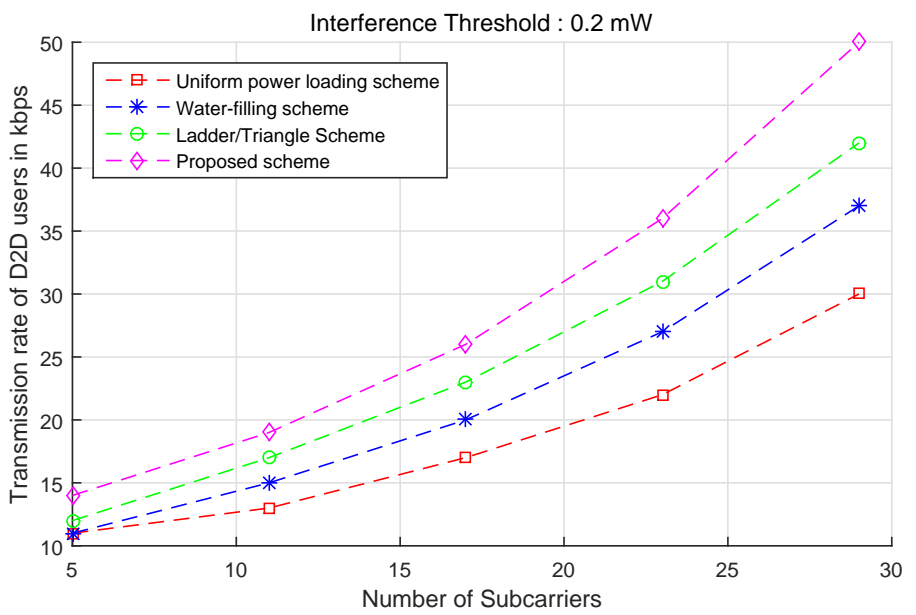

Figure 4.9: Transmission rate of D2D users versus number of subcarriers with fixed interference threshold for different schemes 
methods. The reason is that our proposed ASA scheme is adaptive in nature due to the three different parts in the used metric.

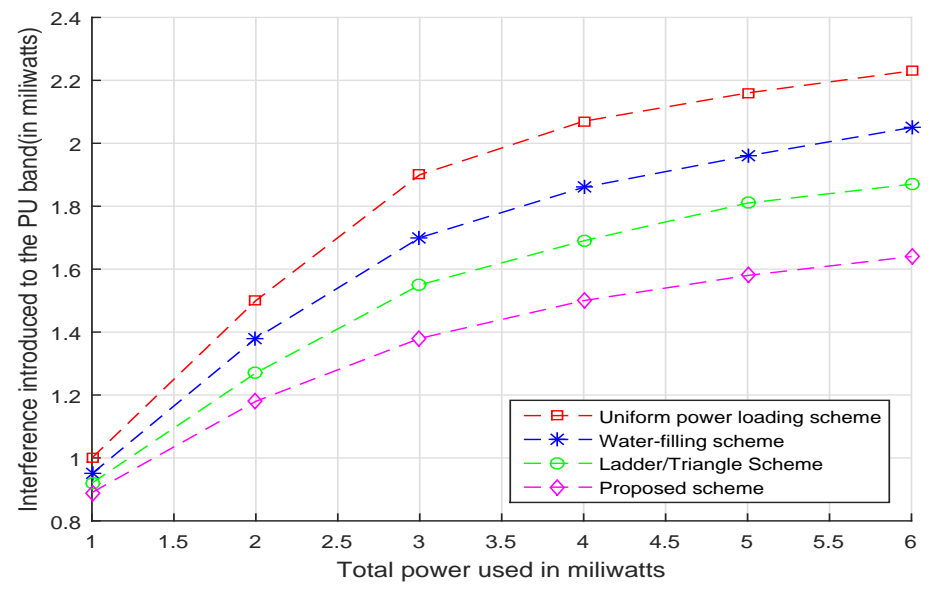

Figure 4.10: Total power budget versus interference introduced to the PU band for different schemes.

Fig. 4.10 presents the interference introduced to the PU band versus total power budget for different schemes. As the total power budget increases, the interference generated to the PU band by the uniform power loading and water-filling schemes are severe due to not taking the interference constraints into account. On the other hand, our proposed scheme is able to load power into multi-D2D users providing increased transmission rate while always keeping the interference introduced to the PU band below a specified threshold.

Fig. 4.11 illustrates the scenario of the total transmission rate versus the number of D2D users for the different schemes. It can be observed that as we increase the number of D2D users, the total transmission rate of our proposed scheme outperforms the other existing schemes while maintaining different constraints. When the number of D2D users remain small, the total transmission rate graph for all the schemes follow the linear pattern with a larger slope. However, for large number of D2D users, the total transmission rate graph for all the schemes become little flat with a smaller slope. This is obvious that as we increase the number of D2D users, the interference constraint become dominant in this region and 


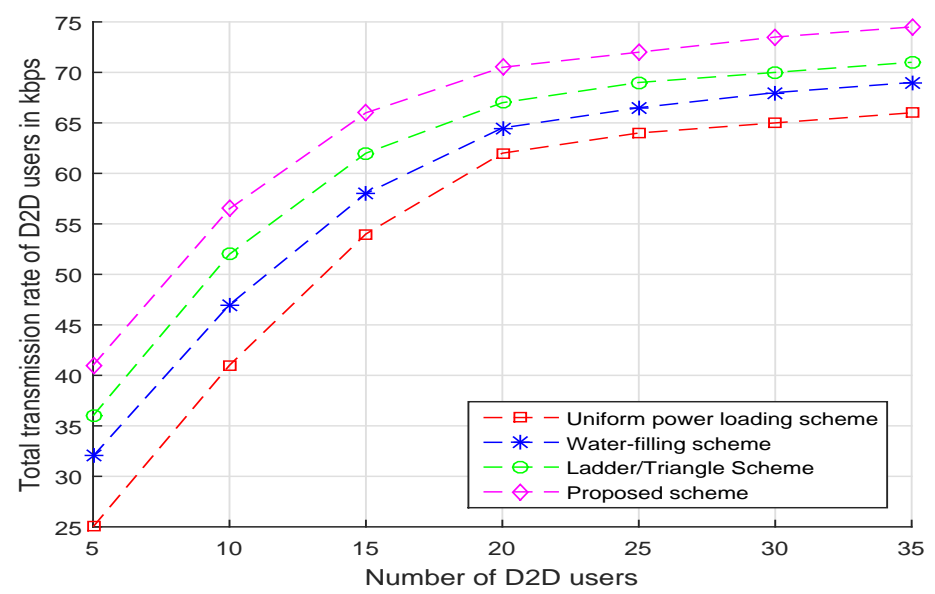

Figure 4.11: Total transmission rate versus number of D2D users for the different schemes

the total transmission rate does not increase as the number of D2D users increase. However, in both region (linear or little flat), our proposed scheme provides superior performance than the other schemes.

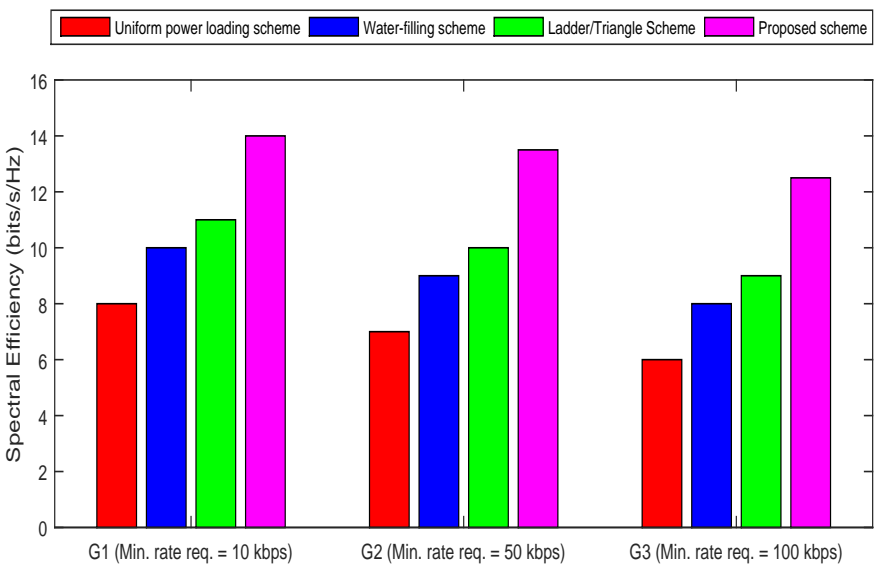

Figure 4.12: Performance of different schemes for different minimum rate requirements

Fig. 4.12 investigates the performance of different algorithms for different minimum rate requirements. Here, three different groups (e. g. G1, G2 and G3) are categorized for three different minimum rate requirement values (e. g. $10 \mathrm{kbps}, 50 \mathrm{kbps}$ and $100 \mathrm{kbps}$ ) to evaluate the algorithms performance in terms of spectral efficiency. Spectral efficiency is defined as the 
total transmission rate divided by the bandwidth of the spectrum dedicated to the D2D user with unit bits/s/Hz. The comparison result presents that due to an increase in the minimum rate requirement, for the same constraints, the spectral efficiency decreases. This is because less number of D2D users are admitted due to high rate requirement threshold and stringent power and interference constraints. However, still the proposed scheme outperforms the other schemes. From Fig. 4.12, the improvement (in \%) of the proposed scheme on spectral efficiency compared to the other schemes for different minimum rate requirements has been calculated which is well depicted in Table 4.4 .

Table 4.4: Improvement (in \%) of the proposed scheme on spectral efficiency compared to other schemes.

\begin{tabular}{|c|c|c|}
\hline Groups & Other schemes & Improvement \\
\hline \multirow{2}{*}{ G1 (Min. rate req. 10kbps) } & Uniform power loading & $43 \%$ \\
\cline { 2 - 3 } & Water-filling & $29 \%$ \\
\cline { 2 - 3 } & Ladder/Triangle & $22 \%$ \\
\hline \multirow{2}{*}{ G2 (Min. rate req. 50kbps) } & Uniform power loading & $48 \%$ \\
\cline { 2 - 3 } & Water-filling & $33 \%$ \\
\cline { 2 - 3 } & Ladder/Triangle & $26 \%$ \\
\hline \multirow{2}{*}{ G3 (Min. rate req. 100kbps) } & Uniform power loading & $52 \%$ \\
\cline { 2 - 3 } & Water-filling & $36 \%$ \\
\cline { 2 - 3 } & Ladder/Triangle & $28 \%$ \\
\hline
\end{tabular}

\subsection{Chapter Summary}

This Chapter provides the system model to jointly employ CR technology and D2D communication in cellular networks and construct the RA problem considering five constraints. Describes a two-stage approach where a new ASA scheme is designed first and then, a novel PA scheme is developed utilizing geometric water-filling (GWF) approach. Present simulation results that validate the proposed solutions than the existing schemes. 
Chapter 5

Energy Efficient Power Allocation in

Underlay and Overlay Cognitive

Device-to-Device Communications 
CHAPTER 5. ENERGY EFFICIENT POWER ALLOCATION IN UNDERLAY AND OVERLAY COGNITIVE DEVICE-TO-DEVICE COMMUNICATIONS

\section{$5.1 \quad$ Introduction}

There have been several research efforts found in the literature [119-121] on the energy efficient RA schemes for CRNs. The paper [119] presents a subchannel and power allocation protocol that maximizes the energy efficiency (EE) of transmissions from a cognitive base station operating in the TV white spaces. The proposed two-step solution to the EE maximization problem satisfies users minimum rate requirements and keeps the interference to the PUs in the neighboring areas below a specified threshold. The energy-efficient RA is studied in [120] in the orthogonal frequency division multiplexing (OFDM)-based CR networks under channel uncertainty including fairness issue. In [121], the authors proposed two energy efficient RA schemes that utilize the sensing information.

On the other hand, due to the benefit of D2D communication, International Mobile Telecommunications (IMT)- advanced standard systems, such as long term evolution (LTE) and WiMax, allow D2D communication sharing the same radio resources with the cellular network to increase the spectral efficiency [19]. D2D communication is, in fact integrated into LTE-Advanced networks in [100].

RRA for D2D communication underlaying cellular networks are currently extensively investigated by researchers in terms of energy efficiency [122-127]. The paper [122] demonstrates how the integration of D2D communication in cellular systems operating under dynamic time division duplex (TDD) can improve energy efficiency. In this paper, the authors perform joint optimization of mode selection, uplink/downlink transmission period, and power allocation to minimize the transmission energy consumption while satisfying the traffic requirement. In [123], the authors have investigated the energy-efficient power control for D2D communications with underlaying cellular networks, where both the total EE and individual EE have been considered. Another energy-efficient RA problem is studied in [124] for D2D communication with underlaying cellular networks, which aims to maximize the minimum weighted energy efficiency of D2D links while guaranteeing minimum data rates for cellular links. On the other hand, joint RA and power control scheme has been derived 
CHAPTER 5. ENERGY EFFICIENT POWER ALLOCATION IN UNDERLAY AND OVERLAY COGNITIVE DEVICE-TO-DEVICE COMMUNICATIONS

in [125] to maximize the EE of D2D communications. In [126], the average EE and spectral efficiency of multihop D2D communications have been analyzed under orthogonal sharing mode. Joint channel and power allocation scheme for D2D communication has been studied in [127] in order to optimize the expected data during battery lifetime of user equipments (UEs), instead of the traditional EE.

From the literature survey, it is found that, most recent works focus on exploiting D2D communication in cellular networks under different constraints. Only a few efforts $[19,110-$ 113] have been incorporated D2D communication with CR technology to jointly maximize the spectrum efficiency, not to mention the EE. Very few efforts $[128,129]$ have been observed in the literature addressing the EE. The paper [128] has developed energy-efficien RA algorithms for both underlay and overlay communications, in the presence of co-existing multiple-input single-output (MISO) primary link with a multiple-input multiple-output (MIMO) secondary link. Authors of this paper have solved the RA problem by maximizing the SUs EE subject to a minimum rate requirement of the PU and tackled it by fractional programming. In [129], the energy optimization problem for cognitive D2D communications has been investigated considering mutual preferences and satisfactions of users and proposed an EE stable matching algorithm based on game and matching theories.

\subsection{System Model}

Let us consider the downlink of a single cell cognitive D2D system where $K$ cellular users (PUs) coexists with $M$ D2D users (SUs) as shown in Fig. 5.1. It is assumed that $K$ active PUs occupy a total of $N$ orthogonal channels and there is no co-channel interference among the PUs. Usually, the number of PUs is much higher than the number of D2D users. Hence we assume $K>>M$ and a PU can share an orthogonal channel with only one D2D user. In the following, the index sets of active PUs, D2D users and orthogonal channels are denoted as $\mathcal{K}=\{1, \ldots, k, \ldots, K\}, \mathcal{M}=\{1, \ldots, m, \ldots, M\}$ and $\mathcal{N}=\{1, \ldots, n, \ldots, N\}$ respectively. In 
CHAPTER 5. ENERGY EFFICIENT POWER ALLOCATION IN UNDERLAY AND OVERLAY COGNITIVE DEVICE-TO-DEVICE COMMUNICATIONS

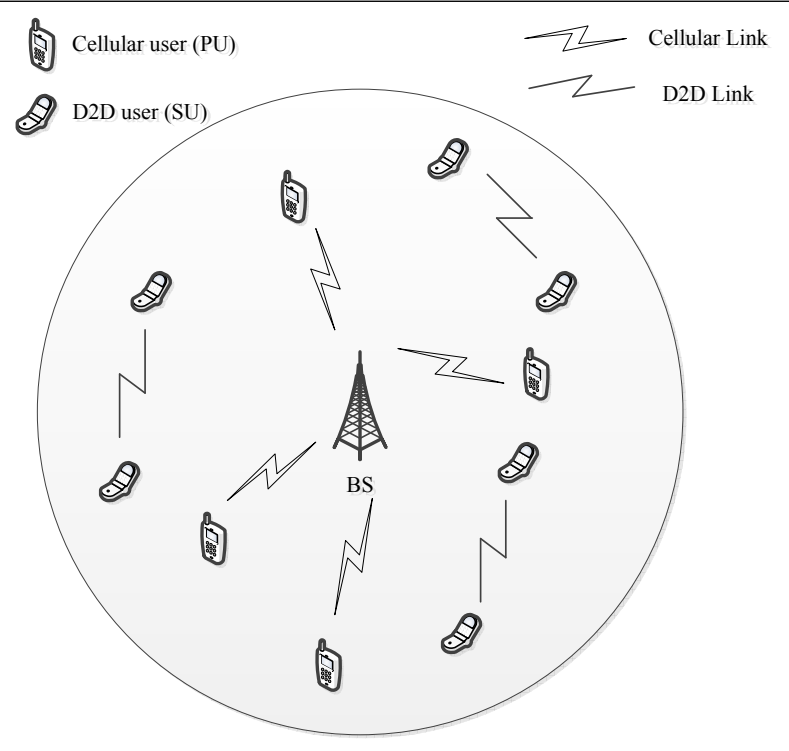

Figure 5.1: A D2D enabled cognitive radio system.

addition, it is assumed that both PUs and D2D pairs meet their minimum QoS requirements in terms of SINR, and the base station (BS) has the perfect CSI information of all the links.

Besides the distance based pathloss model, both the fast fading due to multi-path propagation and slow fading due to shadowing are also considered in this channel model. Thus, the channel gain between the $k^{\text {th }} \mathrm{PU}$ transmitter and the $m^{\text {th }} \mathrm{D} 2 \mathrm{D}$ user receiver on the $n^{\text {th }}$ channel can be expressed as [129]

$$
g_{k, m}^{n}=\eta \beta_{k, m}^{n} \zeta_{k, m}^{n} \mathcal{D}_{k, m}^{-\alpha},
$$

where, $\eta$ is the pathloss constant determined by system parameters, $\beta_{k, m}^{n}$ is fast fading gain with an exponential distribution, $\zeta_{k, m}^{n}$ is the slow fading gain with a log-normal distribution, $\alpha$ is the pathloss exponent, and $\mathcal{D}_{k, m}$ is the transmission distance between the $k^{\text {th }} \mathrm{PU}$ and the $m^{\text {th }}$ D2D user receiver. Similarly, we can denote the channel gain between the $m^{\text {th }}$ D2D user transmitter and the $k^{\text {th }} \mathrm{PU}$ receiver on the $n^{\text {th }}$ channel as $g_{m, k}^{n}$. Also, the channel gain of the $k^{\text {th }} \mathrm{PU}$ transmitter and receiver on the $n^{\text {th }}$ channel as $g_{k k}^{n}$ and the $m^{\text {th }}$ D2D user transmitter and receiver on the $n^{\text {th }}$ channel as $g_{m m}^{n}$. 
CHAPTER 5. ENERGY EFFICIENT POWER ALLOCATION IN UNDERLAY AND OVERLAY COGNITIVE DEVICE-TO-DEVICE COMMUNICATIONS

In this work, two approaches are considered to manage the coexistence among the primary and secondary system: the underlay and the overlay approach. In the underlay approach, a secondary system may reuse the primary resource as long as the primary rate remains above the threshold $T_{1}$ set by the primary system. The underlay protocol popular in cognitive radio systems [130-132], has observed application in D2D systems that operate in the reuse mode recently [133]. In this underlay D2D mode, a pair of neighbouring devices can autonomously set up a D2D connection reusing resource presently used by a cellular user (PU), granted that the cellular link is still able to preserve its required communication rate.

On the other hand, the overlay approach regards a different situation. An underlying assumption in the underlay approach is that the transmission rate of the primary link (without the presence of the secondary link) is larger than the required communication rate $T_{1}$. Therefore, the secondary system may reuse the primary system resources as long as its interference does not affect the primary transmission rate to drop below $T_{1}$. However, if the primary system faces poor propagation conditions, or if it wanted to preserve energy transmitting at a lower power, it might occur that even without any interference from the secondary system, the transmission rate of the primary link might be already below $T_{1}$. A solution for this problem is that the primary system may get the assistance of a secondary (D2D) transmitter, that is probably located in a more suitable position with better channel condition. In these situations, the secondary (D2D) system becomes a relay for the primary message, and in exchange can reuse the primary resource to transmit its own message [46].

Therefore, the underlay and overlay approaches can be exploited in complementary fashion. If the primary system enjoys good condition and able to have much higher than the required communication rate, then the underlay approach should be utilized, because it is simpler, need no coordination between the primary and D2D systems. If instead, the transmission rate of the primary link is below the required communication rate, then the overlay approach can be applied to raise the primary system transmission rate. In many practical scenarios, the resulting rate increase is large enough to allow the primary system to guarantee 
CHAPTER 5. ENERGY EFFICIENT POWER ALLOCATION IN UNDERLAY AND OVERLAY COGNITIVE DEVICE-TO-DEVICE COMMUNICATIONS

the required communication rate $[128,134-136]$.

In this paper, we consider the following power consumption model. It captures not only the transmission power of the D2D users but also the power consumption along the entire circuitry including mixer, analog-to-digital converter (ADC), digital-to-analog converter (DAC), filters, and digital signal processing (DSP) blocks. The circuit power consumption can be divided into the static background power consumption $P_{\text {back }}$ and the dynamic power consumption $\varepsilon R$ that is dependent on the communication transmission rate $R$ and the power consumption per unit rate $\varepsilon$. Therefore, the circuit power consumption can be expressed as,

$$
p_{\text {cir }}=\varepsilon R+P_{\text {back }} .
$$

Now, the total power consumption by the D2D users can be written as,

$$
p_{\mathrm{t}}=\sum_{m \in \mathcal{M}, n \in \mathcal{N}} \frac{1}{\theta} P_{m, n}+2 p_{\text {cir }}
$$

where $p_{\mathrm{t}}$ is composed of the transmission power over all of the channels, and the circuit power of both the $\mathrm{D} 2 \mathrm{D}$ transmitter and receiver, i.e., $2 p_{\text {cir }} . \theta$ is the power amplifier efficiency, i.e., $0<\theta<1$.

\subsubsection{Underlay Approach}

In the underlay mode D2D users share the resources with the PUs. This is possible since D2D communication links are usually short-range so that interference to PUs is rather low. In addition, extensive researches [137] have shown that the inter-cell interference can be kept very low by power control and proper radio resource management. Thus in this work, we confine our focus to the intra-cell interference between the PU and the D2D users only. By fully utilizing the knowledge of the CSI, power allocation can be executed to attain efficient spectral usage and power saving in cellular networks.

We suppose that $x_{1}$ and $x_{2}$ be the transmitted signal from the PU and the D2D users 
CHAPTER 5. ENERGY EFFICIENT POWER ALLOCATION IN UNDERLAY AND OVERLAY COGNITIVE DEVICE-TO-DEVICE COMMUNICATIONS

respectively. Then the received signals at the PU and D2D user receivers at a given time instant can be written as,

$$
\begin{aligned}
& y_{1}=\sqrt{P_{k, n}} g_{k k}^{n} x_{1}+\sqrt{P_{m, n}} g_{m, k}^{n} x_{2}+\sigma_{1} \\
& y_{2}=\sqrt{P_{m, n}} g_{m m}^{n} x_{2}+\sqrt{P_{k, n}} g_{k, m}^{n} x_{1}+\sigma_{2}
\end{aligned}
$$

respectively. Here, $P_{k, n}$ and $P_{m, n}$ indicate the transmission power of the $k^{\text {th }} \mathrm{PU}$ and the $m^{\text {th }}$ D2D user on the $n^{\text {th }}$ channel respectively. Furthermore, $\sigma_{1} \sim \mathcal{C N}\left(0, \sigma_{p}^{2}\right)$ and $\sigma_{2} \sim \mathcal{C N}\left(0, \sigma_{d}^{2}\right)$ model the additive white Gaussian noise (AWGN) for the PU and the D2D user receiver, respectively.

The channel gain to noise ratio of the $k^{\text {th }} \mathrm{PU}$ and the $m^{\text {th }} \mathrm{D} 2 \mathrm{D}$ user on the $n^{\text {th }}$ channel can be expressed as,

$$
\begin{aligned}
\Gamma_{k, n} & =\frac{\left|g_{k k}^{n}\right|^{2}}{P_{m, n}\left|g_{m, k}^{n}\right|^{2}+\sigma_{1}} \\
\Gamma_{m, n} & =\frac{\left|g_{m m}^{n}\right|^{2}}{P_{k, n}\left|g_{k, m}^{n}\right|^{2}+\sigma_{2}}
\end{aligned}
$$

respectively.

Now, the achievable rate in $\mathrm{b} / \mathrm{s} / \mathrm{Hz}$ (i.e., normalized with respect to the channel bandwidth) of the $k^{\text {th }} \mathrm{PU}$ and the $m^{\text {th }} \mathrm{D} 2 \mathrm{D}$ user on the $n^{\text {th }}$ channel can be written as

$$
\begin{gathered}
R_{k, n}=\log _{2}\left(1+\Gamma_{k, n} P_{k, n}\right) \\
R_{m, n}=\log _{2}\left(1+\Gamma_{m, n} P_{m, n}\right)
\end{gathered}
$$

respectively. 


\subsubsection{Overlay Approach}

In the overlay approach, the D2D transmitter assists the PU by relaying the primary message and, in exchange enjoys the privilege to use the primary spectrum. Any of the relaying techniques can be employed in the D2D system. Here, half-duplex amplify-and-forward (AF) relaying technique is considered. Unlike decode-and-forward (DF), the use of AF does not require any decoding operation at the D2D transmitter, which results in a lower complexity, lower-delay, and lower-feedback requirement, and a simpler design [138]. The considered digital implementation of AF has been explicitly proposed for the LTE-A standard [139]. Moreover, time division duplex mode is utilized to split the incoming signal from the forwarded signal. In the first phase, the PU employs maximum ratio transmission to transmit its signal $x_{1}$, as in the underlay approach. During this phase, the signal $x_{1}$ is also received by the D2D transmitter, which, in the second phase precodes and then transmits this amplified signal together with its own message. The superposition of these two signals is labeled $z_{2}$.

Now, the received signals at the PU receiver and at the D2D user transmitter in the first phase are expressed as,

$$
\begin{gathered}
y_{1}^{(1)}=\sqrt{P_{k, n}} g_{k k}^{n} x_{1}+\sigma_{1}^{(1)} \\
y_{d_{t}}=\sqrt{P_{m, n}} g_{k, m_{t}}^{n} x_{1}+\sigma_{d_{t}}
\end{gathered}
$$

where, $g_{k, m_{t}}^{n}$ is the channel gain the PU and the D2D user transmitter on the $n^{\text {th }}$ channels, $\sigma_{1}^{(1)} \sim \mathcal{C N}\left(0, \sigma_{p}^{2}\right)$ and $\sigma_{d_{t}} \sim \mathcal{C N}\left(0, \sigma_{d_{t}}^{2}\right)$ model the AWGN at the PU receiver and D2D user transmitter, respectively.

Similarly, the received signals at the PU and the D2D receivers in the second phase are expressed as,

$$
\begin{aligned}
& y_{1}^{(2)}=\sqrt{P_{m, n}} g_{m, k}^{n} z_{2}+\sigma_{1}^{(2)} \\
& y_{2}^{(2)}=\sqrt{P_{m, n}} g_{m m}^{n} z_{2}+\sigma_{2}^{(2)}
\end{aligned}
$$


CHAPTER 5. ENERGY EFFICIENT POWER ALLOCATION IN UNDERLAY AND OVERLAY COGNITIVE DEVICE-TO-DEVICE COMMUNICATIONS

where, $\sigma_{1}^{(2)} \sim \mathcal{C N}\left(0, \sigma_{p}^{2}\right)$ and $\sigma_{2}^{(2)} \sim \mathcal{C N}\left(0, \sigma_{d}^{2}\right)$ model the AWGN at the PU and the D2D user receiver, respectively, and $z_{2}$ is given by $z_{2}=\gamma y_{d_{t}}+x_{2}$, where $\gamma=\sqrt{\frac{P_{m, n}}{g_{k, m_{t}}^{n} P_{k, n}+\sigma_{d_{t}}}}$ is the amplifying factor at the AF relaying system.

Now, the signal received at the $\mathrm{PU}$ receiver in the two phases can be written as

$$
\begin{aligned}
y_{1}= & \sqrt{P_{k, n}}\left\{g_{k k}^{n}+\sqrt{P_{m, n}} g_{m, k}^{n} \gamma g_{k, m_{d_{t}}}^{n}\right\} x_{1}+\sqrt{P_{m, n}} g_{m, k}^{n} x_{2}+ \\
& \sqrt{P_{m, n}} g_{m, k} \gamma \sigma_{d_{t}}+\sigma_{1}^{(1)}+\sigma_{1}^{(2)}
\end{aligned}
$$

and the signal received at the $\mathrm{D} 2 \mathrm{D}$ receiver can be written as,

$$
\begin{aligned}
y_{2}= & \sqrt{P_{m, n}} g_{m m} \sqrt{P_{k, n}} \gamma g_{k, m_{t}}^{n} x_{1}+\sqrt{P_{m, n}} g_{m m}^{n} x_{2}+ \\
& \sqrt{P_{m, n}} g_{m m} \gamma \sigma_{d_{t}}+\sigma_{2}^{(2)} .
\end{aligned}
$$

The channel gain to noise ratio of the $k^{\text {th }} \mathrm{PU}$ and the $m^{\text {th }} \mathrm{D} 2 \mathrm{D}$ user on the $n^{\text {th }}$ channel can be expressed as

$$
\begin{aligned}
\Gamma_{k, n} & =\frac{\left|g_{k k}^{n}\right|^{2}+P_{m, n}\left|g_{m, k}^{n}\right|^{2} \gamma\left|g_{k, m_{d_{t}}}^{n}\right|^{2}}{P_{m, n}\left|g_{m, k}^{n}\right|^{2}+P_{m, n}\left|g_{m, k}^{n}\right|^{2} \gamma \sigma_{d_{t}}+\sigma_{1}^{(1)}+\sigma_{1}^{(2)}} \\
\Gamma_{m, n} & =\frac{\left|g_{m m}^{n}\right|^{2}}{\left|g_{m m}^{n}\right|^{2} P_{k, n} \gamma\left|g_{k, m_{t}}^{n}\right|^{2}+\left|g_{m m}^{n}\right|^{2} \gamma \sigma_{d_{t}}+\sigma_{2}^{(2)}}
\end{aligned}
$$

respectively.

Now, the achievable rate in b/s/Hz (i.e., normalized with respect to the channel bandwidth) of the $k^{\text {th }} \mathrm{PU}$ and the $m^{\text {th }}$ D2D user on the $n^{\text {th }}$ channel can be written as

$$
\begin{aligned}
R_{k, n} & =\frac{1}{2} \log _{2}\left(1+\Gamma_{k, n} P_{k, n}\right) \\
R_{m, n} & =\frac{1}{2} \log _{2}\left(1+\Gamma_{m, n} P_{m, n}\right)
\end{aligned}
$$

respectively, where the factor " $\frac{1}{2}$ " indicates that the transmission takes only half of the protocol time. 
CHAPTER 5. ENERGY EFFICIENT POWER ALLOCATION IN UNDERLAY AND OVERLAY COGNITIVE DEVICE-TO-DEVICE COMMUNICATIONS

\subsection{Power Allocation problem and solution}

In this section, we will first formulate the power allocation problem, and then describe the corresponding power allocation algorithms.

\subsubsection{Problem Formulation}

For both the underlay and overlay approaches, the EE of the D2D users can be defined as follows:

$$
E E_{d}=\frac{\sum_{m \in \mathcal{M}, n \in \mathcal{N}} R_{m, n}}{p_{\mathrm{t}}}
$$

Then, the EE optimization problem can be formulated as follows:

$$
\begin{aligned}
& \max _{P_{m, n} \geq 0} E E_{d}\left(P_{m, n}\right) \\
& \text { subject to: } \\
& C 1: \sum_{k \in \mathcal{K}, n \in \mathcal{N}} R_{k, n} \geq T_{1} \\
& C 2: \sum_{m \in \mathcal{M}, n \in \mathcal{N}} R_{m, n} \geq T_{2} \\
& C 3: \quad P_{m, n} \leq P_{\text {max }}^{d} \quad \forall m \in \mathcal{M}, \forall n \in \mathcal{N}
\end{aligned}
$$

where, constraint $\mathrm{C} 1$ and $\mathrm{C} 2$ ensure that the $\mathrm{PU}$ and $\mathrm{D} 2 \mathrm{D}$ users' rates remain above the thresholds $T_{1}$ and $T_{2}$, and constraint $\mathrm{C} 3$ is the D2D users' power constraint, with $P_{\max }^{d}$ the D2D user's maximum feasible power.

\subsubsection{Solution Approaches}

The EE optimization problem in (5.21) is a non-linear fractional optimization problem where, the objective functions have the numerators as non-linear functions and the denominators in a sum power form. Therefore, the fractional objective function in (5.21), which is a nonconvex 
CHAPTER 5. ENERGY EFFICIENT POWER ALLOCATION IN UNDERLAY AND OVERLAY COGNITIVE DEVICE-TO-DEVICE COMMUNICATIONS

function, turns the problem even more complicated and computationally intractable. Due to the fractional form of the EE optimization problem, the Karush-Kuhn-Tucker (KKT) conditions can not directly be utilized to solve it. Hence, existing algorithms seldom directly solve the EE maximization problem, including the widely-utilized Dinkelbach algorithm [140].

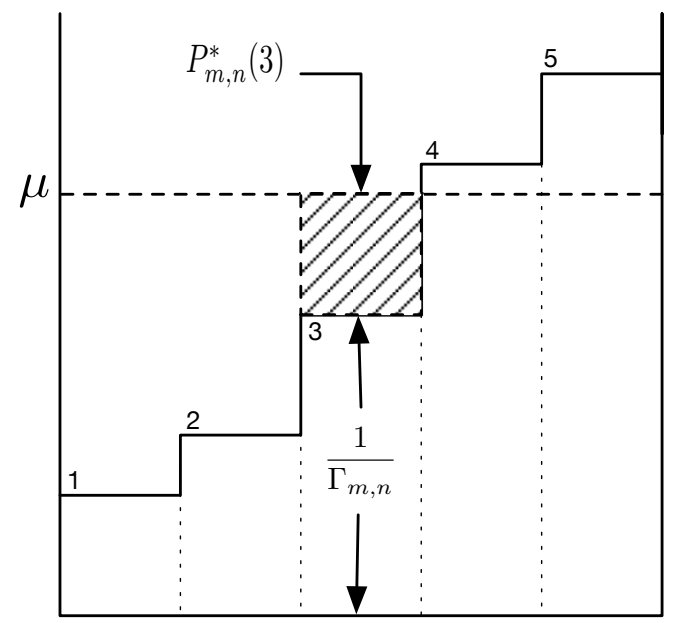

(a)

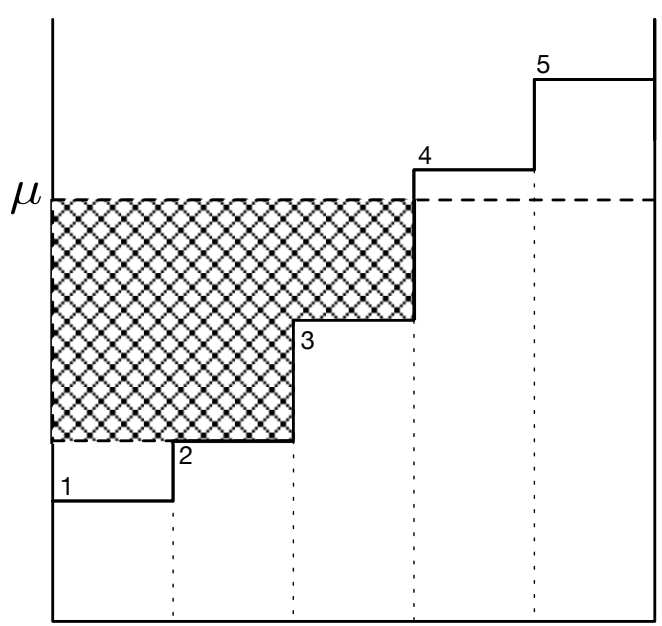

(b)

Figure 5.2: Illustration for the GWF algorithm (a) Illustration of water level step $j^{*}=3$, allocated power for the third step $P_{m, n}^{*}(3)$, and step depth is $\frac{1}{\Gamma_{m, n}}$ and (b) Illustration of $P(j)$ (shadowed area, representing the total power above step $j$ ) when $j=2$.

In this section, a proven power allocation algorithm based on GWF approach [141] has been utilized to solve the EE optimization problem. In order to illustrate GWF technique, a water tank with equal width and monotonically increasing $N$ steps/stairs is considered that correspond to the $N$ channels. Fig. 2 gives an illustration of the GWF approach. Let $\lambda_{m, n}$ denotes the "step depth" of the $m^{\text {th }}$ D2D user of the $n^{\text {th }}$ stair/channel which is the height of the $n^{\text {th }}$ step to the bottom of the tank, and is given as:

$$
\lambda_{m, n}=\frac{1}{\Gamma_{m, n}}, \forall m \in M, \forall n \in N .
$$

Since the sequence $\Gamma_{m, n}$ is sorted as monotonically decreasing, the step depth of the stairs 
CHAPTER 5. ENERGY EFFICIENT POWER ALLOCATION IN UNDERLAY AND OVERLAY COGNITIVE DEVICE-TO-DEVICE COMMUNICATIONS

indexed as $\{1, \ldots, N\}$ is monotonically increasing. When water (power) $P_{T}$ is poured into the tank, a water level $\mu$ is obtained. The optimal power allocation to each channel corresponds to the area above the stair up to the water level.

The main idea of the GWF algorithm [78] is summarized as follows. Let $j^{*}$ denote the index of the highest (in terms of shadow) step under water, then it can be written as:

$$
j^{*}=\max \{j \mid P(j)>0,1 \leq j \leq N\}
$$

where, $P(j)$ is the whole water volume above the $j^{\text {th }}$ step. Considering the geometrical relationship, $P(j)$ can be written as,

$$
P(j)=\left\{P_{T}-\sum_{n=1}^{j-1}\left(\frac{1}{\Gamma_{m, j}}-\frac{1}{\Gamma_{m, n}}\right)\right\}^{+}, \forall m \in \mathcal{M}, \forall j \in \mathcal{N} .
$$

Then, the allocated power for the $m^{\text {th }} \mathrm{D} 2 \mathrm{D}$ user to the $j^{*}$ step is

$$
P_{m, j^{*}}=\frac{1}{j^{*}} P\left(j^{*}\right),
$$

and the complete solution is given by

$$
P_{m, n}= \begin{cases}P_{m, j^{*}}+\left(\frac{1}{\Gamma_{m, j^{*}}}-\frac{1}{\Gamma_{m, n}}\right) & 1 \leq n \leq j^{*} \\ 0, & j^{*}<n \leq N .\end{cases}
$$

The GWF algorithm is denoted by $\operatorname{GWF}\left(\left\{\Gamma_{m, n}\right\}, P_{T}\right)$, i.e., the mapping from $\left\{\left\{\Gamma_{m, n}\right\}, P_{T}\right\}$ to $\left\{j^{*}, P\left(j^{*}\right)\right\}$.

On the other hand, the P-GWF algorithm [142] has been proposed to compute the sum power minimization problem with the transmission rate requirement constraint. The basic sum power problem can be stated as: given the target transmission rate $T>0$, a group of 
CHAPTER 5. ENERGY EFFICIENT POWER ALLOCATION IN UNDERLAY AND OVERLAY COGNITIVE DEVICE-TO-DEVICE COMMUNICATIONS

powers $\left\{P_{m, n}\right\}$ need to be found to satisfy,

$$
\begin{aligned}
\min \sum_{m \in \mathcal{M}, n \in \mathcal{N}} P_{m, n} \\
\text { subject to: } C 1: 0 \leq P_{m, n}, \forall m, \forall n ; \\
C 2: \sum_{m \in \mathcal{M}, n \in \mathcal{N}} R_{m, n} \geq T .
\end{aligned}
$$

In [142], algorithm P-GWF has been proven to provide the optimal solution to the problem (5.27). Algorithm P-GWF provides the mapping of $\left(\left\{\Gamma_{m, n}, T\right\}\right)$ to the exact solution $\left\{P_{m, n}\right\}$ and $j^{*}$.

Here, we develop an algorithm denoted by EE-GWF to solve the optimization problem (4.8), based on the GWF approach and P-GWF method. In the first step, GWF algorithm has been used to find the highest water (power) level index that denotes the maximum number of the channels for power allocation. In the second step, P-GWF algorithm has been utilized in two rounds in order to find a group of power levels that has been satisfied by both the minimum rate requirement for the PU and D2D users i. e., $T_{1}$ and $T_{2}$.

Now, the final step is to find the EE-optimal power allocation for the target problem in (4.8) that satisfies the water-filling like architecture with the water tank model. Thus, a water tank with unit width and monotonically increasing steps is utilized to illustrate the geometric relationship of the variables as in Fig. 5.3. Let, again $N$ denote the total number of the steps that correspond to the $N$ channels in the tank. Assume a certain amount of water is poured into the tank, making water level $\mu$ between the $L^{\text {th }}$ and the $(L+1)^{\text {th }}$ step. For the $n^{\text {th }}$ step $(n \leq L)$, the power allocated is $P_{m, n}=\mu-\lambda_{m, n}$. An auxiliary variable $\Delta P$, shown as the shadowed area in Fig. 5.3, denotes the entire volume of the water (power) above the $L^{\text {th }}$ step. Since each step is assumed to have a unit width, the allocated power for the $L^{\text {th }}$ step is,

$$
P_{L}=\frac{\Delta P}{L} .
$$




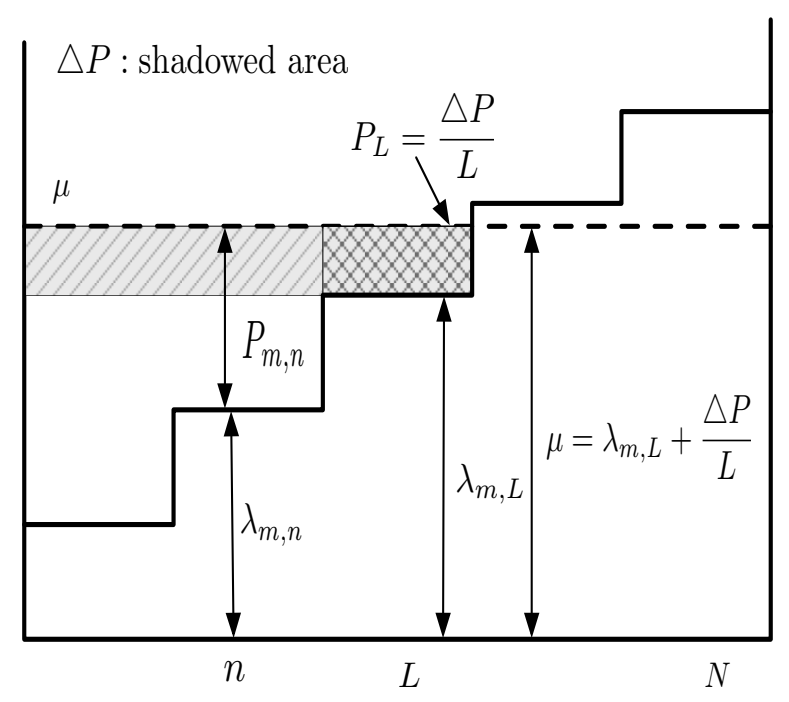

Figure 5.3: Illustration of geometric relation and auxiliary variable $\Delta P$.

From the geometric relationship, $\Delta P$ lies between $\left[\Delta P_{\min }, \Delta P_{\max }\right]$, where $\Delta P_{\min }=0, \Delta P_{\max }=$ $L\left(\lambda_{m, L+1}-\lambda_{m, L}\right) . \Delta P_{\min }$ occurs when the water level $\mu$ is at the $L^{\text {th }}$ step, and $\Delta P_{\max }$ happens when the water level $\mu$ reaches the $(L+1)^{\text {th }}$ step.

Then, for a given $L$, let us introduce a function $F(\Delta P)$, where $\Delta P$ is the variable with the geometric meaning shown as in Fig. 5.3,

$$
\begin{aligned}
F(\Delta P)= & \left(\Delta P+\lambda_{m, L} \cdot L\right) \log \left(\lambda_{m, L}+\frac{\Delta P}{L}\right)-\left(\lambda_{m, L}+\frac{\Delta P}{L}\right) \\
& \sum_{j=1}^{L} \log \left(\lambda_{m, j}\right)-P_{T}, \forall m \in \mathcal{M}
\end{aligned}
$$

where $P_{m, n}$ is the allocated power for the $m^{\text {th }}$ D2D user on the $n^{\text {th }}$ channel,

$$
P_{m, n}=\left(\lambda_{m, L}-\lambda_{m, n}\right)+\frac{\Delta P}{L}, \forall 1 \leq n \leq L, \forall m \in \mathcal{M}
$$

In [141], it is proven that $F(\Delta P)$ exhibits the desired monotonically increasing property 
CHAPTER 5. ENERGY EFFICIENT POWER ALLOCATION IN UNDERLAY AND OVERLAY COGNITIVE DEVICE-TO-DEVICE COMMUNICATIONS

in the range of $\left(\Delta P_{\min }, \Delta P_{\max }\right)$. Hence, the EE-optimal power allocation is equivalent to,

$$
\min |F(\Delta P)|
$$

subject to: $0 \leq \Delta P \leq L\left(\lambda_{m, L+1}-\lambda_{m, L}\right)$.

The optimal solution to (5.31) is classified into three cases which are illustrated in Fig. 5.4.

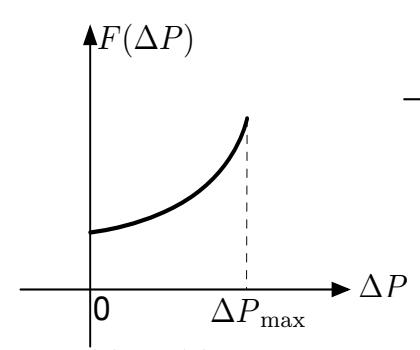

(1) $: F(0)>0$

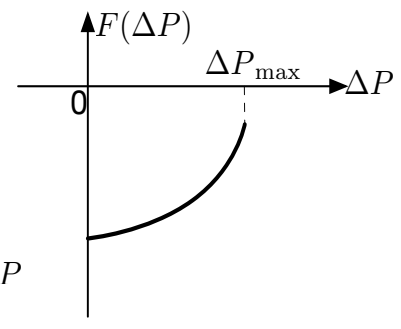

$(2): F\left(\Delta P_{\max }\right)<0$

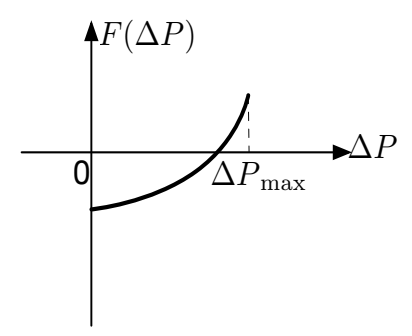

$(3): F(0) \cdot F\left(\Delta P_{\max }\right)<0$

Figure 5.4: Illustration for solving conditions (5.31).

When the optimal solution to (5.31) is not at the boundaries i. e., $F(0)<0$ and $F\left(\Delta P_{\max }\right)>0$, then $\Delta P$ can be calculated through the following iteration,

$$
\Delta P_{i+1}=\Delta P_{i}-\frac{F\left(\Delta P_{i}\right)}{F^{\prime}\left(\Delta P_{i}\right)}, \forall i \in \mathcal{I}^{+},
$$

where, $\mathcal{I}^{+}$is the set of non-negative integers. The subscript of $\Delta P_{i}$ is the iteration index. Details of the iteration steps can be found in [141].

Next, the required steps for performing the power allocation in EE-GWF algorithm are described in Fig. 5.5 and the detailed algorithm is well depicted in the Algorithm description.

\section{Algorithm EE-GWF:}




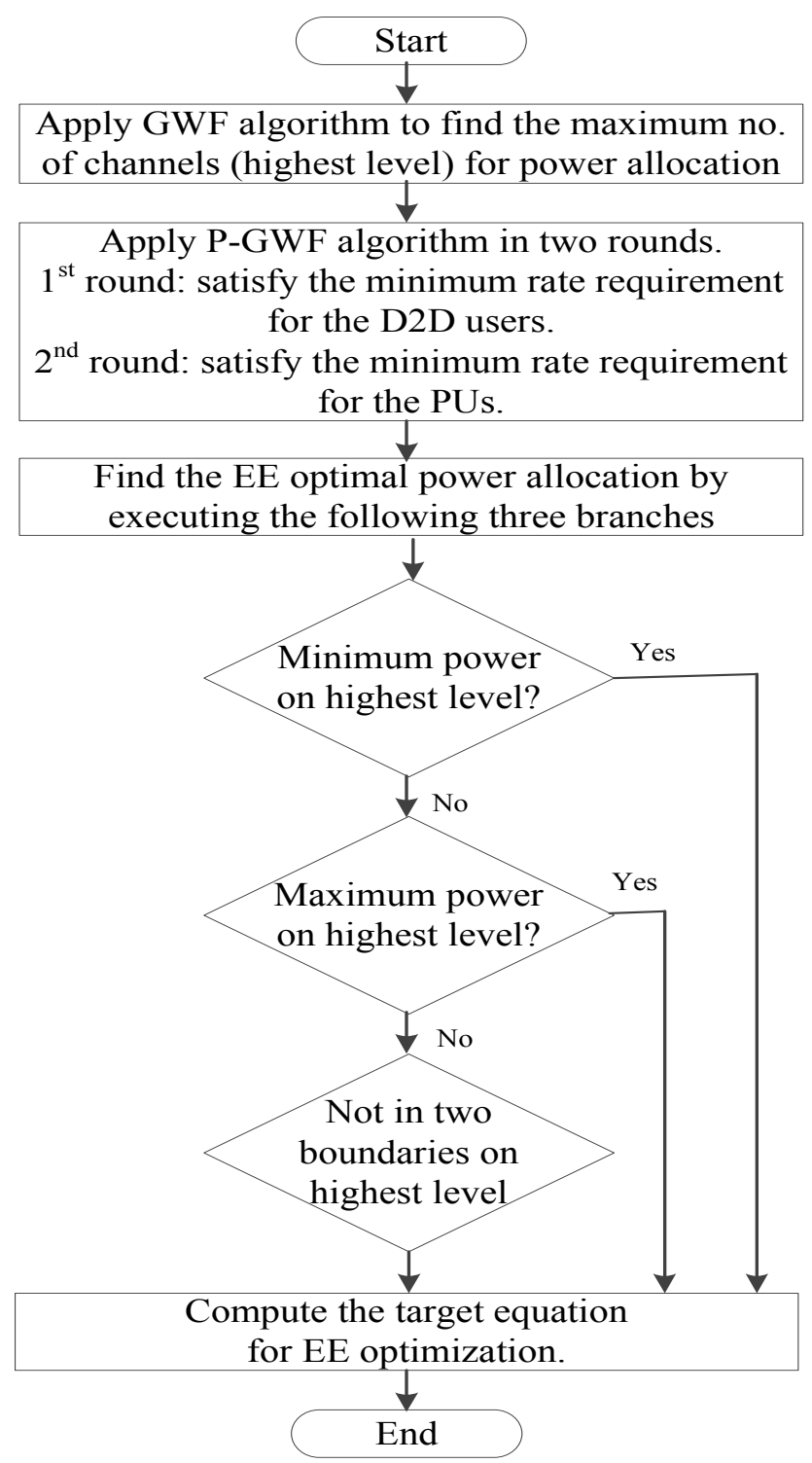

Figure 5.5: Flow chart of EE-GWF algorithm. 
1) Pre-processing:

$$
\begin{aligned}
& \left\{j^{*}, P\left(j^{*}\right)\right\}=\operatorname{GWF}\left(\left\{\Gamma_{m, n}\right\}, P_{T}\right), \\
& j^{*} \rightarrow W \text { and } \frac{P(W)}{W}+\frac{1}{\Gamma_{W}} \rightarrow \frac{1}{\Gamma_{W+1}}, \\
& \text { 1st round : }\left\{j^{*},\left\{P_{m, n}\right\}\right\}=\operatorname{P-GWF}\left(\left\{\Gamma_{m, n}\right\}, T_{2}\right), \\
& \text { 2nd round : }\left\{j^{*},\left\{P_{k, n}\right\}\right\}=\operatorname{P-GWF}\left(\left\{\Gamma_{k, n}\right\}, T_{1}\right), \\
& \min \left\{j \mid P_{m, j^{*}}+\frac{1}{\Gamma_{m, j^{*}}}<\frac{1}{\Gamma_{m, j}}, 1 \leq j \leq N\right\}-1 \rightarrow \underline{j} . \\
& P_{m, j^{*}}+\frac{1}{\Gamma_{m, j^{*}}} \rightarrow \lambda_{m, j}, \text { for } 1 \leq j \leq j ; \\
& \text { while } \frac{1}{\Gamma_{m, j}} \rightarrow \lambda_{m, j}, \text { for } \underline{j}<j \leq N .
\end{aligned}
$$

where the symbol " $\rightarrow$ " denotes the assignment operation and a new index $\underline{j}$ is utilized after applying P-GWF to guarantee both the minimum rate requirements constraints.

1) Input: Let $i=\underline{j}$ and assigning a

$N+1-\underline{j} \times N$ matrix $\mathbf{P}$ a null matrix.

2) Loop for $i$ from 1 to $W$ : for each loop, one of the following three branches being executed to update the $i^{\text {th }}$ row of the matrix $\mathbf{P}$ :

From now on we are omiting $m$ in the subscript of $P$ in order to reduce notation complexity.

2.1) If $F\left(\triangle P_{\min }\right)=F(0)>0$,

$$
P_{i, x}=\frac{1}{\Gamma_{i}}-\frac{1}{\Gamma_{x}}, \quad x=1, \cdots, i,
$$

and then go to Step 3); else

$2.2)$ if $F\left(\triangle P_{\max }\right)=F\left(i\left(\frac{1}{\Gamma_{i+1}}-\frac{1}{\Gamma_{i}}\right)\right)<0$,

$$
P_{i, x}=\frac{1}{\Gamma_{i+1}}-\frac{1}{\Gamma_{x}}, \quad x=1, \cdots, i,
$$


CHAPTER 5. ENERGY EFFICIENT POWER ALLOCATION IN UNDERLAY AND OVERLAY COGNITIVE DEVICE-TO-DEVICE COMMUNICATIONS

and then go to item 3); else

2.3) if

$$
F(0) \cdot F\left(i\left(\frac{1}{\Gamma_{i+1}}-\frac{1}{\Gamma_{i}}\right)\right)<0,
$$

then solving $\triangle P$ by using equation (5.32) and

$$
P_{i, x}=\frac{\triangle P}{i}+\left(\frac{1}{\Gamma_{i}}-\frac{1}{\Gamma_{x}}\right), \quad j=1, \cdots, i .
$$

3) If $i=W$, compute

$$
i^{*}=\arg \max _{\{i \mid 1 \leq i \leq W\}}\left\{\frac{\frac{1}{2} \sum_{n=1}^{N} \log _{2}\left(1+\Gamma_{n} P_{i, n}\right)}{2 p_{\mathrm{cir}}+\sum_{n=1}^{N} \frac{1}{\theta} P_{i, n}}\right\},
$$

and then output $\bar{P}_{j}=P_{i^{*}, j}, \forall j$; else let $i+1 \rightarrow i$ and go to Step 2).

\subsection{Performance Evaluation}

\subsubsection{Simulation Setup}

In order to evaluate the performance of the developed power allocation algorithm, a multiuser single cell system is simulated with radius $500 \mathrm{~m}$. The BS is located in the center of the cell. All cellular users (PUs) and D2D users (SUs) are randomly distributed with uniform distribution within the cell. The distances between D2D pairs vary depending on their relative position in the cell. The cellular link transmits with power at $20 \mathrm{dBm}$, and requires a minimum rate $T_{1}$ set as a percentage $\mathrm{T}$ of the $\mathrm{PU}$ transmission rate without the presence of the D2D system. All other simulation parameters are considered according to Table I. The simulation results are evaluated over different realization of the PUs and D2D user locations, rate constraints and channel gains. Average EE optimization for both the underlay and overlay approaches are obtained from several independent simulation runs.

Let us first analyze the performance of the developed algorithm for the underlay approach. 
CHAPTER 5. ENERGY EFFICIENT POWER ALLOCATION IN UNDERLAY AND OVERLAY COGNITIVE DEVICE-TO-DEVICE COMMUNICATIONS

Table 5.1: Simulation Parameters

\begin{tabular}{|c|c|}
\hline Parameters & Values \\
\hline Total no. of D2D users & $5-35$ \\
\hline Total no. of PUs & $10-50$ \\
\hline Max D2D $T_{x}$ power & $20 \mathrm{dBm}$ \\
\hline Max PU $T_{x}$ power & $10 \mathrm{dBm}, 20 \mathrm{dBm}$ \\
\hline Max D2D transmission distance & $10-90 \mathrm{~m}$ \\
\hline Power consumption per unit rate $\varepsilon$ & $1 \mathrm{~mW} / \mathrm{kbps}$ \\
\hline Static background power consumption $P_{\text {back }}$ & $5 \mathrm{dBm}$ \\
\hline Power amplifier efficiency $\theta$ & $30 \%$ \\
\hline Pathloss exponent $\alpha$ & 4 \\
\hline Pathloss constant $\eta$ & $10^{-2}$ \\
\hline $\begin{array}{c}\text { Shadowing } \zeta_{k, m}^{n} \text { (standard deviation } \\
\text { of a log-normal distribution) }\end{array}$ & $8 \mathrm{~dB}$ \\
\hline $\begin{array}{c}\text { Multi-path fading } \beta_{k, m}^{n}(\text { the mean of } \\
\text { an exponential distribution) }\end{array}$ & 1 \\
\hline Noise power $\sigma$ & $-144 \mathrm{dBm}$ \\
\hline
\end{tabular}

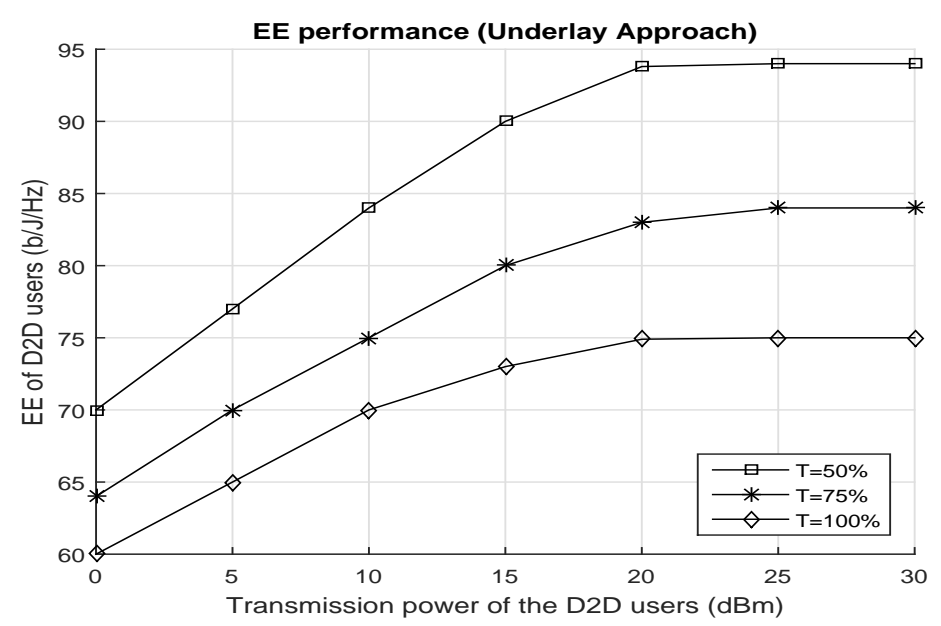

Figure 5.6: Energy Efficiency versus transmission power of the D2D users with underlay approach

In Fig. 5.6, the EE of the developed scheme versus the transmission power of the D2D users is illustrated under different minimum rate requirements (e. g. $T=50 \%, 75 \%$ and $100 \%$ ). As the transmission power of the D2D users increases the EE of the developed scheme also increases almost linearly first and then it becomes saturated. Saturation happens because 
CHAPTER 5. ENERGY EFFICIENT POWER ALLOCATION IN UNDERLAY AND OVERLAY COGNITIVE DEVICE-TO-DEVICE COMMUNICATIONS

it exceeds the maximum transmission power limit of the D2D users. Also, as the minimum rate requirement increases, the EE performance decreases. The reason behind that is less number of D2D users are admitted due to high minimum rate requirement.

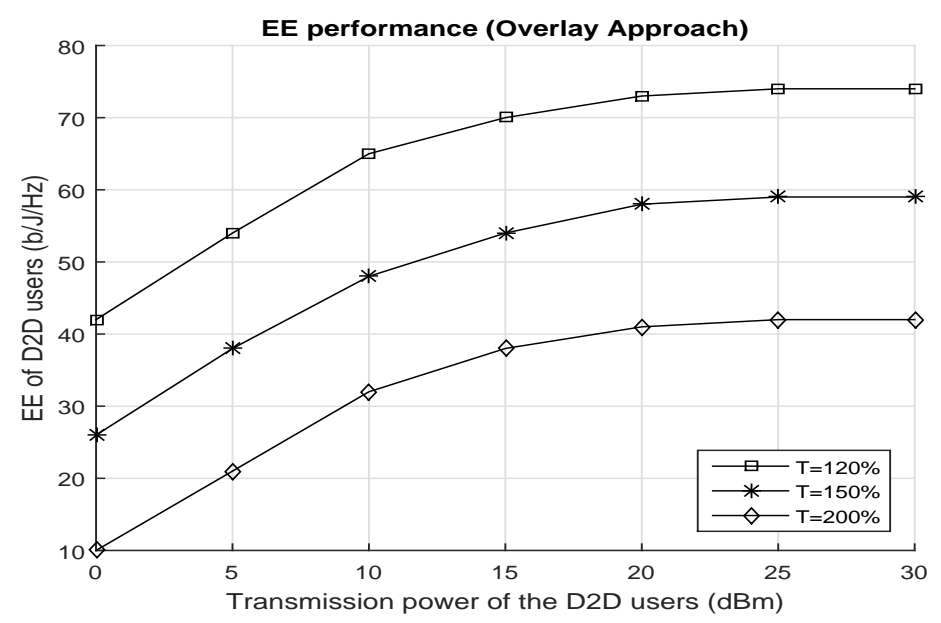

Figure 5.7: Energy Efficiency versus transmission power of the D2D users under overlay approach

Next, let us focus on the overlay approach where the PUs need the help of the D2D transmitter to meet its own minimum rate requirement. Similar simulation parameters have been considered for analysing the overlay performance as in the underlay approach. All the users' positions are randomly generated as earlier. However, the D2D transmitter has been placed closer to the PU receiver than the PU transmitter. Thus, the distance between the D2D transmitter and the PU receiver is kept between $15 \%$ and $75 \%$ of the distance between the PU transmitter and the PU receiver. All other parameters remain the same except that the PU transmission power is kept at $10 \mathrm{dBm}$. In Fig. 5.7, the EE of the developed scheme versus the transmission power of the D2D users is illustrated under different minimum rate requirements (e. g. $T=120 \%, 150 \%$ and 200\%). Similar behaviour holds for the Fig. 5.7 as in underlay case.

Fig. 5.8 depicts the affects of the distance between the D2D pairs on the EE performance of the developed solution. It can be observed that the EE performance decreases in both 


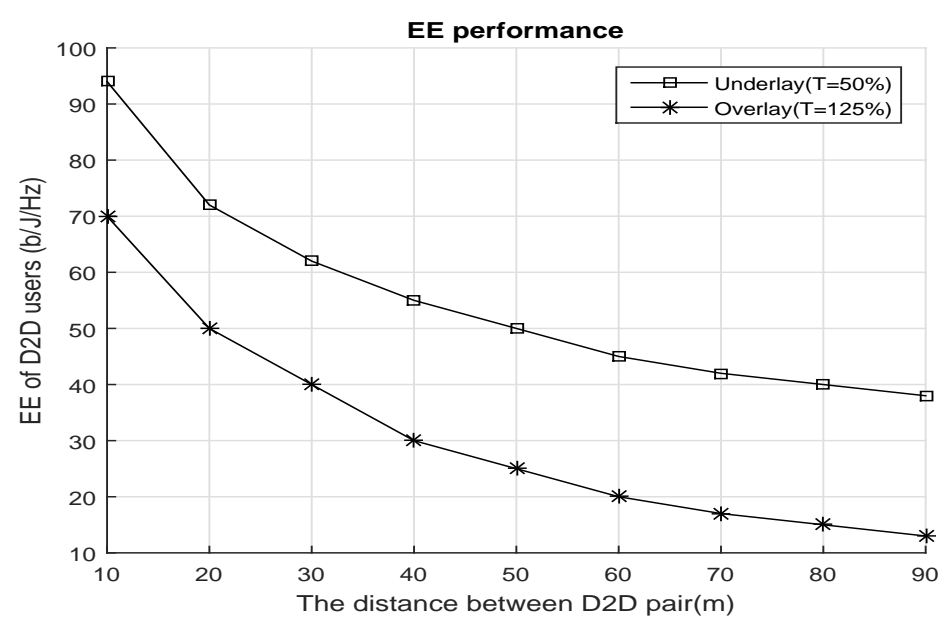

Figure 5.8: Energy Efficiency versus the distance between the D2D pair

the underlay and overlay schemes with the increase of the distance between the D2D pairs. This is because the total fading (path-loss, shadowing and small-scale fading) increases with the distance between the D2D pairs. The effect of the distance on the energy efficiency in overlay case is more than in the underlay case due to the requirement regarding the distance between the D2D transmitter and the PU receiver.

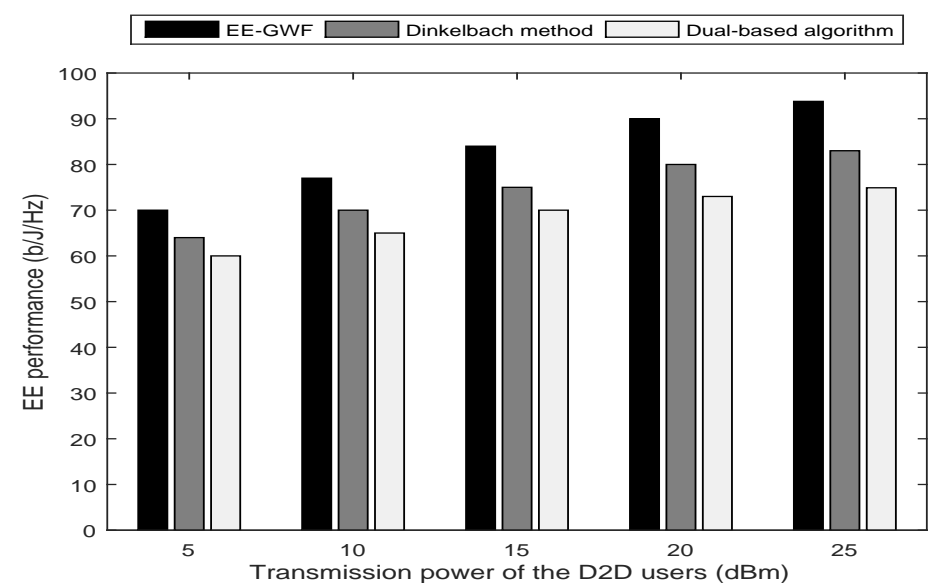

Figure 5.9: EE performance versus the transmission power of the D2D users

Next, in order to evaluate the performance of the developed solution, the EE-GWF algorithm is compared with the widely-adopted Dinkelbach method [125] and the dual- 
CHAPTER 5. ENERGY EFFICIENT POWER ALLOCATION IN UNDERLAY AND OVERLAY COGNITIVE DEVICE-TO-DEVICE COMMUNICATIONS

based method [124] from the existing literature. For EE-GWF algorithm, only the underlay approach is considered since it is well utilized in the literature. Fig. 5.9 presents the EE performance versus the transmission power of the D2D users under different algorithms. It is observed from Fig. 5.9 that as the transmission power of the D2D users increases the EE performance increases for all three algorithms. However, the EE-GWF algorithms outperforms the other two algorithms.

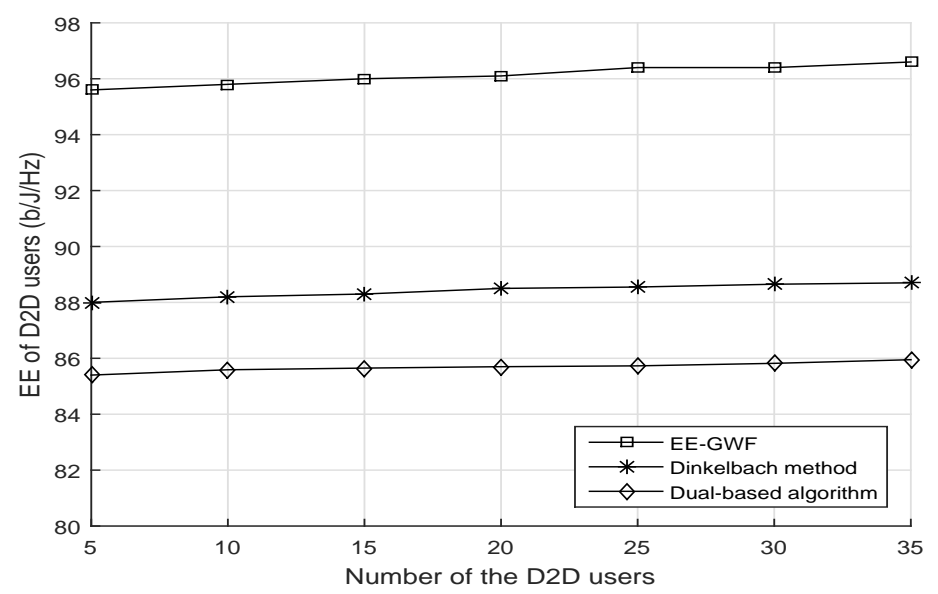

Figure 5.10: EE performance versus the number of the D2D users

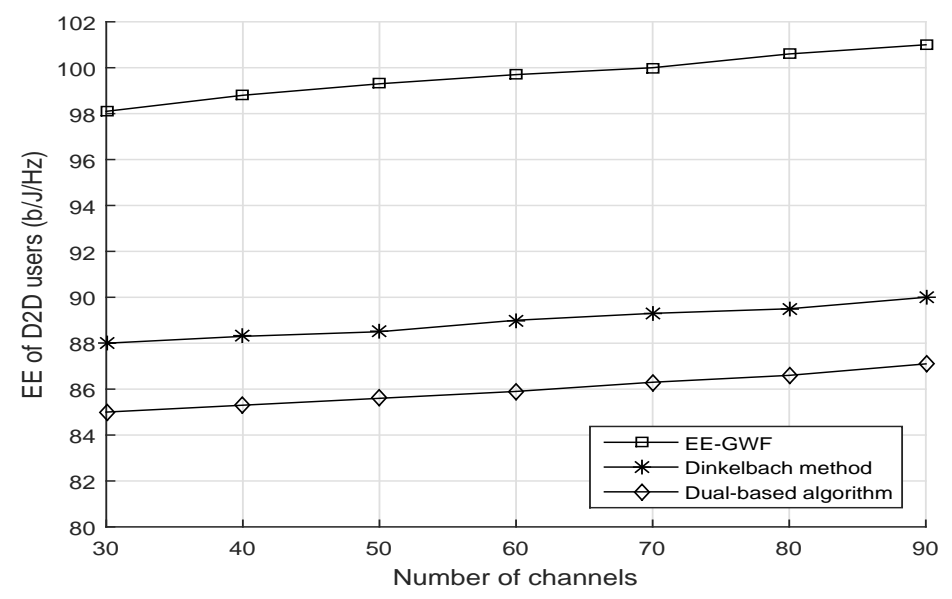

Figure 5.11: EE performance of the D2D users versus the number of the channels

Fig. 5.10 and Fig. 5.11 describe the two scenarios of the EE performance of the D2D users 
CHAPTER 5. ENERGY EFFICIENT POWER ALLOCATION IN UNDERLAY AND OVERLAY COGNITIVE DEVICE-TO-DEVICE COMMUNICATIONS

versus the number of the D2D users and the number of the channels for different algorithms, respectively. In both figures, it is observed that as the number of D2D users or the number of channels increases, the EE performance of the D2D users increases. In Fig. 5.10, the increase in the EE performance of the D2D users is very little as compared to the EE performance in Fig. 5.11. This is because as the number of D2D users increases in Fig. 5.10, they are all utilizing the same fixed channels. On the other hand, in Fig. 5.11, the increase in the EE performance is more because more resources (channels) are available for the fixed number of the D2D users. In Fig. 5.10 and Fig. 5.11, it is also observed that the developed algorithm EE-GWF outperforms the other two schemes. The reason is that in the EE-GWF algorithm only the channels with high channel gains are considered for the power allocation.

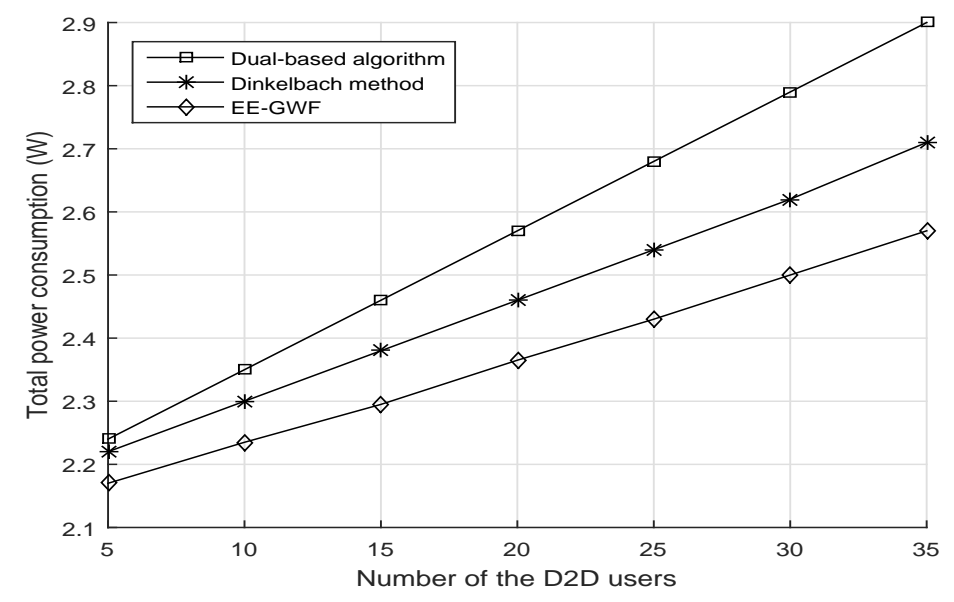

Figure 5.12: Total power consumption versus the number of the D2D users

Fig. 5.12 shows the total power comsumption by differents algorithms versus the number of D2D users for certain EE target. It is observed from Fig. 5.12 that the total power comsumption increases with the increase of the number of the D2D users and the EE-GWF algorithm consumes least amount of power than the other two algorithms. This is because the algorithm EE-GWF allocate more power to the wireless channels that have less fading. Thus, the EE-GWF allows better utilization of the available resources and saves power. 
CHAPTER 5. ENERGY EFFICIENT POWER ALLOCATION IN UNDERLAY AND OVERLAY COGNITIVE DEVICE-TO-DEVICE COMMUNICATIONS

\subsection{Chapter Summary}

This chapter investigates the energy-efficient power allocation problem of co-existing D2D and cellular users considering both underlay and overlay CR approaches. In both the (underlay and overlay) spectrum sharing schemes, the optimization problem is formulated as the EE maximization of the $\mathrm{D} 2 \mathrm{D}$ users subject to the minimum rate requirement for both the D2D users and the cellular users. A proven power allocation algorithm based on the GWF approach is utilized to solve this EE optimization problem. Contrast to the widely used Dinkelbach method for EE maximization, this algorithm can directly compute the optimal solution by applying the geometry-based approach, with exactness and low-degree polynomial complexity. Simulation results reveal the benefits of the proposed algorithm. 


\section{Chapter 6}

\section{Conclusion and Future Work}




\subsection{Conclusion}

Spectrum crunch escalates as wireless communication solutions, both human and machine centric, being deployed overwhelmingly and require more and more bandwidth. In the realm of such severe spectrum scarcity a sustainable solution for the spectrum crunch is essential. The CRN solution, that enables intelligent spectrum sharing and dynamic spectrum access could provide a serious long term solution. This is further facilitated by the dominance of software control in wireless systems, both at the transceiver level and the network level.

This thesis discusses certain key issues upon which a practical CRN may be built. The texonomy for the RA in CRNs is well researched in this thesis. Finally, the co-existance of the D2D communication in CRNs is investigated in terms of spectral efficiency and energy efficiency.

In detail, Chapter 1 presents an overview of cognitive radio networks and defines overlay, underlay and interweave type CRNs. The basic elements for the operation of CR systems and the main functions of those elements to support intelligent and efficient utilization of frequency spectrum are also envisioned here. This chapter also discusses about the D2D communication as a promising paradigm for the next generarion wireless networks.

Chapter 2 provides a taxonomy for the centralized and distributed RA process in CRNs for both infrastructure based or ad-hoc type CRNs. The resource allocation is a multivariate optimization problem that is often solved considering the throughput as the objective function and factors like transmission power, maximum transmission delay, allowable interference, QoS and fairness as the constrains. This optimization problem can be solved using classical optimization algorithms or using heuristic approaches.

In chapter 3, more OFDM, that has been widely accepted as the de-facto standard for $5 \mathrm{G}$ networks is used as the foundation ${ }^{1}$. Although different optimization strategies are possible, we focus on a modified GWF algorithm applied in general OFDM based cognitive radio

\footnotetext{
${ }^{1}$ Although Non Orthogonal Medium Access (NOMA) type algorithms are discussed for 5G networks, their performances are not yet proven and they require a significantly differential power transmission which may not be practical.
} 
systems considering per subchannel power constraint and individual peak power constraint aiming to maximize the throughput.

For this purpose, an efficient power and subcarrier allocation algorithm, named as IGPP is discussed. This algorithm is optimized in such a way to consider both individual subcarrier peak power constraint and per subchannel (group of subcarriers) power constraint. Simulation results are provided to illustrate the effectiveness of the algorithm. The proposed algorithm allows better utilization of the available power resources, thus maximizes the throughput than some other existing algorithms. The algorithm allocates more power to wireless channels that have less fading, hence saving power resources. Also a weight factor is considered for each channel to further fine tune the optimization.

Then in chapter 4, it is studied how efficiently D2D communications can be employed with a CR approach. Here, again an OFDM based cognitive cellular network with D2D communication has been modeled and analyzed using Lagrange formulation. The adaptive subcarrier allocation is optimized in terms of power, interference and transmission rate. The proposed algorithm based on GWF is tested to maximize the total downlink transmission rate of the D2D system under both power and interference constraints along with minimum rate requirements. Our developed scheme outperforms the existing schemes as revealed in the simulation results.

Chapter 5 investigates the EE power allocation problem of co-existing D2D and cellular users considering both underlay and overlay CR approaches. The underlay and overlay approaches are utilized in complementary fashion to support more practical scenarios. In both the (underlay and overlay) spectrum sharing schemes, the optimization problem is formulated as the maximization of the D2D users EE subject to the minimum rate requirement for both the D2D users and the cellular users. This leads to a nonconvex fractional optimization problem which is more complicated and computationally intractable. Alternatively, we utilize the proven GWF power allocation algorithm to solve this optimization problem. Contrast to the widely used Dinkelbach method for EE maximization, this algorithm can directly 
compute the optimal solution by applying the geometry-based approach, with exactness and low-degree polynomial complexity.

In a nutshell, we brought the awareness on the key issues in designing a reliable high performance cognitive D2D communication networks. The need for optimal RA is discussed and few algorithms proposed by the authors are used to perform an optimal RA for CRNs that co-exist with D2D communication.

\subsection{Future Research Directions}

In this section, the challenges in RA methods are discussed and the future research directions and opportunities are outlined.

\subsubsection{Spectrum Sensing Issues}

The existing RA schemes are developed under the assumption of having perfect spectrum sensing. However, this assumption may not be true and there may be errors in spectrum sensing which may direct to inefficient RA and interference with PUs. Therefore, discovery process of spectrum need to be carried out more carefully because it is influenced by three main issues:

- The hidden transmitter

- The exposed transmitter and

- The hidden receiver.

The hidden transmitter issue has been resolved by carrying out sensing operation at both transmitter and receiver ends, but there are still no satisfactory solutions for the latter issues. A CR user should be capable of discovering the existence of a neighboring primary receiver to resolve these issues. Hence, feasible solutions for this purpose are yet to be investigated. 
Furthermore, spectrum sensing performance is limited by hardware and physical constraints. For instance, SUs with a single transceiver cannot transmit and sense simultaneously. Moreover, users usually only observe a partial state of the network to limit sensing overhead. There is a fundamental trade-off between the undesired overhead and spectrum holes detection effectiveness: the more bands are sensed, the higher the number and quality of the available resource. Thus this problem needs to be further investigated to improve spectrum sensing effectiveness. Lastly, sensing errors due to false detection and miss detection in PU signal sensing are ignored in many cases in the literature. Accurate models that take both false alarm and mis-detection probabilities into account, need to be devised. Therefore, the simplified ON/OFF model for PU traffic may not be a proper choice for practical environment where PUs may be cellular or wireless sensors. To improve the probability of detection with less sensing errors, cooperative techniques have been proposed [143]. However, many aspects of cooperative sensing need to be still investigated for better solutions [144]. Future RA schemes have to incorporate more complex considerations such as the impact of correlation among sensing channels, imperfectness of reporting channels etc. to develop more efficient cooperative sensing schemes [145]. Furthermore, trade-off between sensing action and throughput as well as other applications such as multimedia application over CRNs needs to be further investigated.

\subsubsection{Channel Models}

The majority of the proposed RA algorithms have adopted a Rayleigh fading channel model. Few works considered Nakagami-m channel model [146]. However, it is suggested to test the existing algorithms and propose new algorithms for different channel models. Particularly, the environment where the CR entities communicate whether urban, sub-urban, rural, indoor, or outdoor will affect the wireless channel characteristics. For example, the deployment of a secondary network in a rural area with low building density increases the line-of-sight probability, and thus Rician fading channels would be more appropriate. Alter- 
ing the adopted channel model will definitely affect the analytical performance results and also could have an effect on the algorithms optimality. Furthermore, more efforts considering non-i.i.d channels is encouraged [147].

\subsubsection{Cross-layer Design Approaches and Security Issues}

In CRNs, superior interaction is needed between different layers of protocol stacks in order to attain desired goals and performance in terms of radio resource management, QoS provisioning, security and other network objectives. The idea behind the Cross-layer design [148] approach refers to devise the protocol stack by exploiting the information exchange and reliance between different protocol layers to acquire superior performance. But at the same time, it will also be susceptible to cross-layer attacks which may happen due to malevolent operations executed at one layer that could cause security violations at another layer [88]. CRNs inherently require greater interaction between different layers of the protocol stack. Therefore, cross-layer attacks and security related issues [149] including jamming attack and mitigation, selfish behavior in collaborative sensing and misbehavior in detection, physical layer and MAC layer in security, and the modeling and detection of insider attacks should be given special attention. A cross-layer security mechanism that incorporates recent advances on security threats/attacks and countermeasures, need to be investigated in RA design in order to permit a reliable and secure environment for CRNs [150]. Finally, the application of artificial intelligence (AI) techniques can be included to tackle security challenges for dynamic spectrum access [151].

\subsubsection{Spectrum Mobility Issues}

Spectrum mobility is also an essential issue in RA design aspect. Studies in the literature incorporate several design issues such as PU detection, handoff decision, target channel selection and spectrum handoff strategy [152]. Spectrum sensing speed and precision that greatly influence PU detection event, can be increased by cooperative sensing [153]. Handoff decision 
is another important issue which can cause harmful interference to PU. This can be greatly improved by using proper handoff algorithm e. g. fuzzy logic based algorithm [154]. Appropriate target channel selection approaches in the literature include having a backup channel, target channel availability prediction under partial sensing scheme and selection utilizing historical data. PU traffic modeling taking PU mobility [155] into account needs careful attention in order to intelligently select the target channel by SUs. Several prediction and estimation schemes [156], like hidden Markov models [157], neural networks [158], Bayesian inference [159] and, autoregressive model [160] can be adopted for better realization of PU activity. Proper spectrum handoff strategies need to be chosen that are adaptive in nature according to PU traffic. In order to minimize delay in handoff event, simultaneous data transmission in multi-channel CRNs need to be performed. Effective contention resolution for multiple SUs during handoff need to be carried out for successful handoff. Cross-layer approach for link maintenance is necessary to effectively address mobility issues in physical, MAC and network layers. Finally an integrated handoff management process [161] is necessary to improve network performance.

\subsubsection{Multi-hop Scenario}

Dynamic spectrum access in multi-hop domain incorporates several challenges in RA schemes. Several studies [162], [163] in the literature tried to bring up research issues regarding RA design for multi-hop CRNs which can be summarized as:

1. Control channel establishment and management scheme without a predefined dedicated control channel,

2. Transceiver synchronization,

3. CTS timeout and problems in decoding CTS,

4. Multi-channel hidden terminal problem, 
5. Hidden incumbent node problem,

6. Number of transceivers,

7. Coordination of spectrum sensing and accessing decision making,

8. Radio frequency heterogeneity,

9. Group communication, and

10. MAC layer authentication.

Some other attributes like the effect of channel state information in spectrum access, QoS guarantee, concern in fairness aspect, PU protection also need careful attention and further investigation in multi-hop environment.

\subsubsection{QoS Management in CR Environment}

Spectrum utilization and network capacity of CRNs can be increased by dynamic spectrum supervision. However, this imposes several challenges in QoS management [164]. Users can exploit available spectrum successfully captured by a CR but accurate information needs to be forwarded to the application to amend traffic features and user requirements. Once the available frequency bands are characterized, the most suitable spectrum bands can be selected by taking into account the spectrum characteristics and QoS criteria. However, spectrum characteristics can alter due to the dynamic nature of PU traffic and network parameters. Thus, appropriate spectrum decisions and necessary interactions among application streams, need to be performed to meet the QoS provisioning. Several QoS supportive RA schemes are reported in the literature. Most of the approaches assumed that PU traffic characteristics are known by SUs. However, this may not be true in general. Hence, PU traffic parameter estimation needs to be done often [165]. Such accurate estimation is necessary to guarantee QoS criteria in terms of disruption caused to the PUs. Overall, further 
investigations are needed to design QoS supportive RA schemes for CRN based on different criteria.

\subsubsection{Cognitive Heterogeneous Networks (HetNets)}

Cognitive heterogeneous networks (HetNets) are an attractive solutions for expanding network capacity to multiple spectrum access technologies, network structures and communication protocols. Some applications such as tactical applications in military communications [166], medical applications in CR-based wireless body area networks [167], are few interesting recent applications of cognitive HetNets. A thorough study of RA schemes with the capability of cognitive HetNets is still an open area of research. However, only a few works address the issues in a coexistent heterogeneous CRNs scenario [168]. Since the system characteristics of these cognitive HetNets, are different in several categories such as spectrum sensing aspects, PU detection ability, hardware capacity, fairness issue, all these categories need to be explored while designing MAC structure. A scenario of coexistent heterogeneous CRNs with collision-based PUs and fairness issue are discussed in [169]. Further research towards opportunistic $3 \mathrm{G} / 4 \mathrm{G} / 5 \mathrm{G}$ spectrum sharing involve precise evaluation of the scenario conditions, in terms of terminal confinement, link maintenance, and user preferences, and will also need to consider face side aspects such as security supervision and privacy preservation. The rising requirement of wireless data traffic and the scarceness of accessible radio spectrum, will extend 3GPP's LTE (Release 8) and LTE-Advanced (Release 10) to unlicensed bands (5 GHz). Thus dynamic spectrum management for LTE [170] and LTEAdvanced [171], [172] mobile communication network as a CR-ready technology, become challenging and provide an active area for research. 
Appendix 1

Acronyms 


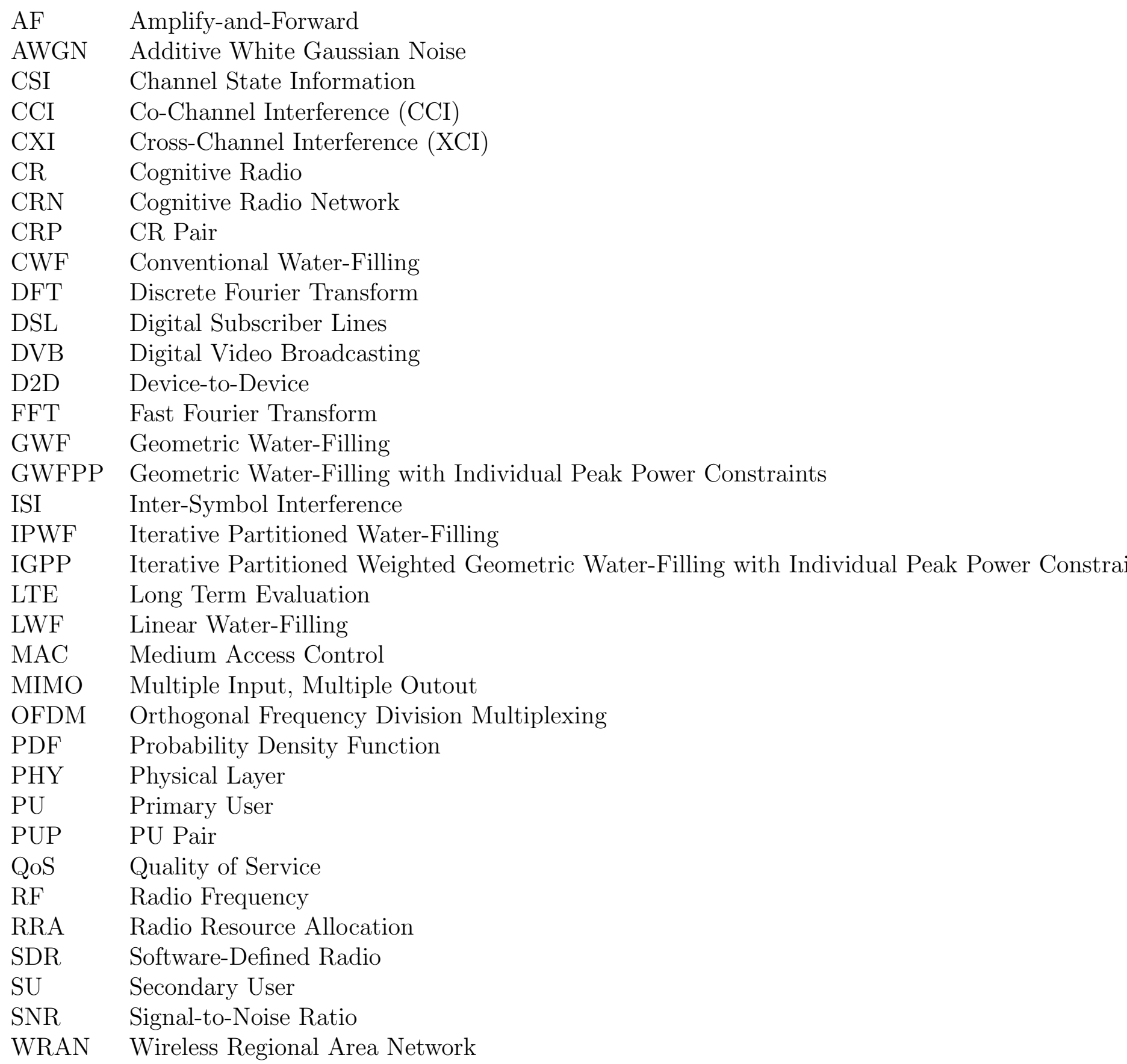




\section{Appendix 2}

\section{Derivation of $P_{m, n}^{*}$}

In order to find the solution of the optimization problem depicted in (4.9), Lagrange multipliers $(\alpha, \beta, \gamma, \delta, \psi)$ are used corresponding to the five constraints. The Lagrange can be formed as

$$
\begin{aligned}
& L(\mathbf{X}, \mathbf{T}, \alpha, \beta, \gamma, \delta, \psi)=\Delta B \sum_{m=1}^{M} \sum_{n=1}^{N} x_{m, n} \log \left(1+\frac{t_{m, n}}{x_{m, n}} \Gamma_{m, n}\right)-\sum_{m=1}^{M} \sum_{n \in \mathcal{N}_{m}} \alpha_{n}\left(\sum_{m=1}^{M} \sum_{n=1}^{N} t_{m, n}-P_{T}\right) \\
& -\sum_{m=1}^{M} \beta_{m}\left(\sum_{n=1}^{N} t_{m, n}-P_{m, \max }\right)-\sum_{m=1}^{M} \sum_{n \in \mathcal{N}_{m}} \gamma_{n}\left(t_{m, n}-\bar{P}_{m, n}\right)-\sum_{k=1}^{K} \delta_{k}\left(\sum_{m=1}^{M} \sum_{n=1}^{N} t_{m, n} I_{m, n}^{(k)}-I_{t h}\right) \\
& -\sum_{m=1}^{M} \psi_{m}\left(\Re_{m, \min }-\sum_{n=1}^{N} x_{m, n} \Re_{m, n}\right)
\end{aligned}
$$


The Lagrangian dual problem can be rewritten as follows:

$$
\begin{aligned}
& D(\alpha, \beta, \gamma, \delta, \psi)=\max _{\mathbf{X}, \mathbf{T}} \sum_{m=1}^{M} \sum_{n=1}^{N}\left\{\left(1+\psi_{m}\right) x_{m, n} \log \left(1+\frac{t_{m, n}}{x_{m, n}} \Gamma_{m, n}\right)-\left(\alpha_{n}+\beta_{m}+\gamma_{n}+\sum_{k=1}^{K} \delta_{k}\right.\right. \\
& \left.\left.I_{m, n}^{(k)}\right) t_{m, n}\right\}+\sum_{m=1}^{M} \sum_{n \in \mathcal{N}_{m}} \alpha_{n} P_{T}+\sum_{m=1}^{M} \beta_{m} P_{m, \max }+\sum_{m=1}^{M} \sum_{n \in \mathcal{N}_{m}} \gamma_{n} \bar{P}_{m, n}+\sum_{k=1}^{K} \delta_{k} I_{t h}-\sum_{m=1}^{M} \psi_{m} \Re_{m, m i n} \\
& =\max _{\mathbf{X}} \sum_{m=1}^{M} \sum_{n=1}^{N} \max _{\mathbf{P}}\left[x_{m, n}\left\{\left(1+\psi_{m}\right) \log \left(1+P_{m, n} \Gamma_{m, n}\right)-\left(\alpha_{n}+\beta_{m}+\gamma_{n}+\sum_{k=1}^{K} \delta_{m} I_{m, n}^{(k)}\right) P_{m, n}\right\}\right] \\
& +\sum_{m=1}^{M} \sum_{n \in \mathcal{N}_{m}} \alpha_{n} P_{T}+\sum_{m=1}^{M} \beta_{m} P_{m, m a x}+\sum_{m=1}^{M} \sum_{n \in \mathcal{N}_{m}} \gamma_{n} \bar{P}_{m, n}+\sum_{k=1}^{K} \delta_{k} I_{t h}-\sum_{m=1}^{M} \psi_{m} \Re_{m, m i n} \\
& =\max _{\mathbf{X}} \sum_{m=1}^{M} \sum_{n=1}^{N} \max _{\mathbf{P}}\left\{x_{m, n} \Psi\left(P_{m, n}\right)\right\}+\sum_{m=1}^{M} \sum_{n \in \mathcal{N}_{m}} \alpha_{n} P_{T}+\sum_{m=1}^{M} \beta_{m} P_{m, m a x}+\sum_{m=1}^{M} \sum_{n \in \mathcal{N}_{m}} \gamma_{n} \bar{P}_{m, n} \\
& +\sum_{k=1}^{K} \delta_{k} I_{t h}-\sum_{m=1}^{M} \psi_{m} \Re_{m, \min }
\end{aligned}
$$

with $\Psi\left(P_{m, n}\right)$ being defined as follows:

$$
\Psi\left(P_{m, n}\right)=\left(1+\psi_{m}\right) \log \left(1+P_{m, n} \Gamma_{m, n}\right)-\left(\alpha_{n}+\beta_{m}+\gamma_{n}+\sum_{k=1}^{K} \delta_{k} I_{m, n}^{(k)}\right) P_{m, n}
$$

To maximize (2.2) for any given $x_{m, n},(2.3)$ is differentiated with respect to $P_{m, n}$ and set the result to 0 . This yields

$$
P_{m, n}^{*}=\left[\frac{1+\psi_{m}}{\ln \left(\alpha_{n}+\beta_{m}+\gamma_{n}+\sum_{k=1}^{K} \delta_{k} I_{m, n}^{(k)}\right)}-\frac{1}{\Gamma_{m, n}}\right]^{+}
$$




\section{References}

[1] I. Akyildiz, W. Lee, M. Vuran, and S. Mohanty, "Next generation/ dynamic spectrum access/cognitive radio wireless networks: a survey," Elsevier Computer Networks, vol. 50, no. 13, pp. 2127 - 2159, Sep 2006.

[2] Federal Communications Commission, "Spectrum policy task force," Report of ET Docket, pp. $02-135$, Nov 2002.

[3] G. Taylor, C. Middleton, and X. Fernando, "A Question of Scarcity: Spectrum and Canada's Urban Core," E-ISSN: 2158-3897, 2016.

[4] J. Mitola, "Cognitive radio: an integrated agent architecture for software defined radio," PhD thesis, KTH Royal Institute of Technology, Stockholm, Sweden, 2000.

[5] S. Haykin, "Cognitive radio: brain-empowered wireless communications," IEEE Journal on Selected Areas in Communications (JSAC), vol. 23, no. 2, pp. 201 - 220, Feb 2005.

[6] S. Hijazi, B. Natarajan, M. Michelini, Z. Wu, and C. Nassar, "Flexible spectrum use and better coexistence at the physical layer of future wireless systems via a multicarrier platform," IEEE Wireless Comm., vol. 11, no. 2, pp. 8 -14, Apr 2004.

[7] A. S. Zahmati, X. Fernando, and A. Grami, "Steady-state Markov chain analysis for heterogeneous cognitive radio networks," IEEE Sarnoff Symposium, pp. 1 - 5, Apr 2010. 
[8] IEEE standard for information technology, telecommunications and information exchange between systems WRAN specific requirements - Part 22: cognitive wireless RAN Medium Access Control (MAC) and Physical Layer (PHY) specifications, IEEE 802.22a (Amendment to the IEEE Std-802.22-2011(TM)), ," https://standards . ieee.org/about/get/802/802.22.html, 2014.

[9] Standard for wireless LAN in TV white space, IEEE 802.11af, ," https://standards . ieee.org/news/2014/ieee802.11af, Feb 2014.

[10] IEEE 1900 Standards, ," http://grouper.ieee.org/groups/dyspan/.

[11] IEEE 802.19: TV white space coexistence methods, ," https://standards.ieee. org/about/get/802/802.19.html.

[12] ETSI, "Mobile broadband services in the 2,300 MHz 2,400 MHz frequency band under licensed shared access regime," 2013.

[13] M. Matinmikko, M. Palola, H. Saarnisaari, M. Heikkila, J. Prokkola, T. Kippola, T. Hanninen, M. Jokinen, and S. Yrjola, "Cognitive radio trial environment: first live authorized shared access-based spectrum-sharing demonstration," IEEE Vehiculat Technology Magazine, vol. 8, no. 3, pp. 30 - 37, Sep 2013.

[14] IEEE 802.15 Working Group, "Wireless medium access control (MAC) and physical layer (PHY) specifications for low-rate wireless personal area networks (WPANs)," http://www. ieee802.org/15/pub/TG4m.html, [Online; accessed Jun-2012].

[15] A. Asadi, Q. Wang, and V. Mancusoi, "A survey on device-to-device communication in cellular networks," vol. 16, no. 4, pp. 1801 - 1819, Apr. 2014.

[16] D. Feng, L. Lu, Y. Yuan-Wu, G. Y. Li, G. Feng, and S. Li, "Device-to-device communications underlaying cellular networks," IEEE Trans. on Communications, vol. 61, no. 8, pp. 3541 - 3551, Aug. 2013. 
[17] W. Alliance, "Wi-fi peer-to-peer (p2p) specification v1. 1," Wi-Fi Alliance Specification, vol. 1, pp. 1 - 159, 2010.

[18] S. Bluetooth, "Bluetooth specification version 1.1," http://www.bluetooth.com, 2001.

[19] P. Cheng, L. Deng, H. Yu, Y. Xu, and H. Wang, "Resource allocation for cognitive networks with D2D communication: An evolutionary approach," pp. 2671 - 2676, Apr. 2012.

[20] M. N. Tehrani, M. Uysal, and H. Yanikomeroglu, "Device-to-device communication in 5G cellular networks: challenges, solutions, and future directions," IEEE Communications Magazine, vol. 52, no. 5, pp. 86 - 92, May 2014.

[21] L. Y. Song, D. Niyato, Z. Han, and E. Hossain, "Game-theoretic resource allocation methods for device-to-device (D2D) communication," vol. 21, no. 3, pp. 136 - 144, 2014.

[22] A. Ali and W. Hamouda, "Advances on spectrum sensing for cognitive radio networks: theory and applications," IEEE Communications Surveys and Tutorials, vol. PP, no. 99, pp. 1 - 29, nov 2016.

[23] A. H. Sakr, H. Tabassum, E. Hossain, and D. I. Kim, "Cognitive spectrum access in device-to-device-enabled cellular networks," IEEE Communications Magazine, vol. 53, no. 7 , pp. 126 - 133, July 2015.

[24] W. Xiong, A. Mukherjee, and H. M. Kwon, "MIMO cognitive radio user selection with and without primary channel state information," IEEE Trans. Veh. Technol., vol. 65, no. 2, pp. 985 - 991, Feb. 2016. 
[25] E. Driouch and W. Ajib, "Downlink scheduling and resource allocation for cognitive radio MIMO networks," IEEE Trans. Veh. Technol., vol. 62, no. 8, pp. 3875 - 3885, Oct. 2013.

[26] S. Wang, F. Huang, and C. Wang, "Adaptive proportional fairness resource allocation for OFDM-based cognitive radio networks," Wireless Netw., vol. 19, no. 3, pp. 273 284, Apr. 2013.

[27] L. Lu, D. He, X. Yu, and G. Y. Li, "Energy-efficient resource allocation for cognitive radio networks," in Proc. IEEE Glob. Commun. Conf. (GLOBECOM), Atlanta, GA, USA, pp. 1026 - 1031, Dec. 2013.

[28] H. Guo and V. C. M. Leung, "Orthogonal transmissions for spectrum underlay MISO cognitive radio," IEEE Trans. Wireless Commun., vol. 11, no. 4, pp. 1266 - 1270, Apr. 2012.

[29] A. G. Marques, L. M. Lopez-Ramos, G. B. Giannakis, and J. Ramos, "Resource allocation for interweave and underlay CRs under probability-of-interference constraints," IEEE J. Sel. Areas Commun., vol. 30, no. 10, pp. 1922 - 1933, Nov. 2012.

[30] L. B. Le and E. Hossain, "Resource allocation for spectrum underlay in cognitive radio networks," IEEE Trans. Wireless Commun., vol. 7, no. 12, pp. 5306 - 5315, Dec. 2008.

[31] J. R. Gllego, M. Canales, and J. Ortn, "Distributed resource allocation in cognitive radio networks with a game learning approach to improve aggregate system capacity," Elsevier Ad Hoc Netw., vol. 10, no. 6, pp. 1076 - 1089, Aug. 2012.

[32] Y. Xu and X. Zhao, "Distributed power control for multiuser cognitive radio networks with quality of service and interference temperature constraints," Wireless Commun. Mobile Comput., vol. 15, no. 14, pp. 1773 - 1786, Oct. 2015. 
[33] S. Gong, P. Wang, and L. Duan, "Distributed power control with robust protection for PUs in cognitive radio networks," IEEE Trans. Wireless Commun., vol. 14, no. 6, pp. 3247 - 3258, June 2015.

[34] M. Rasti, M. Hasan, L. B. Le, and E. Hossain, "Distributed uplink power control for multi-cell cognitive radio networks," IEEE Trans. Commun., vol. 63, no. 3, pp. 628 642, Mar. 2015.

[35] J. Zhang, Z. Zhang, H. Luo, A. Huang, and R. Yin, "Uplink scheduling for cognitive radio cellular network with primary users QoS protection," in Proc. IEEE Wireless Commun. Netw. Conf. (WCNC), Sydney, NSW, Australia, pp. 1-5, Apr. 2010.

[36] R. Sumathi, M. Poornima, and M. Suganthi, "User aware mobility management in cognitive radio cellular network," in Proc. Int. Conf. Electron. Commun. Syst. (ICECS), Coimbatore, India, pp. 1 - 5, Feb. 2014.

[37] C. R. Stevenson, G. Chouinard, Z. Lei, W. Hu, S. J. Shellhammer, and W. Caldwell, "IEEE 802.22 The first cognitive radio wireless regional area network standard," IEEE Commun. Mag., vol. 47, no. 1, pp. 130 - 138, Jan. 2009.

[38] A. Dimogiorgi and W. Hamouda, "A proposed enhanced scheme for the dynamic frequency hopping performance in the IEEE 802.22 standard," Wireless Commun. Mobile Comput. J., vol. 16, no. 16, pp. 2714 - 2729, July 2016.

[39] M. E. Tanab, W. Hamouda, and Y. Fahmy, "On the distributed resource allocation of MIMO cognitive radio networks," in Proc. IEEE Glob. Commun. Conf. (GLOBECOM), San Diego, CA, USA, pp. 1 -6, Dec. 2015.

[40] R. Jurdak, C. V. Lopes, and P. Baldi, "A survey, classification and comparative analysis of medium access control protocols for ad hoc networks," IEEE Commun. Surveys Tuts., 12004. 
[41] I. F. Akyildiz, W.-Y. Lee, and K. R. Chowdhury, "CRAHNs Cognitive radio ad hoc networks," Ad Hoc Netw., vol. 7, no. 5, pp. 810 - 836, July 2009.

[42] S.-J. Kim and G. B. Giannakis, "Optimal resource allocation for MIMO ad hoc cognitive radio networks," IEEE Trans. Inf. Theory, vol. 57, no. 5, pp. 3117 - 3131, May 2011.

[43] I. F. Akyildiz, W.-Y. Lee, and K. R. Chowdhury, "Spectrum management in cognitive radio ad hoc networks," IEEE Netw., vol. 23, no. 4, pp. 6 - 12, Aug. 2009.

[44] N. Kokash, "An introduction to heuristic algorithms," Department of Informatics and Telecommunications, pp. $1-8,2005$.

[45] E. A. Silver, "An overview of heuristic solution methods," Journal of the operational research society, vol. 55, no. 9, pp. 936 - 956, May 2017.

[46] M. E. Tanab, Y. Fahmy, and M. M. Khairy, "Opportunistic splitting algorithm for underlay cognitive radio networks," in Proc. IEEE Sixth Int. Conf. on Ubiquitous and Future Networks (ICUFN), pp. 9 - 14, July 2014.

[47] B. Wang, D. Zhao, and J. Cai, "Joint connection admission control and packet scheduling in a cognitive radio network with spectrum underlay," IEEE Transactions on Wireless Communications, vol. 10, no. 11, pp. 3852 - 3863, Nov. 2011.

[48] E. Driouch and W. Ajib, "Downlink scheduling and resource allocation for cognitive radio MIMO networks," IEEE Transactions on Vehicular Technology, vol. 62, no. 8, pp. 3875 - 3885, Oct. 2013.

[49] S. Wang, F. Huang, and C. Wang, "Adaptive proportional fairness resource allocation for OFDM-based cognitive radio networks," Wireless Networks, vol. 19, no. 3, pp. 273 - 284, Apr. 2013. 
[50] S. Boyd and L. Vandenberghe, ," Convex Optimization., Cambridge, U.K., Cambridge University, 2004.

[51] L. Fu, M. Johansson, and M. Bengtsson, "Energy efficient transmissions in cognitive MIMO Systems with multiple data streams," IEEE Transactions on Vehicular Technology, vol. 14, no. 9, pp. 5171 - 5184, Sept. 2015.

[52] R. Kannan and C. L. Monma, ," On the computational complexity of integer programming problems, Springer Berlin Heidelberg, 1978.

[53] E. Z. Tragos, S. Zeadally, A. G. Fragkiadakis, and V. A. Siris, "Spectrum assignment in cognitive radio networks: A comprehensive survey," IEEE Communications Surveys and Tutorials, vol. 15, no. 3, pp. 1108 - 1135, Third quarter, 2013.

[54] E. Driouch, W. Ajib, and A. B. Dhaou, "A greedy spectrum sharing algorithm for cognitive radio networks,", in Proc. IEEE Int. Conf. on Computing, Networking and Communications (ICNC), pp. 1010 - 1014, Jan. 2012.

[55] Y.-J. Chang, Z. Tao, J. Zhang, and C-C. J. Kuo, "A graph-based approach to multicell OFDMA downlink resource allocation," in Proc. IEEE Global Telecommunications Conference (GLOBECOM), pp. 1 - 6, Dec. 2008.

[56] Y. Liu and M. Tao, "Optimal channel and relay assignment in OFDM-based multirelay multi-pair two-way communication networks," IEEE Transactions on Communications, vol. 60, no. 2, pp. 317 - 321, Feb. 2012.

[57] R. J. Wilson, ," Introduction to Graph Theory, 4th ed. Prentice Hall, 1996.

[58] L. Lu, D. He, X. Yu, and G. Y. Li, "Energy-efficient resource allocation for cognitive radio networks," in Proc. IEEE Global Telecommunications Conference (GLOBECOM), pp. 1026 -1031, Dec. 2013. 
[59] L. Lu, D. He, X. Yu, and G. Y. Li, "Graph-based robust resource allocation for cognitive radio networks,", in Proc. IEEE Int. Conf. on Acoustics, Speech and Signal Processing (ICASSP), pp. 7298 - 7302, May 2014.

[60] W. Zhou, T. Jing, W. Cheng, T. Chen, and Y. Huo, "Combinatorial auction based channel allocation in cognitive radio networks," in Proc. IEEE Int. Conf. on Cognitive Radio Oriented Wireless Networks (CROWNCOM), pp. 135 - 140, July 2013.

[61] L. Chen, L. Huang, H. Xu, H. Deng, and Z. Sun, "Optimal channel assignment schemes in underlay CRNs with multi-PU and multi-SU transmission pairs," Wireless Algorithms, Systems, and Applications, vol. 9204, pp. 29 - 39, Aug. 2015.

[62] C. Peng, H. Zheng, and B. Y. Zhao, "Utilization and fairness in spectrum assignment for opportunistic spectrum access," Mobile Networks and Applications, vol. 11, no. 4, pp. 555 - 576, Aug. 2006.

[63] K. Akkarajitsakul, E. Hossain, D. Niyato, and D. I. Kim, "Game theoretic approaches for multiple access in wireless networks: A survey," IEEE Communications Surveys and Tutorials, vol. 13, no. 3, pp. 372 - 395, Third quarter, 2011.

[64] M. Felegyhazi and J.-P. Hubaux, "Game theory in wireless networks: A tutorial,", IEEE Communications Surveys and Tutorials, No. LCA-REPORT-2006-002, 2006.

[65] B. Wang, Y. Wu, and K.J. R. Liu, "Game theory for cognitive radio networks: An overview," Elsevier Computer networks, vol. 54, no. 14, pp. 2537 - 2561, Oct. 2010.

[66] O. B. Abdulghfoor, M. Ismail, and R. Nordin, "Application of game theory to underlay ad-hoc cognitive radio networks: An overview," in Proc. IEEE Int. Conf. on Space Science and Communication (IconSpace). 
[67] A. De Domenico, E. C. Strinati, and M. Di Benedetto, "A survey on MAC strategies for cognitive radio networks," IEEE Communications Surveys and Tutorials, vol. 14, no. 1, pp. $21-44$, First quarter, 2012.

[68] H.-S. Lang, S.-C. Lin, , and W.-H. Fang, "Subcarrier pairing and power allocation with interference management in cognitive relay networks based on genetic algorithms," IEEE Transactions on Vehicular Technology, vol. 65, no. 9, pp. 7051 - 7063, Sept. 2016.

[69] Y. Jiao and I. Joe, "Energy-efficient resource allocation for heterogeneous cognitive radio network based on two-tier crossover genetic algorithm," Journal of Communications and Networks, 2016.

[70] Y. E. Morabit, F. Mrabti, and E. H. Abarkan, "Spectrum allocation using genetic algorithm in cognitive radio networks," Third Int. Workshop on RFID and Adaptive Wireless Sensor Networks (RAWSN).

[71] B. Atakan and O. B. Akan, "Biologically-inspired spectrum sharing in cognitive radio networks," in Proc. IEEE Wireless Communications and Networking Conference, (WCNC), pp. 43 - 48, Mar. 2007.

[72] S. Yang, J. Wang, Y. Han, and Q. Zhao, "Dynamic spectrum allocation algorithm based on fairness for smart grid communication networks," in Proc. IEEE Int. Conf. of Chinese Control Conference (CCC), pp. 6873 - 6877, July 2016.

[73] S. B. Behera and D. D. Seth, "Resource allocation for cognitive radio network using particle swarm optimization," in Proc. IEEE Int. Conf. on Electronics and Communication Systems (ICECS), pp. 665 - 667, Feb. 2015.

[74] B. Chen, M. Zhao, M. Lei, and L. Zhang, "An optimal spectrum sharing method for mimo cognitive radio networks," in Proc. IEEE Int. Symp. on Personal, Indoor, and Mobile Radio Communications (PIMRC), pp. 1007 - 1011, Sept. 2015. 
[75] P. Tiwari and S. Saha, "Co-channel interference constrained spectrum allocation with simultaneous power and network capacity optimization using PSO in cognitive radio network," in Proc. IEEE Int. Conf. on Advanced Networks and Telecommuncations Systems (ANTS), pp. 1 - 3, Dec. 2015.

[76] D. P. Palomar and J. R. Fonollosa, "Practical algorithms for a family of water-filling solutions," vol. 53, no. 2, pp. 686 - 695, Feb. 2005.

[77] W. Yu and J. M. Cioffi, "On constant power water-filling," in Proc. IEEE Int. Conference on Communications, vol. 6, pp. 1665 - 1669, Jun 2001.

[78] P. He, L. Zhao, S. Zhou, and Z. Niu, "Water-Filling: A geometric approach and its application to solve generalized radio resource allocation problems," IEEE transactions on Wireless Communications, pp. 3637 - 3646, July 2013.

[79] E. Hosseini and A. Falahati, "Power allocation grouping scheme considering constraints in two separate stages for OFDM-based cognitive radio system," in Proc. IEEE Int. Conf. Electrical Information and Communication Technology (EICT), pp. $1-6$, Feb 2014.

[80] S. Chakraborty, P. Dhanuka, A. Kumar, and S. P. Maity, "Subcarrier and power allocation schemes for multiuser OFDM-based cognitive radio systems," in Proc. IEEE National Conference on Communications (NCC), pp. 1-5, Feb 2013.

[81] Q. Qi, A. Minturn, and Y. Yang, "An efficient water-filling algorithm for power allocation in OFDM-based cognitive radio systems," in Proc. IEEE International Conference on Systems and Informatics (ICSAI), pp. 2069 - 2073, May 2012.

[82] W. Jian, Y. Longxiang, and L. Xu, "Resource allocation based on linear waterfilling algorithm in CR systems," in Proc. IEEE Wireless Communications, Networking and Mobile Computing (WiCOM), pp. 1 -4, Sept. 2011. 
[83] X. Zhou, B. Wu, P.-H. Ho, and X. Ling, "An efficient power allocation algorithm for OFDM based underlay cognitive radio networks," in Proc. IEEE Global Telecommunications Conference (GLOBECOM), pp. 1 - 5, Dec 2011.

[84] C.-H. Chen and C.-L. Wang, "An efficient power allocation algorithm for multiuser OFDM-based cognitive radio systems," in Proc. IEEE Wireless Communications and Networking Conference (WCNC), pp. 1-6, April 2010.

[85] Y. Zhang and C. Leung, "An efficient power-loading scheme for OFDM-based cognitive radio systems," IEEE Transactions on Vehicular Technology, vol. 59, pp. 1858 - 1864, May 2010.

[86] G. Bansal, O. Duval, and F. Gagnong, "Joint overlay and underlay power allocation scheme for OFDM-based cognitive radio systems," in Proc. IEEE Wireless Communications and Networking Conference (WCNC), pp. 1 - 5, May 2010.

[87] Z. Tang, G. Wei, and Y. Zhu, "Weighted sum rate maximization for OFDM-based cognitive radio systems," Telecommun Syst, vol. 42, pp. 77 - 84, Oct. 2009.

[88] P. Wang, M. Zhao, L. Xiao, S. Zhou, and J. Wang, "Power allocation in OFDMbased cognitive radio systems," in Proc. IEEE Global Telecommunications Conference (GLOBECOM), pp. 4061 - 4065, Nov 2007.

[89] Q. Zhao and B.M.Sadler, "A survey of dynamic spectrum access: signal processing, networking, and regulatory policy," IEEE Signal Processing Magazine, vol. 55, no. 5, pp. $2294-2309$, May 2007.

[90] D. Tse and P. Viswanath, Fundamentals of Wireless Communications, Cambridge University Press, May.

[91] G. I. Tsiropoulos, O. A. Dobre, M. H. Ahmed, and K. E. Baddour, "Radio resource allocation techniques for efficient spectrum access in cognitive radio networks," IEEE 
Communications Surveys and Tutorials, vol. 18, no. 1, pp. 824 - 847, First quarter, 2016.

[92] X. Li, N. Zhao, Y. Sun, and F. R. Yu, "Interference alignment based on antenna selection with imperfect channel state information in cognitive radio networks," IEEE Trans. Vehicular Tech., vol. 64, no. 7, pp. 5497 - 5511, July 2016.

[93] N. Zhao, F. R. Yu, H. Sun, and M. Li, "Adaptive power allocation schemes for spectrum sharing in interference-alignment-based cognitive radio networks," IEEE Trans. Vehicular Tech., vol. 65, no. 5, pp. 3700 - 3714, May 2016.

[94] H. Xie, B. Wang, F. Gao, and S. Jin, "A full-space spectrum-sharing strategy for massive MIMO cognitive radio," IEEE Journal on Selected Areas in Communications, vol. 34, no. 10, pp. 2537 - 2549, Oct. 2016.

[95] E. Bedeer, O. A. Dobre, M. H. Ahmed, and K. E. Baddour, "Rate - interference tradeoff in OFDM-based cognitive radio systems," IEEE Trans. on Wireless Communications, vol. 64 , no. 9, pp. $4292-4298$, Oct. 2015.

[96] E. Bedeer, O. Amin, O. A. Dobre, M. H. Ahmed, and K. E. Baddour, "Energy-efficient power loading for OFDM-based cognitive radio systems with channel uncertainties," IEEE Trans. Vehicular Tech., vol. 64, no. 6, pp. 2672 - 2677, July 2015.

[97] M. El-Absi, M. Shaat, F. Bader, and T. Kaiser, "Interference alignment with frequencyclustering for efficient resource allocation in cognitive radio networks," IEEE Trans. on Wireless Communications, vol. 14, no. 12, pp. 7070 - 7082, Dec. 2015.

[98] E. Bedeer, O. A. Dobre, M. H. Ahmed, and K. E. Baddour, "A multiobjective optimization approach for optimal link adaptation of OFDM-based cognitive radio systems with imperfect spectrum sensing," IEEE Trans. on Wireless Communications, vol. 13, no. 4, pp. 2339 - 2351, Apr. 2014. 
[99] E. Bedeer, O. A. Dobre, M. H. Ahmed, and K. E. Baddour, "Joint optimization of bit and power loading for multicarrier systems," IEEE Wireless Communications Letters, vol. 2, no. 4, pp. 447 - 450, June 2013.

[100] J. Liu, S. Zhang, N. Kato, H. Ujikawa, and K. Suzuki, "Device-to-device communications for enhancing quality of experience in software defined multi-tier LTE-A networks," vol. 29, no. 4, pp. 46 - 52, July 2015.

[101] J. Liu, N. Kato, J. Ma, and N. Kadowaki, "Device-to-device communication in LTEAdvanced networks: A survey," vol. 17, no. 4, pp. 1923 - 1940, Dec. 2015.

[102] R. Yin, C. Zhong, G. Yu, Z. Zhang, K. K. Wong, and X. Chen, "Joint spectrum and power allocation for D2D communications underlaying cellular networks," IEEE Trans. Vehicular Tech., vol. 65, no. 4, pp. 2182 -2195, Apr. 2016.

[103] C. Xu, L. Y. Song, Z. Han, Q. Zhao, X. Wang, and B. Jiao, "Efficiency resource allocation for device-to-device underlay communication systems: A reverse iterative combinatorial auction based approach," vol. 31, no. 9, pp. 348 - 358, Sept. 2013.

[104] H. H. Esmat, M. M. Elmesalawy, and I. I. Ibrahim, "Adaptive resource sharing algorithm for device-to-device communications underlaying cellular networks," vol. 20, no. 3, pp. 530 - 533, Mar. 2016.

[105] W. Zhao and S. Wang, "Resource sharing scheme for device-to-device communication underlaying cellular networks," vol. 63, no. 12, pp. 4838 - 4848, Dec. 2015.

[106] D. Zhu, J. Wang, A. L. Swindlehurst, and C. Zhao, "Downlink resource reuse for deviceto-device communications underlaying cellular networks," IEEE Signal Processing Letters, vol. 21, no. 5, pp. 531 - 534, May 2014. 
[107] W. Liu, Y. Yang, T. Peng, and W. Wang, "Optimal resource allocation scheme for satisfying the data rate requirement in hybrid network of D2D-cellular," Journal of computers, vol. 9, no. 5, pp. 1218 - 1225, May 2014.

[108] Y. Jiang, Q. Liu, F. Zheng, X. Gao, and X. You, "Energy-efficient joint resource allocation and power control for D2D communications," IEEE Trans. Vehicular Tech., vol. 65, no. 8, pp. 6119 - 6127, Aug. 2016.

[109] X. Chen, R. Q. Hu, J. Jeon, and G. Wu, "Energy efficient resource allocation for D2D communication underlaying cellular networks," pp. 2943 - 2948, June 2015.

[110] M. G. Khoshkholgh, Y. Zhang, K.-C. Chen, K. G. Shin, and S. Gjessing, "Connectivity of cognitive device-to-device communications underlying cellular networks," IEEE Journal on Selected Areas in Communications, vol. 33, no. 1, pp. 81 - 99, Jan. 2015.

[111] A. H. Sakr and E. Hossain, "Cognitive and energy harvesting-based D2D communication in cellular networks: stochastic geometry modeling and analysis," IEEE Trans. on Communications, vol. 63, no. 5, pp. 1867 - 1880, May 2015.

[112] X. Wu, Y. Chen, X. Yuan, and M. E. Mkiramweni, "Joint resource allocation and power control for cellular and device-to-device multicast based on cognitive radio," IET Communications, vol. 8, no. 16, pp. 2805 - 2813, Nov. 2014.

[113] L. Wang, H. Jin, X. Ji, Y. Li, and M. Peng, "Power allocation optimization for D2D communication underlaying cognitive full duplex relay networks," in proc. IEEE Conf. on Wireless Communications, Networking and Mobile Computing (WiCOM), pp. 1 6, Apr. 2016.

[114] L. Wang, H. Jin, X. Ji, Y. Li, and M. Peng, "Power allocation for cognitive D2D communication assisted by two-way relaying," IEEE Sym. on Microwave, Antenna, Propagation and EMC Technologies for Wireless Communications (MAPE), pp. 1 - 6, Oct. 2013. 
[115] T. Weiss, J. Hillenbrand, A. Krohn, and F. K. Jondral, "Mutual interference in OFDMbased spectrum pooling systems," in Proc. IEEE Vehicular Tech. Conf. (VTC 2004Spring), vol. 4, pp. 1873 - 1877, May 2004.

[116] W. Yu and R. Lui, "Dual methods for nonconvex spectrum optimization of multicarrier systems," IEEE transactions on Communications, vol. 54, no. 7, pp. 1310 - 1322, July 2006.

[117] G. Bansal, M. J. Hossain, and V. K. Bhargava, "Optimal and suboptimal power allocation schemes for OFDM-based cognitive radio systems," vol. 7, no. 11, pp. 4710 - 4718, Nov. 2008.

[118] S. Chakraborty, P. Dhanuka, A. Kumar, and S. P. Maity, "Subcarrier and power allocation schemes for multiuser OFDM-based cognitive radio systems," in Proc. IEEE National Conf. on Communications (NCC), pp. 1-5, Feb. 2013.

[119] K. Illanko, M. Naeem, A. Anpalagan, and D. Androutsos, "Energy-efficient frequency and power allocation for cognitive radios in television systems," IEEE Systems Journal, vol. 10, no. 1, pp. 313 - 324, mar 2016.

[120] S. Wang, W. Shi, and C. Wang, "Energy-efficient resource management in OFDMbased cognitive radio networks under channel uncertainty," IEEE Trans. Commun., vol. 63, no. 9, pp. 3092-3102, Sep 2015.

[121] A. Alabbasi, Z. Rezki, and B. Shihada, "Energy efficient resource allocation for cognitive radios: A generalized sensing analysis," IEEE Trans. Wireless Commun., vol. 14, no. 5 , pp. $2455-2469$, may 2015.

[122] D. D. Penda, L. Fu, and M. Johansson, "Energy efficient D2D communications in dynamic TDD systems," IEEE Trans. Commun., vol. 65, no. 3, pp. 1260 - 1273, Mar 2017. 
[123] K. Yang, S. Martin, C. Xing, J. Wu, and R. Fan, "Energy-efficient power control for device-to-device communications," IEEE Journal on Select. Areas in Commun., vol. 34, no. 12, pp. 3208 - 3220, Dec 2016.

[124] T. D. Hoang, L. B. Le, and T. Le-Ngoc, "Energy-efficient resource allocation for D2D communications in cellular networks," IEEE Trans. Vehi. Tech., vol. 65, no. 9, pp. $6972-6986$, Sep 2016.

[125] Y. Jiang, Q. Liu, F. Zheng, X. Gao, and X. You, "Energy-efficient joint resource allocation and power control for D2D communications," IEEE Trans. Vehi. Tech., vol. 65, no. 8, pp. 6119 - 6127, Aug 2016.

[126] L. Wei, R. Q. Hu, Y. Qian, and G. Wu, "Energy-efficiency and spectrum efficiency of multihop device-to-device communications underlaying cellular networks," IEEE Trans. Vehi. Tech., vol. 65, no. 1, pp. 367 - 380, Jan 2016.

[127] F. Wang, C. Xu, L. Song, and Z. Han, "Energy-efficient resource allocation for deviceto-device underlay communication," IEEE Trans. Wireless Commun., vol. 14, no. 4, pp. 2082 - 2092, Apr 2015.

[128] A. Zappone, B. Matthiesen, and E. A. Jorswieck, "Energy efficiency in MIMO underlay and overlay device-to-device communications and cognitive radio systems," IEEE Trans. on Signal Process., vol. 65, no. 4, pp. 1026 - 1041, Feb. 2017.

[129] Z. Zhou, G. Ma, C. Xu, Z. Chang, and T. Ristaniemi, "Energy-efficient resource allocation in cognitive D2D communications: A game-theoretical and matching approach," IEEE Int. Conf. on Commun. (ICC), pp. 1 - 6, May 2016.

[130] A. Goldsmith, S. A. Jafar, I. Maric, and S. Srinivasa, "Breaking spectrum gridlock with cognitive radios: An information theoretic perspective," in Proc. of IEEE, vol. 97, no. 5, pp. 894 - 914, May 2009. 
[131] Q. Zhao and B.M. Sadler, "A survey of dynamic spectrum access," IEEE Sig. Proc. Mag., vol. 24, no. 3, pp. 79 - 89, May 2007.

[132] I.F. Akyildiz, W.Y. Lee, and K.R. Chowdury, "CRAHNs: Cognitive Radio Ad Hoc Networks," Journal of Ad Hoc Networks, vol. 7, no. 5, pp. 810 - 836, Jul 2009.

[133] P. Phunchongharn, E. Hossain, and D. I. Kim, "Resource allocation for device-to-device communications underlaying LTE-advanced networks," IEEE Wireless Commun., vol. 20, no. 4, pp. 91 - 100, Aug 2013.

[134] A. Alsharoa, H. Ghazzai, E. Yaacoub, M.-S. Alouini, and A. E. Kamal, "Joint bandwidth and power allocation for MIMO two-way relays-assisted overlay cognitive radio systems," IEEE Trans. Cognitive Commun. Netw., vol. 1, no. 4, pp. 383 - 393, Dec 2015.

[135] Y. Pei and Y.-C. Liang, "Resource allocation for device-to-device communications overlaying two-way cellular networks," IEEE Trans. Wireless Commun., vol. 12, no. 7, pp. 3611 - 3621, Jul 2013.

[136] R. Blasco-Serrano, R. Thobaben J. Lv, E. A. Jorswieck, and M. Skoglund, "Multiantenna transmission for underlay and overlay cognitive radio with explicit message learning phase," EURASIP J. Wireless Commun. Netw., Dec 2013, doi = 10.1186/1687-1499-2013-195.

[137] K. Doppler, C. B. Ribeiro, and J. Kneckt, "Advances in D2D communications: Energy efficient Service and Device Discovery Radio," in the proc. IEEE Int. Conf. on Wireless Commu., Vehi. Tech., Info. Theory and Aerospace Elec. Syst. Tech. (Wireless VITAE), pp. 1 - 6, Mar 2011.

[138] S. Berger, M. Kuhn, A. Wittneben, T. Unger, and A. Klein, "Recent advances in amplify-and-forward two-hop relaying," IEEE Commun. Mag., vol. 47, no. 7, pp. 50 56, Jul 2009. 
[139] M. Iwamura, H. Takahashi, and S. Nagata, "Relay technology in LTE advanced," NTT DOCOMO Tech. J., vol. 18, no. 2, pp. 31 - 36, Jul 2010.

[140] W. Dinkelbach, "On nonlinear fractional programming," Management Science, vol. 13, no. 7 , pp. $492-498$, mar 1967.

[141] P. He, S. Zhang, L. Zhao, and X. Shen, "Multi-channel power allocation for maximizing energy efficiency in wireless networks," IEEE transactions on Vehicular Technology, accepted for publication 2017.

[142] P. He and L. Zhao, "Solving a class of sum power minimization problems using generalized water-filling," IEEE transactions on Wireless Communications, vol. 14, no. 12, pp. 6792 - 6804, July 2015.

[143] T. Yucek and H. Arslan, "A survey of spectrum sensing algorithms for cognitive radio applications," IEEE Communications Surveys and Tutorials, vol. 11, no. 1, pp. 116 130, Mar 2009.

[144] S. Hussain and X. Fernando, "Closed-form analysis of relay-based cognitive radio networks over nakagami-m fading channels," IEEE Transactions on Vehicular Technology, vol. 63, no. 02, pp. 1193 - 1203, March 2014.

[145] S. Hussain and X. Fernando, "Performance analysis of relay-based cooperative spectrum sensing in cognitive radio networks over non-identical nakagami-m channels," IEEE Transactions on Communications, vol. 62, no. 8, pp. 2733 - 2746, Aug 2014.

[146] Y. Wang, P. Ren, Q. Du, and Z. Su, "Resource allocation and access strategy selection for QoS provisioning in cognitive networks," in Proc. IEEE Int. Conf. on Communcations (ICC), pp. 4637 - 4641, June 2012. 
[147] Y. Li and A. Nosratinia, "Hybrid opportunistic scheduling in cognitive radio networks," IEEE Transactions on Wireless Communications, vol. 11, no. 1, pp. 328 - 337, jan 2012.

[148] V. Srivastava and M. Motani, "Cross-layer design: a survey and the road ahead," IEEE Communication Magazine, vol. 43, no. 12, pp. 112 - 119, Dec 2005.

[149] K. Ren, H. Zhu, Z. Han, and R. Poovendran, "Security in cognitive radio networks," in Proc. IEEE Network, vol. 27, no. 3, pp. 2 - 3, May 2013.

[150] R. K. Sharma and D. B. Rawat, "Advances on security threats and countermeasures for cognitive radio networks: A survey," IEEE Communication Surveys and Tutorials, vol. 17, no. 2, pp. 1023 - 1043, May 2015.

[151] A. He, K. K. Bae, T. R. Newman, J. Gaeddert, K. Kim, R. Menon, L. Morales-Tirado, J. J. Neel, Y. Zhao, J. H. Reed, , and W. H. Tranter, "A survey of artificial intelligence for cognitive radios," IEEE Trans. Veh. Technol., vol. 59, no. 4, pp. 1578 - 1592, May 2010.

[152] I. Christian, S. Moh, I. Chung, and J. Lee, "Spectrum mobility in cognitive radio networks," IEEE Communication Magazine, vol. 50, no. 6, pp. 114 - 121, Jun 2012.

[153] X. Liu and Z. Ding, "ESCAPE: A channel evacuation protocol for spectrum-agile networks," in Proc. IEEE Int. Symposium New Frontiers in Dynamic Spectrum Access Networks (DySPAN), pp. 292 - 302, Apr 2007.

[154] L. Giupponi and A. I. Prez-Neira, "Fuzzy-based spectrum handoff in cognitive radio networks," in Proc. IEEE Int. Conf. Cognitive Radio Oriented Wireless Networks and Communications (CrownCom), pp. 1 - 6, May 2008. 
[155] I. Butun, A. C. Talay, D. T. Altilar, M. Khalid, and R. Sankar, "Impact of mobility prediction on the performance of cognitive radio networks," in Proc. IEEE Wireless Telecommunications Symposium (WTS), pp. 1-5, Apr 2010.

[156] X. Xing, T. Jing, W. Cheng, Y. Huo, and X. Cheng, "Spectrum prediction in cognitive radio networks," IEEE Wireless Communication, vol. 20, no. 2, pp. 90 - 96, Jun 2013.

[157] I. A. Akbar and W. H. Tranter, "Dynamic spectrum allocation in cognitive radio using hidden Markov models: poisson distributed case," in Proc. IEEE Southeastcon, pp. 196 - 201, Apr 2007.

[158] V. K. Tumuluru, P. Wang, and D. Niyato, "A neural network based spectrum prediction scheme for cognitive radio," in Proc. ICC, Cape Town, pp. 1-5, May 2010.

[159] X. Xing, T. Jing, Y. Huo, H. Li, and X. Cheng, "Channel quality prediction based on bayesian inference in cognitive radio networks," in Proc. IEEE INFOCOM, Turin, pp. 1465 - 1473, Apr 2013.

[160] Z. Wen, T. Luo, W. Xiang, S. Majhi, and Y. Ma, "Autoregressive spectrum hole prediction model for cognitive radio systems," in Proc. IEEE Int. Conf. on Communications Workshops, pp. 154 - 157, May 2008.

[161] S. Nejatian, S. K. Syed-Yusof, N. M. Abdul Latiff, and V. Asadpour, "Integrated handoff management in cognitive radio mobile ad hoc networks," in Proc. IEEE Int. Symp. on Personal Indoor and Mobile Radio Communications (PIMRC), pp. 2887 2892, Sep 2013.

[162] D. Gzpek and F. Alagz, An opportunistic pervasive networking paradigm: multi-hop cognitive radio networks, in pervasive computing and networking (eds M. S. Obaidat, M. Denko and I. Woungang), John Wiley and Sons, Ltd, Chichester, UK., Jun 2011, DOI:10.1002/9781119970422.ch7. 
[163] K. M. F. Rabbi, D. B. Rawat, M. A. Ahad, and T. Amin, "Analysis of multi-hop opportunistic communications in cognitive radio network," in Proc. IEEE SoutheastCon, Fort Lauderdale, FL, pp. 1-8, Apr 2015.

[164] M. Kartheek and V. Sharma, "Providing QoS in a cognitive radio network," in proc. of IEEE Int. Conf. on Communication Systems and Networks (COMSNETS), pp. 1 9, Jan 2012.

[165] A. Sahoo and M. Souryal, "Implementation of an opportunistic spectrum access system with disruption QoS provisioning and PU traffic parameter estimation," in proc. of IEEE Int. Conf. Wireless Communications and Networking Conference (WCNC), pp. 1084 - 1089, Mar 2015.

[166] M. Suojanen and J. Nurmi, "Tactical Applications of Heterogeneous Ad Hoc Networks Cognitive Radios, Wireless Sensor Networks and COTS in Networked Mobile Operations," In the proc. of Int. Conf. on Advances in Cognitive Radio (COCORA), pp. 1 -5 , Feb 2014.

[167] R. Chvez-Santiago and I. Balasingham, "Cognitive Radio for Medical Wireless Body Area Networks," in the proc. of IEEE Int. Workshop on Computer Aided Modeling and Design of Communication Links and Networks (CAMAD), pp. 148 - 152, Jun 2011.

[168] G. A. Shah, V. C. Gungor, and O. K. Akan, "A cross-layer design for QoS support in cognitive radio sensor networks for smart grid applications," in Proc. IEEE Int. Conf. on Communcations (ICC), pp. 1378 - 1382, June 2012.

[169] Y-C. Cheng, E. H. Wu, and G-H. Chen, "A Decentralized MAC Protocol for Unfairness Problems in Coexistent Heterogeneous Cognitive Radio Networks Scenarios With Collision-Based Primary Users," IEEE Systems Journal, vol. PP, no. 99, pp. 1 - 12, Jun 2015. 
[170] J. Naranjo, I. Viering, and K. Friederichs, "A cognitive radio based dynamic spectrum access scheme for lte heterogeneous networks," in proc. of IEEE Wireless Telecommunications Symposium (WTS), pp. 1 - 7, Apr 2012.

[171] J. Deaton, R. Irwin, and L. DaSilva, "The effects of a dynamic spectrum access overlay in lte-advanced networks," in Proc. IEEE Symposium on New Frontiers in Dynamic Spectrum Access Networks (DySPAN), pp. 488 - 497, May 2011.

[172] J. D. Naranjo, G. Bauch, A. B. Saleh, I. Viering, and R. Halfmann, "A Dynamic Spectrum Access Scheme for an LTE-Advanced HetNet with Carrier Aggregation," in Proc. Int. ITG Conf. on Systems, Communication and Coding (SCC), pp. $1-6$, Jan 2013. 
REFERENCES 